\title{
Asymmetric Diels-Alder Reactions of 2-Pyrones with a Bifunctional Organic Catalyst
}

Yi Wang, Hongming Li, Yong-Qiang Wang, Yan Liu, Bruce M. Foxman, Li Deng*

Department of Chemistry, Brandeis University

Waltham, Massachusetts, 02454-9110

\section{Supporting Information}

General Information. ${ }^{1} \mathrm{H}$ and ${ }^{13} \mathrm{C}$ NMR spectra were recorded on a Varian instrument (400 MHz and $100 \mathrm{MHz}$, respectively) and internally referenced to tetramethylsilane signal or residual protio solvent signals. Data for ${ }^{1} \mathrm{H}$ NMR are recorded as follows: chemical shift $(\delta, \mathrm{ppm})$, multiplicity ( $\mathrm{s}$, singlet; $\mathrm{d}$, doublet; t, triplet; q, quartet; $\mathrm{m}$, multiplet), intergration, coupling constant $(\mathrm{Hz})$. Data for ${ }^{13} \mathrm{C}$ NMR are reported in terms of chemical shift $(\delta, \mathrm{ppm})$. Infrared spectra were recorded on a Perkin Elmer FT-IR Spectrometer and are reported in frequency of absorption. High resolution mass spectra for all the new compounds were done by either a Micromass 70-VSEB instrument (CI) or a Micromass Q-Tof instrument (ESI). Specific rotations were measured on a Jasco Digital Polarimeter.

High performance liquid chromatography (HPLC) analysis was performed on a HewlettPackard 1100 Series instrument equipped with a quaternary pump, using a Daicel Chiralcel OJ, OD Column ( $250 \times 4.6 \mathrm{~mm})$ or Chiralpak AD, AS Column $(250 \times 4.6 \mathrm{~mm})$, and Regis $(R, R)$-Whelk-O1 $(250 \times 4.6 \mathrm{~mm})$ columns. UV absorption was monitored at 220 or $254 \mathrm{~nm}$. 
Materials: 2-pyrone $3 \mathbf{a}^{1}, \mathbf{3} \mathbf{d}^{2}, 3 \mathbf{e}^{2}$ and dienophile $\mathbf{4} \mathbf{B}^{3}, \mathbf{4 F ^ { 4 }}$ were prepared according to literature methods. Dienophile 4A, 4D were purchased from TCI, 4C, 4E, 4G, 4H were purchased from Aldrich and used without further purification.<smiles>O=c1occcc1O</smiles>

$3 a$<smiles></smiles>

$3 b$<smiles>Cc1ccoc(=O)c1O</smiles>

3c<smiles>O=c1occc(Cl)c1O</smiles>

$3 d$<smiles>O=c1occc(Br)c1O</smiles>

$3 e$<smiles>CCOC(=O)/C=C/C(=O)c1ccccc1</smiles>

$4 \mathrm{~A}$<smiles>N#CC=C[N+]=[N-]</smiles>

4E<smiles>CCOC(=O)/C=C/C(=O)c1ccc(Br)cc1</smiles>

4B<smiles>O=C(/C=C/C(=O)c1ccccc1)c1ccccc1</smiles>

4C<smiles>C=CC#N</smiles>

4G<smiles>C=C(C)C(C)=O</smiles>

4D

S-Figure 1. The Structures of 2-Pyrones 3 and Dienophiles 4.

(For structure of cinchona alkaloid catalysts, see S-Figure 2). DABCO, DHQD-PHN were purchased from Aldrich and used without further purification. 1a ${ }^{5}$, QD-1 $\mathbf{b}^{5}$, QD-1c ${ }^{6}$, QD-1d ${ }^{7}$ and $\mathbf{2}^{8}, \beta-\mathrm{ICD}^{9}$ were synthesized according to the reported methods.

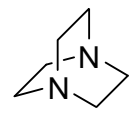

$\mathrm{DABCO}$

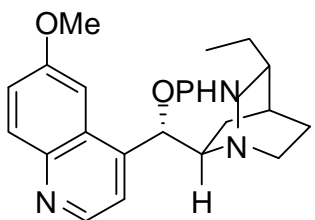

DHQD-PHN<smiles>CCC12CCC(CC1)N1CCC2CC1c1ccnc2ccc(O)cc12</smiles>

$\beta$-ICD

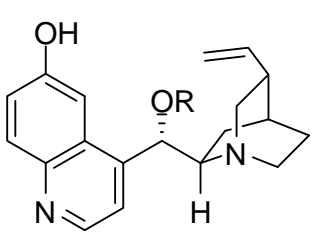

$\mathrm{R}=\mathrm{Bn}, \mathrm{QD}-\mathbf{1} \mathbf{b}$

$\mathrm{R}=\mathrm{Ac}, \mathrm{QD}-\mathbf{- 1 c}$

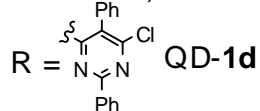<smiles>C=C(Cl)C#N</smiles>

4H 


\section{Preparation of 2-pyrone $3 b^{10}, 3 c^{11}$}

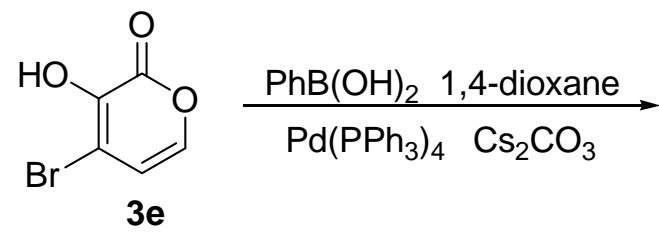<smiles>O=c1occc(-c2ccccc2)c1O</smiles>

Under Argon atmosphere, a solution of $3 \mathbf{e}(85.0 \mathrm{mg}, 0.50 \mathrm{mmol})$ and $\mathrm{Pd}\left(\mathrm{PPh}_{3}\right)_{4}(62.0 \mathrm{mg}$, $0.05 \mathrm{mmol})$ in anhydrous 1,6-dioxane $(2.0 \mathrm{~mL})$ was stirred at room temperature for $0.5 \mathrm{~h}$, then phenylboronic acid $(77.0 \mathrm{mg}, 0.66 \mathrm{mmol})$ and cesium carbonate $(211.0 \mathrm{mg}, 1.4$ mmol) were added. The resulting mixture was heated at $80^{\circ} \mathrm{C}$ for $24 \mathrm{~h}$, and then cooled to room temperature. The mixture was diluted with diethyl ether, filtered through a celite pad, and concentrated. The crude product was purified by column chromatography (hexanes/ethyl acetate $=4 / 1)$ to furnish $\mathbf{3 b}$ as a white solid $\left(28.0 \mathrm{mg}, 34 \%\right.$ yield). ${ }^{1} \mathrm{H}$ NMR (400 MHz, $\left.\mathrm{CDCl}_{3}\right) \delta 6.47(\mathrm{~d}, J=5.6 \mathrm{~Hz}, 1 \mathrm{H}), 6.53(\mathrm{~s}, 1 \mathrm{H}), 7.22(\mathrm{~d}, J=5.2 \mathrm{~Hz}$, 1H), 7.42-7.50 (m, 3H), $7.73(\mathrm{~d}, J=7.2 \mathrm{~Hz}, 2 \mathrm{H}) ;{ }^{13} \mathrm{C} \mathrm{NMR}\left(100 \mathrm{MHz}, \mathrm{CDCl}_{3}\right) \delta 109.5$, 126.7, 129.2, 129.3, 130.0, 133.7, 138.5, 141.6, 163.0; IR (neat) $\vee 3325,1672$; HRMS $\left(\mathrm{CI} /[\mathrm{M}+\mathrm{H}]^{+}\right)$Calcd. for: $\mathrm{C}_{11} \mathrm{H}_{9} \mathrm{O}_{3}: 189.0552$. Found: 189.0551 .
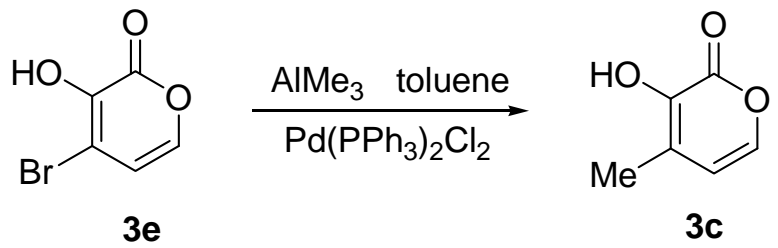

A solution of $3 \mathbf{e}(100.0 \mathrm{mg}, 0.625 \mathrm{mmol}), \mathrm{PdCl}_{2}\left(\mathrm{PPh}_{3}\right)_{2}(12.0 \mathrm{mg}, 0.017 \mathrm{mmol}), \mathrm{AlMe}_{3}$ $(0.35 \mathrm{~mL}, 2 \mathrm{M}$ solution in hexanes) and $5 \mathrm{~mL}$ of anhydrous toluene in a seal tube was stirred for $5 \mathrm{~h}$ at $100{ }^{\circ} \mathrm{C}$. The reaction mixture was then concentrated and directly purified by column chromatography (hexanes/ethyl acetate $=2 / 1$ ) to furnish $3 \mathbf{c}$ as a white solid (54.6 mg, $71 \%$ yield). ${ }^{1} \mathrm{H}$ NMR (400 MHz, $\left.\mathrm{CDCl}_{3}\right) \delta 2.13(\mathrm{~s}, 3 \mathrm{H}), 6.11(\mathrm{~d}, J=5.6 \mathrm{~Hz}$, 1H), $6.45(\mathrm{~s}, 1 \mathrm{H}), 7.10(\mathrm{~d}, J=5.2 \mathrm{~Hz}, 1 \mathrm{H}) ;{ }^{13} \mathrm{C} \mathrm{NMR}\left(100 \mathrm{MHz}, \mathrm{CDCl}_{3}\right) 14.56,111.13$, 126.75, 138.99, 140.67, 161.21; IR (neat) v 3335, 1651, 1668; HRMS (CI/[M+H] $]^{+}$Calcd. for: $\mathrm{C}_{6} \mathrm{H}_{7} \mathrm{O}_{3}: 127.0395$. Found: 127.0394 . 


\section{Preparation of racemic Diels-Alder reaction adducts}

At room temperature to a solution of $\mathbf{3}(0.05 \mathrm{mmol})$ in ethyl acetate $(0.25 \mathrm{~mL})$ was added 4 (2-4 equiv.), and $\mathrm{DABCO}$ or $\mathrm{Et}_{3} \mathrm{~N}$ (20 mol\%). After the reaction went to completion, the crude reaction mixture was passed through a short plug of silica gel, and the silica gel plug was washed with diethyl ether (3.0-4.0 mL). After removal of the solvent, the residue was purified by preparative TLC, and subject to HPLC analysis.

\section{General reaction procedure for asymmetric Diels-Alder reaction}

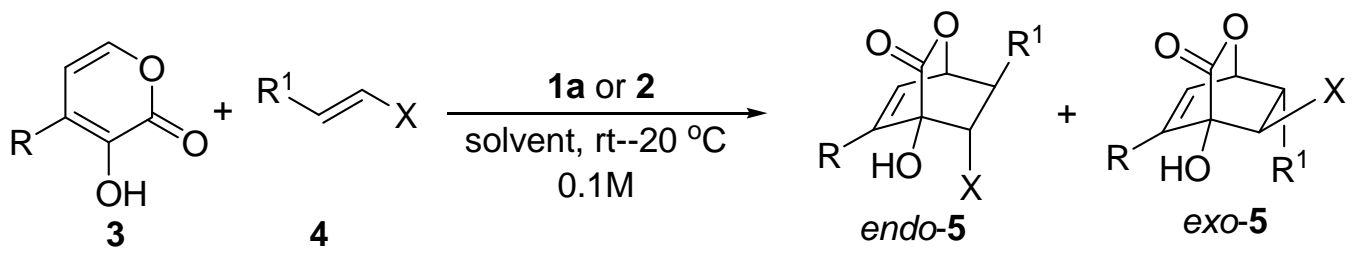

At room temperature, to a solution of 2-pyrones $3(0.3 \mathrm{mmol})$ and catalyst $1 \mathrm{a}$ or $2(0.015$ $0.030 \mathrm{mmol}, 5-10 \mathrm{~mol} \%$ ) in solvent ( $3.0 \mathrm{~mL}$, as specified below) was added dienophiles 4 (0.6-1.2 mmol, 2-4 equiv.). The reaction mixture was kept at certain reaction temperature for 11-72 $\mathrm{h}$ as specified in the following experimental section. The crude reaction mixture was passed through a short plug of silica gel for the removal of the catalyst, and the silica gel plug was washed with diethyl ether or ethyl acetate (3.0-4.0 $\mathrm{mL}$ ). The eluent was concentrated in vacuo, and the residue was subjected to silica gel flash chromatography.

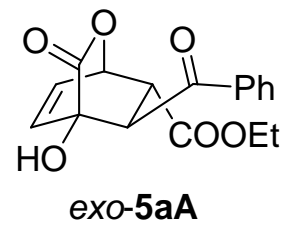

\section{Ethyl 8-benzoyl-4-hydroxy-3-oxo-2-oxabicyclo[2.2.2]oct-5-ene-7- carboxylate (5aA) \\ QD-1a-catalyzed (7.3 mg, $0.015 \mathrm{mmol}, 5 \mathrm{~mol} \%)$ reaction of $3 \mathbf{a}(33.6$ $\mathrm{mg}, 0.3 \mathrm{mmol})$ and $4 \mathrm{a}(112.4 \mathrm{mg}, 0.6 \mathrm{mmol}, 2.0$ equiv.) in diethyl}

ether $(3.0 \mathrm{~mL})$ was run at room temperature for $17 \mathrm{~h}$ to furnish the crude product \{exo:endo $=93: 7$, determined by integration of one set of ${ }^{1} \mathrm{H}$ NMR signals $\left[\delta_{\text {major }}=5.57\right.$ ppm $\left.\left.(\mathrm{m}), \delta_{\text {minor }}=5.70 \mathrm{ppm}(\mathrm{d})\right]\right\}$. The crude product was purified by flash chromatography (silica gel, hexanes/ethyl acetate $=3 / 1$ ) to give pure exo-5aA (major diastereomers) as a light brown oil $(82.0 \mathrm{mg}, 87 \%$ yield) in $94 \%$ ee [determined by HPLC, $(R, R)$-Whelk-O1, hexanes: isopropanol $=90: 10,1.0 \mathrm{~mL} / \mathrm{min}, \lambda=220 \mathrm{~nm}, \mathrm{t}$ 
$($ major $)=21.1 \mathrm{~min}, \mathrm{t}($ minor $)=19.7 \mathrm{~min}] .[\alpha]^{\mathrm{D}}{ }_{25}=+9.0\left(c 0.51, \mathrm{CHCl}_{3}\right) ;{ }^{1} \mathrm{H} \mathrm{NMR}(400$ $\left.\mathrm{MHz}, \mathrm{CDCl}_{3}\right) \delta 1.21(\mathrm{t}, J=6.8 \mathrm{~Hz}, 3 \mathrm{H}), 3.60(\mathrm{t}, J=4.8 \mathrm{~Hz}, 1 \mathrm{H}), 3.75(\mathrm{~s}, 1 \mathrm{H}), 4.16(\mathrm{q}, J$ $=7.2 \mathrm{~Hz}, 2 \mathrm{H}), 4.44(\mathrm{~d}, J=6.4 \mathrm{~Hz}, 1 \mathrm{H}), 5.57(\mathrm{~m}, 1 \mathrm{H}), 6.48(\mathrm{dd}, J=4.8,7.6 \mathrm{~Hz}, 1 \mathrm{H})$, $6.61(\mathrm{~d}, J=7.6 \mathrm{~Hz}, 1 \mathrm{H}), 7.48(\mathrm{t}, J=7.2 \mathrm{~Hz}, 2 \mathrm{H}), 7.61(\mathrm{t}, J=7.6 \mathrm{~Hz}, 1 \mathrm{H}), 8.07(\mathrm{~d}, J=$ $8.0 \mathrm{~Hz}, 2 \mathrm{H}) ;{ }^{13} \mathrm{C}$ NMR $\left(100 \mathrm{MHz}, \mathrm{CDCl}_{3}\right) \delta 13.93,48.20,49.30,62.00,72.72,76.39$, 128.61, 129.02, 129.14, 133.97, 136.91, 138.91, 169.28, 173.22, 199.07; IR (neat) v 3457, 1770, 1732, 1680; HRMS (ESI/[M+Na] $]^{+}$) Calcd. for: $\mathrm{C}_{17} \mathrm{H}_{16} \mathrm{O}_{6} \mathrm{Na}: 339.0845$. Found: 339.0841 .

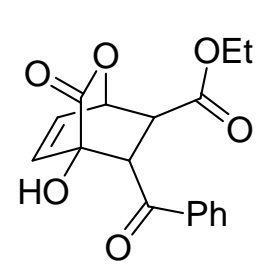

endo-5aA

endo-5aA (minor diastereomers) obtained from the same reaction was isolated as a colorless solid (7.0 mg, 7\% yield) in 54\% ee [determined by HPLC, $(R, R)$-Whelk-O1, hexanes: isopropanol $=90: 10,1.0 \mathrm{~mL} / \mathrm{min}, \lambda$ $=220 \mathrm{~nm}, \mathrm{t}($ major $)=13.6 \mathrm{~min}, \mathrm{t}($ minor $)=15.2 \mathrm{~min}] .[\alpha]_{25}^{\mathrm{D}}=+29.1(\mathrm{c}$ $\left.0.22, \mathrm{CHCl}_{3}\right) ;{ }^{1} \mathrm{H} \mathrm{NMR}\left(400 \mathrm{MHz}, \mathrm{CDCl}_{3}\right) \delta 1.28(\mathrm{t}, J=7.2 \mathrm{~Hz}, 3 \mathrm{H})$, $3.30(\mathrm{~d}, J=4.0 \mathrm{~Hz}, 1 \mathrm{H}), 3.73(\mathrm{~s}, 1 \mathrm{H}), 4.25(\mathrm{q}, J=6.4 \mathrm{~Hz}, 2 \mathrm{H}), 4.49(\mathrm{~d}, J=4.8 \mathrm{~Hz}, 1 \mathrm{H})$, $5.70(\mathrm{~d}, J=4.8 \mathrm{~Hz}, 1 \mathrm{H}), 6.44(\mathrm{~d}, J=8.0 \mathrm{~Hz}, 1 \mathrm{H}), 6.54(\mathrm{dd}, J=4.8,7.6 \mathrm{~Hz}, 1 \mathrm{H}), 7.49(\mathrm{t}$, $J=8.0 \mathrm{~Hz}, 2 \mathrm{H}), 7.61(\mathrm{t}, J=7.2 \mathrm{~Hz}, 1 \mathrm{H}), 8.03(\mathrm{~d}, J=8.0 \mathrm{~Hz}, 2 \mathrm{H}) ;{ }^{13} \mathrm{C} \mathrm{NMR}(100 \mathrm{MHz}$, $\left.\mathrm{CDCl}_{3}\right) \delta 14.07,44.95,50.16,62.31,75.88,76.19,128.44,128.65,129.17,133.87$, 136.23, 137.03, 169.38, 173.76, 196.88; IR (neat) $\vee$ 3460, 1767, 1733, 1682; HRMS $\left(\mathrm{ESI} /[\mathrm{M}+\mathrm{H}]^{+}\right)$Calcd. for: $\mathrm{C}_{17} \mathrm{H}_{17} \mathrm{O}_{6}: 317.1025$. Found: 317.1035 .

Q-1a-catalyzed reaction under the same conditions for $17 \mathrm{~h}$ furnished crude ent-5aA (exo:endo = 94:6). After chromatographic purification, ent-exo-5aA was obtained in 90\% yield ( $85.0 \mathrm{mg}, 87 \%$ ee), and ent-endo-5aA was obtained in 5\% yield (5.0 $\mathrm{mg}, 23 \%$ ee).

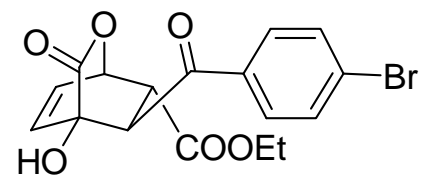

exo-5aB
Ethyl 8-(4-bromobenzoyl)-4-hydroxy-3-oxo-2-oxabicyclo -[2.2.2]oct-5-ene-7-carboxylate (5aB)

QD-1a-catalyzed (7.3 $\mathrm{mg}, 0.015 \mathrm{mmol}, 5 \mathrm{~mol} \%)$ reaction of 3a $(33.6 \mathrm{mg}, 0.3 \mathrm{mmol})$ and 4B $(170.0 \mathrm{mg}, 0.6 \mathrm{mmol}, 2.0$ equiv.) in diethyl ether $(3.0 \mathrm{~mL})$ was run at room temperature for $17 \mathrm{~h}$ to furnish the crude product \{exo: endo $=91: 9$, determined by integration of one set of ${ }^{1} \mathrm{H}$ NMR signals 
$\left[\left(\delta_{\text {major }}=5.55-5.67 \mathrm{ppm}(\mathrm{m}), \delta_{\text {minor }}=5.70 \mathrm{ppm}(\mathrm{d})\right]\right\}$. The crude product was purified by flash chromatography (silica gel, hexanes/ethyl acetate $=5 / 1$ ) to give pure exo-5aB (major diastereomers) as a white solid (107.3 $\mathrm{mg}, 91 \%$ yield) in 91\% ee [determined by HPLC, Chiralpak AD, hexanes: isopropanol $=80: 20,1.0 \mathrm{~mL} / \mathrm{min}, \lambda=220 \mathrm{~nm}, \mathrm{t}$ (major) $=19.4$ min, $\mathrm{t}($ minor $)=15.9 \mathrm{~min}] .[\alpha]_{25}^{\mathrm{D}}=-2.0\left(\right.$ c $\left.0.50, \mathrm{CHCl}_{3}\right) ;{ }^{1} \mathrm{H} \mathrm{NMR}(400 \mathrm{MHz}$, $\left.\mathrm{CDCl}_{3}\right) \delta 1.21(\mathrm{t}, J=6.8 \mathrm{~Hz}, 3 \mathrm{H}), 3.58(\mathrm{dd}, J=3.6,5.2 \mathrm{~Hz}, 1 \mathrm{H}), 3.78(\mathrm{~s}, 1 \mathrm{H}), 4.15(\mathrm{q}, J$ $=7.6 \mathrm{~Hz}, 2 \mathrm{H}), 4.35(\mathrm{~d}, J=6.0 \mathrm{~Hz}, 1 \mathrm{H}), 5.55-5.57(\mathrm{~m}, 1 \mathrm{H}), 6.47(\mathrm{dd}, J=4.8,8.0 \mathrm{~Hz}, 1 \mathrm{H})$, $6.58(\mathrm{dd}, J=1.2,8.0 \mathrm{~Hz}, 1 \mathrm{H}), 7.61(\mathrm{~d}, J=8.0 \mathrm{~Hz}, 2 \mathrm{H}), 7.91(\mathrm{~d}, J=8.4 \mathrm{~Hz}, 2 \mathrm{H}) ;{ }^{13} \mathrm{C}$ NMR $\left(100 \mathrm{MHz}, \mathrm{CDCl}_{3}\right) \delta 14.66,48.91,49.96,62.81,73.39,77.07,129.91,130.15$, 131.22, 132.65, 136.39, 139.64, 169.89, 137.75, 198.89; IR (neat) v 3446, 1770, 1734, 1697; HRMS (ESI/[M+H] ${ }^{+}$) Calcd. for: $\mathrm{C}_{17} \mathrm{H}_{16} \mathrm{O}_{6} \mathrm{Br}: 395.0130$. Found: 395.0129 .

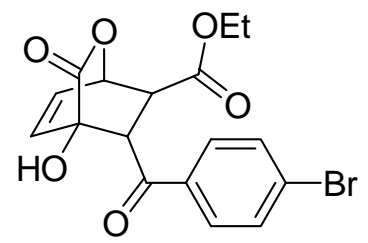

endo-5aB

endo-5aB (minor diastereomers) obtained from the same reaction was isolated as a colorless oil $(9.0 \mathrm{mg}, 8 \%$ yield $)$ in $53 \%$ ee [determined by HPLC, Chiralpak AD, hexanes: isopropanol $=$ $60: 40,1.0 \mathrm{~mL} / \mathrm{min}, \lambda=220 \mathrm{~nm}, \mathrm{t}$ (major) $=9.1 \mathrm{~min}, \mathrm{t}$ (minor) $=$ $14.7 \mathrm{~min}] .[\alpha]_{25}^{\mathrm{D}}=+26.0\left(\right.$ c $\left.0.50, \mathrm{CHCl}_{3}\right) ;{ }^{1} \mathrm{H}$ NMR $(400 \mathrm{MHz}$, $\left.\mathrm{CDCl}_{3}\right) \delta 1.28(\mathrm{t}, J=7.2 \mathrm{~Hz}, 3 \mathrm{H}), 3.31(\mathrm{~d}, J=4.4 \mathrm{~Hz}, 1 \mathrm{H}), 3.78(\mathrm{~s}, 1 \mathrm{H}), 4.24(\mathrm{q}, J=5.6$ $\mathrm{Hz}, 2 \mathrm{H}), 4.42(\mathrm{~d}, J=4.4 \mathrm{~Hz}, 1 \mathrm{H}), 5.70(\mathrm{~d}, J=4.8 \mathrm{~Hz}, 1 \mathrm{H}), 6.40(\mathrm{~d}, J=7.2 \mathrm{~Hz}, 1 \mathrm{H}), 6.55$ $(\mathrm{dd}, J=5.2,8.0 \mathrm{~Hz}, 1 \mathrm{H}), 7.63(\mathrm{~d}, J=8.4 \mathrm{~Hz}, 2 \mathrm{H}), 7.90(\mathrm{~d}, J=8.4 \mathrm{~Hz}, 2 \mathrm{H}) ;{ }^{13} \mathrm{C} \mathrm{NMR}$ $\left(100 \mathrm{MHz}, \mathrm{CDCl}_{3}\right) \delta 14.77,45.60,50.76,63.10,76.53,76.85,129.43,130.07,131.42$, 132.66, 136.47, 136.65, 170.00, 174.34, 196.64; IR (neat) v 3447, 1770, 1773, 1697; HRMS (ESI/[M+H] $]^{\dagger}$ ) Calcd. for: $\mathrm{C}_{17} \mathrm{H}_{16} \mathrm{O}_{6} \mathrm{Br}$ : 395.0130. Found: 395.0133 .

Q-1a-catalyzed under the same conditions for $17 \mathrm{~h}$ furnished crude ent-5aB (exo: endo $=$ 94:6). After chromatographic purification, ent-exo-5aB was obtained in 93\% yield (110.2 $\mathrm{mg}, 83 \%$ ee), and ent-endo-5aB was obtained in $6 \%$ yield $(7.1 \mathrm{mg}, 26 \%$ ee). 


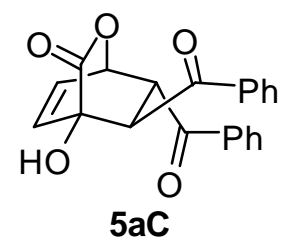

(4-hydroxy-3-oxo-2-oxabicyclo[2.2.2]oct-5-ene-7,8-diyl)bis(phenylmethanone) (5aC)

QD-1a-catalyzed (7.3 mg, $0.015 \mathrm{mmol}, 5 \mathrm{~mol} \%)$ reaction of 3a (33.6 $\mathrm{mg}, 0.3 \mathrm{mmol})$ and $4 \mathrm{C}(141.6 \mathrm{mg}, 0.6 \mathrm{mmol}, 2.0$ equiv.) in diethyl ether $(3.0 \mathrm{~mL})$ was run at room temperature for $12 \mathrm{~h}$ to furnish the crude product \{exo: endo $=93: 7$, determined by integration of one set of ${ }^{1} \mathrm{H}$ NMR signals $\left[\delta_{\text {major }}=4.54-4.57\right.$ ppm $\left.\left.(\mathrm{m}), \delta_{\text {minor }}=4.77 \mathrm{ppm}(\mathrm{d})\right]\right\}$. The crude product was purified by flash chromatography (silica gel, hexanes/ethyl acetate $=3 / 1$ ) to give adduct $\mathbf{5 a C}$ (mixture of endo and exo products) as a colorless oil $(104.0 \mathrm{mg}, 100 \%$ yield, $\mathrm{dr}=93: 7)$ in $90 \%$ ee (exo-5aC, major diastereomer) [determined by HPLC, Chiralpak AD, hexanes: isopropanol $=80: 20,1.0 \mathrm{~mL} / \mathrm{min}, \lambda=220 \mathrm{~nm}, \mathrm{t}$ (major) $=39.6 \mathrm{~min}, \mathrm{t}$ (minor) $=30.1$ min]. ${ }^{1} \mathrm{H}$ NMR (400 MHz, $\mathrm{CDCl}_{3}$ ) of the major diastereomer, $\delta 3.64(\mathrm{~s}, 1 \mathrm{H}), 4.54-4.57$ (m, 2H), 5.54-5.55 (m, 1H), $6.41(\mathrm{dd}, J=6.4,8.0 \mathrm{~Hz}, 1 \mathrm{H}), 6.59$ (dd, $J=2.0,8.0 \mathrm{~Hz}, 1 \mathrm{H})$, $7.40(\mathrm{dd}, J=7.2,15.2 \mathrm{~Hz}, 4 \mathrm{H}), 7.53-7.57(\mathrm{~m}, 2 \mathrm{H}), 7.81(\mathrm{~d}, J=8.0 \mathrm{~Hz}, 2 \mathrm{H}), 7.92(\mathrm{~d}, J=$ $7.2 \mathrm{~Hz}, 2 \mathrm{H}) ;{ }^{13} \mathrm{C}$ NMR $\left(100 \mathrm{MHz}, \mathrm{CDCl}_{3}\right)$ of the major diastereomer, $\delta 47.70,52.88$, $73.55,76.68,128.42,128.68,128.95,129.10,134.05,134.25,134.76,137.00,137.59$, 137.45, 195.43, 199.57; IR (neat) v 3463, 1767, 1681; HRMS (ESI/[M+H] ${ }^{+}$) Calcd. for: $\mathrm{C}_{21} \mathrm{H}_{17} \mathrm{O}_{5}$ : 349.1076. Found: 349.1078 .

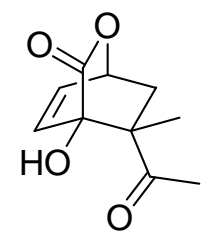

8-acetyl-4-hydroxy-8-methyl-2-oxabicyclo[2.2.2]oct-5-en-3-one (5aD)

endo-5aD

QD-1a-catalyzed (14.6 mg, $0.03 \mathrm{mmol}, 10 \mathrm{~mol} \%)$ reaction of $3(33.6 \mathrm{mg}$, $0.3 \mathrm{mmol})$ and $4 \mathrm{D}(118 \mu \mathrm{L}, 1.2 \mathrm{mmol}, 4.0$ equiv. $)$ in diethyl ether $(3.0 \mathrm{~mL})$ was run at room temperature for $36 \mathrm{~h}$ to furnish the crude product \{exo:endo $=24: 76$, determined by integration of one set of ${ }^{1} \mathrm{H}$ NMR signals $\left[\delta_{\text {major }}=2.75\right.$ $\left.\left.(\mathrm{dd}), \delta_{\text {major }}=2.69(\mathrm{dd})\right]\right\}$. The crude product was purified by flash chromatography (silica gel, hexanes/ethyl acetate $=2 / 1-1 / 1$ ) to give endo-5aD (major diastereomers) as a white solid (38.0 mg, 65\% yield) in 91\% ee [determined by HPLC, Chiralcel OD, hexanes: isopropanol $=95: 5,1.0 \mathrm{~mL} / \mathrm{min}, \lambda=220 \mathrm{~nm}, \mathrm{t}$ (major) $=18.4 \mathrm{~min}, \mathrm{t}$ (minor) $=20.3 \mathrm{~min}]$. $[\alpha]_{25}^{\mathrm{D}}=-20.0\left(c \quad 0.50, \mathrm{CHCl}_{3}\right) ;{ }^{1} \mathrm{H}$ NMR $\left(400 \mathrm{MHz}, \mathrm{CDCl}_{3}\right) \delta 1.35(\mathrm{~s}, 3 \mathrm{H}), 1.93(\mathrm{dd}, J=$ 3.6, $13.6 \mathrm{~Hz}, 1 \mathrm{H}), 2.31$ (s, 3H), $2.76(\mathrm{~d}, J=14.0 \mathrm{~Hz}, 1 \mathrm{H}), 4.02(\mathrm{~s}, 1 \mathrm{H}), 5.24(\mathrm{~m}, 1 \mathrm{H})$, $6.32(\mathrm{~d}, J=8.0 \mathrm{~Hz}, 1 \mathrm{H}), 6.39(\mathrm{dd}, J=5.6,8.0 \mathrm{~Hz}, 1 \mathrm{H}) ;{ }^{13} \mathrm{C} \mathrm{NMR}\left(100 \mathrm{MHz}, \mathrm{CDCl}_{3}\right) \delta$ 
$21.00,27.57,37.80,51.27,74.15,78.34,130.12,135.50,174.39,207.66$; IR (neat) $v$ 3460, 3420, 1743, 1692; HRMS (CI/[M+H] $]^{+}$Calcd. for: $\mathrm{C}_{10} \mathrm{H}_{13} \mathrm{O}_{4}$ : 197.0814. Found: 197.0818 .

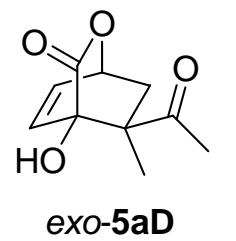

exo-5aD (minor diastereomers) obtained from the same reaction was isolated as a white solid (9.4 $\mathrm{mg}, 16 \%$ yield) in $95 \%$ ee [determined by HPLC, Chiralpak AS hexane:isopropanol $=70: 30,1.0 \mathrm{~mL} / \mathrm{min}, \lambda=220 \mathrm{~nm}$, $\mathrm{t}($ major $)=14.8 \mathrm{~min}, \mathrm{t}($ minor $)=18.8 \mathrm{~min}] .[\alpha]_{25}^{\mathrm{D}}=+16.6\left(\right.$ c $\left.0.47, \mathrm{CHCl}_{3}\right)$;

${ }^{1} \mathrm{H}$ NMR (400 MHz, $\left.\mathrm{CDCl}_{3}\right) \delta 1.44(\mathrm{~s}, 3 \mathrm{H}), 1.56(\mathrm{dd}, J=1.2,14.4 \mathrm{~Hz}, 1 \mathrm{H}), 2.28(\mathrm{~s}, 3 \mathrm{H})$, $2.69(\mathrm{dd}, J=4.4,14.0 \mathrm{~Hz}, 1 \mathrm{H}), 3.81(\mathrm{~s}, 1 \mathrm{H}), 5.20(\mathrm{~m}, 1 \mathrm{H}), 6.38(\mathrm{~d}, J=6.8 \mathrm{~Hz}, 1 \mathrm{H}), 6.46$ $(\mathrm{dd}, J=4.8,8.0 \mathrm{~Hz}, 1 \mathrm{H}) ;{ }^{13} \mathrm{C}$ NMR $\left(100 \mathrm{MHz}, \mathrm{CDCl}_{3}\right) \delta 20.52,28.45,38.92,52.19$, 72.48, 79.13, 129.86, 137.00, 174.38, 211.33; IR (neat) $v$ 3456, 1756, 1701; HRMS $\left(\mathrm{CI} /[\mathrm{M}+\mathrm{H}]^{+}\right)$Calcd. for: $\mathrm{C}_{10} \mathrm{H}_{13} \mathrm{O}_{4}:$ 197.0814. Found: 197.0813 .

Q-1a-catalyzed reaction under the same conditions for $96 \mathrm{~h}$ furnished crude ent-5aD (exo:endo $=26: 74)$. After chromatographic purification, ent-endo-5aD was obtained in $63 \%$ yield (37.4 $\mathrm{mg}, 90 \%$ ee), and ent-endo-5aD was obtained in $17 \%$ yield $(10.0 \mathrm{mg}$, $93 \%$ ee).

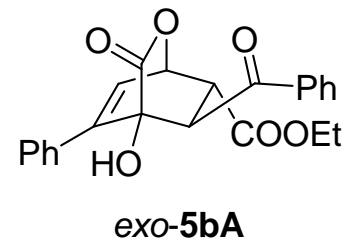

ethyl 8-benzoyl-4-hydroxy-3-oxo-5-phenyl-2-oxabicyclo[2.2.2]oct-5-ene-7-carboxylate (5bA)

QD-1a-catalyzed (7.3 $\mathrm{mg}, 0.015 \mathrm{mmol}, 5 \mathrm{~mol} \%$ ) reaction of $\mathbf{3 b}$ (56.4 mg, $0.3 \mathrm{mmol})$ and $\mathbf{4 A}(112.4 \mathrm{mg}, 0.6 \mathrm{mmol}, 2.0$ equiv.) in diethyl ether $(3.0 \mathrm{~mL})$ was run at $0{ }^{\circ} \mathrm{C}$ for $17 \mathrm{~h}$ to furnish the crude product \{exo:endo= 95:5, determined by integration of one set of ${ }^{1} \mathrm{H}$ NMR signals $\left[\delta_{\text {major }}=6.48(\mathrm{~d}), \delta_{\text {minor }}=\right.$ $6.59 \mathrm{ppm}(\mathrm{d})]\}$. The crude product was purified by flash chromatography (silica gel, hexanes/ethyl acetate $=5 / 1$ ) to give pure exo-5bA (major diastereomers) as colorless oil ( $99.3 \mathrm{mg}, 84 \%$ yield) in $85 \%$ ee [determined by HPLC, $(R, R)$-Whelk-O1, hexanes: isopropanol $=90: 10,0.8 \mathrm{~mL} / \mathrm{min}, \lambda=220 \mathrm{~nm}, \mathrm{t}$ (major) $=34.8 \mathrm{~min}, \mathrm{t}$ (minor) $=28.8$ $\min ] .[\alpha]_{25}^{\mathrm{D}}=+23.0\left(\right.$ c $\left.1.70, \mathrm{CHCl}_{3}\right) ;{ }^{1} \mathrm{H} \mathrm{NMR}\left(400 \mathrm{MHz}, \mathrm{CDCl}_{3}\right) \delta 1.23(\mathrm{t}, J=7.2 \mathrm{~Hz}$, $3 \mathrm{H}), 3.70(\mathrm{t}, J=4.8 \mathrm{~Hz}, 1 \mathrm{H}), 3.70(\mathrm{~s}, 1 \mathrm{H}), 4.18(\mathrm{q}, J=7.2 \mathrm{~Hz}, 2 \mathrm{H}), 4.65(\mathrm{~d}, J=6.0 \mathrm{~Hz}$, 
$1 \mathrm{H}), 5.66(\mathrm{t}, J=4.8 \mathrm{~Hz}, 1 \mathrm{H}), 6.48(\mathrm{~d}, J=5.6 \mathrm{~Hz}, 1 \mathrm{H}), 7.37-7.39(\mathrm{~m}, 3 \mathrm{H}), 7.45-7.48(\mathrm{~m}$, $2 \mathrm{H}), 7.50(\mathrm{t}, J=7.2 \mathrm{~Hz}, 2 \mathrm{H}), 7.62(\mathrm{t}, J=7.2 \mathrm{~Hz}, 1 \mathrm{H}), 8.10(\mathrm{~d}, J=7.2 \mathrm{~Hz}, 2 \mathrm{H}) ;{ }^{13} \mathrm{C}$ NMR $\left(100 \mathrm{MHz}, \mathrm{CDCl}_{3}\right) \delta 14.00,48.68,49.91,62.09,72.45,77.52,124.35,128.24$, $128.41,128.73,129.02,132.67,134.02,137.07,149.27,169.30,173.47,199.32$; IR (neat) $v$ 3465, 1767, 1732, 1682; HRMS (CI/[M+1] $)$ Calcd. for: $\mathrm{C}_{23} \mathrm{H}_{21} \mathrm{O}_{6}: 393.1338$. Found: 393.1333.

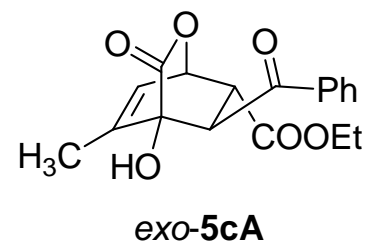

Ethyl 8-benzoyl-4-hydroxy-5-methyl-3-oxo-2-oxabicyclo[2.2.2]oct-5-ene-7-carboxylate (5cA)

QD-1a-catalyzed (7.3 $\mathrm{mg}, 0.015 \mathrm{mmol}, 5 \mathrm{~mol} \%$ ) reaction of 3c (37.8 $\mathrm{mg}, 0.3 \mathrm{mmol})$ and $\mathbf{4 A}(112.4 \mathrm{mg}, 0.6 \mathrm{mmol}, 2.0$ equiv.) in diethyl ether $(3.0 \mathrm{~mL})$ was run at $0{ }^{\circ} \mathrm{C}$ for $17 \mathrm{~h}$ to furnish the crude product \{exo:endo $=$ $88: 12$, determined by integration of one set of ${ }^{1} \mathrm{H}$ NMR signals $\left[\delta_{\text {major }}=6.15(\mathrm{~d}), \delta_{\text {minor }}=\right.$ 6.23-6.25 ppm (m)]\}. The crude product was purified by flash chromatography (silica gel, hexanes/ethyl acetate $=5: 1$ ) to give pure exo-5cA (major diastereomers) as white solid ( $86.0 \mathrm{mg}, 87 \%$ yield) in $82 \%$ ee [determined by HPLC, Chiralcel OD, hexanes: isopropanol $=85: 15,1.0 \mathrm{~mL} / \mathrm{min}, \lambda=220 \mathrm{~nm}, \mathrm{t}$ (major) $=13.0 \mathrm{~min}, \mathrm{t}$ (minor) $=16.5$ $\min ] .[\alpha]_{25}^{\mathrm{D}}=-2.3\left(\right.$ c $\left.1.00, \mathrm{CHCl}_{3}\right) ;{ }^{1} \mathrm{H}$ NMR $\left(400 \mathrm{MHz}, \mathrm{CDCl}_{3}\right) \delta 1.23(\mathrm{t}, J=7.6 \mathrm{~Hz}$, $3 \mathrm{H}), 1.97(\mathrm{~s}, 3 \mathrm{H}), 3.56-3.58(\mathrm{~m}, 2 \mathrm{H}), 4.17(\mathrm{q}, J=7.2 \mathrm{~Hz}, 2 \mathrm{H}), 4.36(\mathrm{~d}, J=5.2 \mathrm{~Hz}, 1 \mathrm{H})$, $5.48(\mathrm{t}, J=4.0 \mathrm{~Hz}, 1 \mathrm{H}), 6.15(\mathrm{~d}, J=3.6 \mathrm{~Hz}, 1 \mathrm{H}), 7.49(\mathrm{t}, J=7.6 \mathrm{~Hz}, 2 \mathrm{H}), 7.61(\mathrm{t}, J=$

$7.6 \mathrm{~Hz}, 1 \mathrm{H}), 8.07(\mathrm{~d}, J=7.6 \mathrm{~Hz}, 2 \mathrm{H}) ;{ }^{13} \mathrm{C} \mathrm{NMR}\left(100 \mathrm{MHz}, \mathrm{CDCl}_{3}\right) \delta 13.98,14.44,47.66$, $50.11,61.96,72.48,77.64,122.33,128.66,129.00,133.93,137.05,147.69,169.49$, 173.76, 199.47; IR (neat) $\vee$ 3460, 1766, 1732, 1681; HRMS (CI/[M+1] $\left.]^{+}\right)$Calcd. for: $\mathrm{C}_{18} \mathrm{H}_{19} \mathrm{O}_{6}:$ 331.1182. Found: 331.1181 .

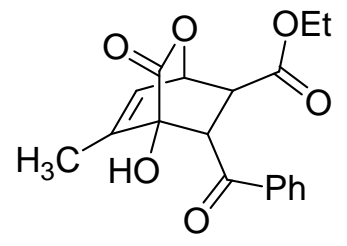

endo-5cA

endo-5cA (minor diastereomers) obtained from the same reaction was isolated as a light oil $(9.0 \mathrm{mg}, 9 \%$ yield) in $36 \%$ ee [determined by HPLC, Chiralcel OD, hexanes: isopropanol $=85: 15,0.8 \mathrm{~mL} / \mathrm{min}$, $\lambda=220 \mathrm{~nm}, \mathrm{t}$ (major) $=11.3 \mathrm{~min}, \mathrm{t}($ minor $)=21.0 \mathrm{~min}] .[\alpha]_{25}^{\mathrm{D}}=$ $+15.0\left(\right.$ c $\left.0.28, \mathrm{CHCl}_{3}\right) ;{ }^{1} \mathrm{H} \mathrm{NMR}\left(400 \mathrm{MHz}, \mathrm{CDCl}_{3}\right) \delta 1.27$ (t, $J=$

$7.6 \mathrm{~Hz}, 3 \mathrm{H}), 1.84(\mathrm{~d}, J=1.2 \mathrm{~Hz}, 3 \mathrm{H}), 3.32$ (d, $J=4.0 \mathrm{~Hz}, 1 \mathrm{H}), 3.71$ (s, $1 \mathrm{H}), 4.23$ (q, $J=$ 
$7.2 \mathrm{~Hz}, 2 \mathrm{H}), 4.47$ (d, $J=4.4 \mathrm{~Hz}, 1 \mathrm{H}), 5.61$ (d, $J=5.6 \mathrm{~Hz}, 1 \mathrm{H}), 6.23-6.25(\mathrm{~m}, 1 \mathrm{H}), 7.49$ (t, $J=7.6 \mathrm{~Hz}, 2 \mathrm{H}), 7.61(\mathrm{t}, J=7.6 \mathrm{~Hz}, 1 \mathrm{H}), 8.04(\mathrm{~d}, J=8.0 \mathrm{~Hz}, 2 \mathrm{H}) ;{ }^{13} \mathrm{C} \mathrm{NMR}(100 \mathrm{MHz}$, $\left.\mathrm{CDCl}_{3}\right) \delta 14.06,15.70,44.57,50.03,62.14,75.25,77.60,122.24,128.60,129.14,133.79$, 137.24, 144.42, 169.67, 174.24, 197.31; IR (neat) v 3461, 2982, 1760, 1740, 1732, 1682; HRMS (CI/[M+1] $]^{+}$Calcd. for: $\mathrm{C}_{18} \mathrm{H}_{19} \mathrm{O}_{6}: 331.1182$. Found: 331.1185 .

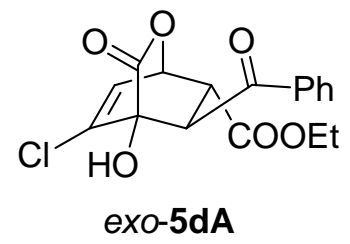

ethyl 8-benzoyl-5-chloro-4-hydroxy-3-oxo-2-xabicyclo[2.2.2]oct -5-ene-7-carboxylate (5dA)

QD-1a-catalyzed $(7.3 \mathrm{mg}, 0.015 \mathrm{mmol}, 5 \mathrm{~mol} \%)$ reaction of $3 \mathbf{d}$ (44.0 $\mathrm{mg}, 0.3 \mathrm{mmol}$ ) and 4A (112.4 $\mathrm{mg}, 0.6 \mathrm{mmol}, 2.0$ equiv.) in ethyl acetate $(3.0 \mathrm{~mL})$ was run at room temperature for $17 \mathrm{~h}$ to furnish the crude product $\left\{\right.$ exo:endo $=86: 14$, determined by integration of one set of ${ }^{1} \mathrm{H}$ NMR signals $\left[\delta_{\text {major }}=5.60\right.$ ppm $\left.\left.(\mathrm{dd}), \delta_{\text {minor }}=5.71 \mathrm{ppm}(\mathrm{d})\right]\right\}$. The crude product was purified by flash chromatography (silica gel, hexanes/ethyl acetate $=6: 1-4: 1$ ) to give pure exo-5dA (major diastereomers) as a colorless solid (81.0 mg, 77\% yield, $\mathrm{dr}>30: 1)$ in $84 \%$ ee [determined by HPLC, Chiralcel OJ, hexanes: isopropanol $=70: 30,0.8 \mathrm{~mL} / \mathrm{min}, \lambda=254 \mathrm{~nm}, \mathrm{t}$ (major) $=44.6 \mathrm{~min}, \mathrm{t}($ minor $)=28.9 \mathrm{~min}] .[\alpha]^{\mathrm{D}}{ }_{25}=+2.8\left(\right.$ c $\left.0.50, \mathrm{CHCl}_{3}\right) ;{ }^{1} \mathrm{H}$ NMR $(400 \mathrm{MHz}$, $\left.\mathrm{CDCl}_{3}\right) \delta 1.23(\mathrm{t}, J=7.2 \mathrm{~Hz}, 3 \mathrm{H}), 3.66(\mathrm{dd}, J=4.0,6.0 \mathrm{~Hz}, 1 \mathrm{H}), 3.75(\mathrm{~s}, 1 \mathrm{H}), 4.19(\mathrm{q}, J$ $=7.2 \mathrm{~Hz}, 2 \mathrm{H}), 4.49(\mathrm{~d}, J=5.6 \mathrm{~Hz}, 1 \mathrm{H}), 5.60(\mathrm{dd}, J=3.6,5.6 \mathrm{~Hz}, 1 \mathrm{H}), 6.52(\mathrm{~d}, J=5.6$ $\mathrm{Hz}, 1 \mathrm{H}), 7.50(\mathrm{t}, J=7.2 \mathrm{~Hz}, 2 \mathrm{H}), 7.63(\mathrm{t}, J=7.2 \mathrm{~Hz}, 1 \mathrm{H}), 8.07(\mathrm{~d}, J=7.6 \mathrm{~Hz}, 2 \mathrm{H}) ;{ }^{13} \mathrm{C}$ NMR $\left(100 \mathrm{MHz}, \mathrm{CDCl}_{3}\right) \delta 13.98,47.76,50.06,62.29,72.36,76.91,124.67,128.74$, 129.14, 134.22, 136.76, 140.57, 168.89, 171.50, 198.26; IR (neat) v 3442, 1773, 1771, 1731; HRMS (ESI/[M+Na] $]^{+}$) Calcd. for: $\mathrm{C}_{17} \mathrm{H}_{15} \mathrm{ClO}_{6} \mathrm{Na}: 373.0455$. Found: 373.0466 .

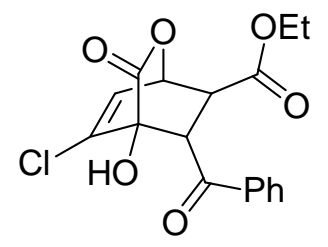

endo-5dA (minor diastereomers) obtained from the same reaction was isolated as a colorless solid $(8.1 \mathrm{mg}, 8 \%$ yield) and $1 \%$ ee [determined by HPLC, Chiralcel OJ, hexanes: isopropanol = 70:30, $0.8 \mathrm{~mL} / \mathrm{min}, \lambda=254 \mathrm{~nm}, \mathrm{t}$ (major) $=36.6 \mathrm{~min}, \mathrm{t}($ minor $)=19.5 \mathrm{~min}]$. endo-5dA $[\alpha]^{\mathrm{D}}{ }_{25}=-0.26\left(c 0.38, \mathrm{CHCl}_{3}\right) ;{ }^{1} \mathrm{H}$ NMR $\left(400 \mathrm{MHz}, \mathrm{CDCl}_{3}\right) \delta 1.28(\mathrm{t}$, $J=6.4 \mathrm{~Hz}, 3 \mathrm{H}), 3.39$ (d, $J=3.6 \mathrm{~Hz}, 1 \mathrm{H}), 3.84$ (brs, 1H), 4.25 (q, $J=7.2 \mathrm{~Hz}, 2 \mathrm{H}), 4.60$ $(\mathrm{d}, J=4.8 \mathrm{~Hz}, 1 \mathrm{H}), 5.71(\mathrm{~d}, J=4.8 \mathrm{~Hz}, 1 \mathrm{H}), 6.59(\mathrm{~d}, J=5.2 \mathrm{~Hz}, 1 \mathrm{H}), 7.50(\mathrm{t}, J=7.2 \mathrm{~Hz}$, 
2H), $7.62(\mathrm{t}, J=7.2 \mathrm{~Hz}, 1 \mathrm{H}), 8.05(\mathrm{~d}, J=7.2 \mathrm{~Hz}, 2 \mathrm{H}) ;{ }^{13} \mathrm{C} \mathrm{NMR}\left(100 \mathrm{MHz}, \mathrm{CDCl}_{3}\right) \delta$ $14.05,44.39,49.93,62.50,74.97,76.87,124.05,128.70,129.27,134.09,136.77,138.40$, 168.88, 171.81, 196.33; IR (neat) v 3446, 1772, 1734; HRMS (ESI/[M+Na] ${ }^{\dagger}$ ) Calcd. for: $\mathrm{C}_{17} \mathrm{H}_{15} \mathrm{ClO}_{6} \mathrm{Na}: 373.0455$. Found: 373.0474 .

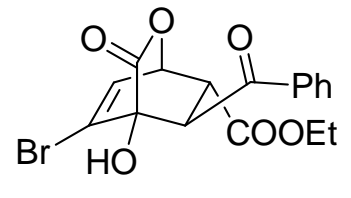

exo-5eA ethyl 8-benzoyl-5-bromo-4-hydroxy-3-oxo-2-oxabicyclo[2.2.2]oct-5-ene-7-carboxylate (5eA)

QD-1a-catalyzed (7.3 $\mathrm{mg}, 0.015 \mathrm{mmol}, 5 \mathrm{~mol} \%)$ reaction of $3 \mathbf{e}$ (57.3 $\mathrm{mg}, 0.3 \mathrm{mmol})$ and $\mathbf{4 A}(112.4 \mathrm{mg}, 0.6 \mathrm{mmol}, 2.0$ equiv.) in ethyl acetate $(3.0 \mathrm{~mL})$ was run at room temperature for $17 \mathrm{~h}$ to furnish the crude product $\left\{\right.$ exo:endo $=85: 15$, determined by integration of one set of ${ }^{1} \mathrm{H}$ NMR signals $\left[\delta_{\text {major }}=6.76\right.$ ppm (d), $\left.\left.\delta_{\text {minor }}=6.82 \mathrm{ppm}(\mathrm{d})\right]\right\}$. The crude product was purified by flash chromatography (silica gel, hexanes/ethyl acetate $=10 / 1-4 / 1$ ) to give pure exo-5eA (major diastereomers) as white solid $(89.1 \mathrm{mg}, 75 \%$ yield) in $83 \%$ ee [determined by HPLC, Chiralcel OJ, hexanes: isopropanol $=70: 30,0.8 \mathrm{~mL} / \mathrm{min}, \lambda=220 \mathrm{~nm}, \mathrm{t}$ (major) $=$ 53.0 min, $\mathrm{t}$ (minor) $=32.4 \mathrm{~min}] .[\alpha]_{25}^{\mathrm{D}}=+3.5\left(\right.$ c $\left.1.56, \mathrm{CHCl}_{3}\right) ;{ }^{1} \mathrm{H} \mathrm{NMR}(400 \mathrm{MHz}$, $\left.\mathrm{CDCl}_{3}\right) \delta 1.23(\mathrm{t}, J=7.2 \mathrm{~Hz}, 3 \mathrm{H}), 3.62-3.64(\mathrm{~m}, 1 \mathrm{H}), 3.84(\mathrm{~s}, 1 \mathrm{H}), 4.19$ (q, $J=7.2 \mathrm{~Hz}$, $2 \mathrm{H}), 4.44(\mathrm{~d}, J=5.6 \mathrm{~Hz}, 1 \mathrm{H}), 5.56-5.58(\mathrm{~m}, 1 \mathrm{H}), 6.76(\mathrm{~d}, J=5.6 \mathrm{~Hz}, 1 \mathrm{H}), 7.50(\mathrm{t}, J=$ $7.2 \mathrm{~Hz}, 2 \mathrm{H}), 7.63(\mathrm{t}, J=7.2 \mathrm{~Hz}, 1 \mathrm{H}), 8.07(\mathrm{~d}, J=7.2 \mathrm{~Hz}, 2 \mathrm{H}) ;{ }^{13} \mathrm{C} \mathrm{NMR}(100 \mathrm{MHz}$, $\left.\mathrm{CDCl}_{3}\right) \delta 14.00,47.85,49.91,62.30,73.21,77.06,128.76,129.16,129.48,130.95$, 134.23, 136.76, 168.89, 171.40, 198.27; IR (neat) v 3446, 1773, 1732, 1681; HRMS $\left(\mathrm{CI} /[\mathrm{M}+1]^{+}\right)$Calcd. for: $\mathrm{C}_{17} \mathrm{H}_{16} \mathrm{BrO}_{6}: 395.0130$. Found: 395.0125 .

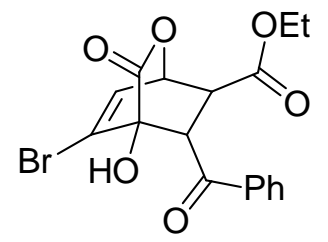

endo-5eA

endo-5eA (minor diastereomers) obtained from the same reaction was isolated as a colorless oil $(4.8 \mathrm{mg}, 4 \%$ yield) in $7 \%$ ee [determined by HPLC, Chiralcel OJ, hexanes: isopropanol $=70: 30$, $0.8 \mathrm{~mL} / \mathrm{min}, \lambda=220 \mathrm{~nm}, \mathrm{t}$ (major) $=39.5 \mathrm{~min}, \mathrm{t}($ minor $)=20.9 \mathrm{~min}]$. $[\alpha]^{\mathrm{D}}{ }_{25}=+2.6\left(c \quad 0.19, \mathrm{CHCl}_{3}\right) ;{ }^{1} \mathrm{H} \mathrm{NMR}\left(400 \mathrm{MHz}, \mathrm{CDCl}_{3}\right) \delta 1.28(\mathrm{t}$, $J=7.2 \mathrm{~Hz}, 3 \mathrm{H}), 3.43(\mathrm{~d}, J=4.8 \mathrm{~Hz}, 1 \mathrm{H}), 3.87(\mathrm{~s}, 1 \mathrm{H}), 4.25(\mathrm{q}, J=7.2 \mathrm{~Hz}, 2 \mathrm{H}), 4.60$ (d, $\mathrm{J}=4.8 \mathrm{~Hz}, 1 \mathrm{H}), 5.69(\mathrm{~d}, J=6.0 \mathrm{~Hz}, 1 \mathrm{H}), 6.82(\mathrm{~d}, J=6.0 \mathrm{~Hz}, 1 \mathrm{H}), 7.50(\mathrm{t}, J=8.0 \mathrm{~Hz}$, 2H), $7.62(\mathrm{t}, J=7.2 \mathrm{~Hz}, 1 \mathrm{H}), 8.04(\mathrm{~d}, J=8.4 \mathrm{~Hz}, 2 \mathrm{H}) ;{ }^{13} \mathrm{C} \mathrm{NMR}\left(100 \mathrm{MHz}, \mathrm{CDCl}_{3}\right) \delta$ 
$14.06,44.55,49.62,62.50,75.95,76.79,128.63,128.67,128.83,129.28,134.05,136.91$, 168.83, 171.69, 196.16; IR (neat) v 3444, 1769, 1732, 1679; HRMS (CI/[M+1] $\left.]^{+}\right)$Calcd. for: $\mathrm{C}_{17} \mathrm{H}_{16} \mathrm{BrO}_{6}: 395.0130$. Found: 395.0137 .

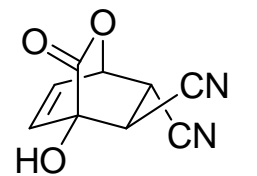

exo-5aE

\section{4-hydroxy-3-oxo-2-oxabicyclo[2.2.2]oct-5-ene-7,8-dicarbonitrile (5aE)}

QD-2-catalyzed ( $8.9 \mathrm{mg}, 0.015 \mathrm{mmol}, 5 \mathrm{~mol} \%)$ reaction of $3 \mathbf{a}(33.6 \mathrm{mg}$, $0.3 \mathrm{mmol})$ and $5 \mathbf{E}(46.8 \mathrm{mg}, 0.6 \mathrm{mmol}, 2.0$ equiv.) in TBME $(3.0 \mathrm{~mL})$ was run at $-20{ }^{\circ} \mathrm{C}$ for $36 \mathrm{~h}$ to furnish the crude product \{exo:endo $>97: 3$, determined by integration of one set of ${ }^{1} \mathrm{H}$ NMR signals $\left[\delta_{\text {major }}=5.61-5.63 \mathrm{ppm}(\mathrm{m})\right.$, $\left.\left.\delta_{\text {minor }}=5.53-5.55 \mathrm{ppm},(\mathrm{m})\right]\right\}$. The crude product was purified by flash chromatography (silica gel, hexanes/acetone $=2 / 1)$ to give adduct exo-5aE as white solid $(48.4 \mathrm{mg}, 85 \%$ yield, $\mathrm{dr}>97: 3$ ) in $92 \%$ ee (major diastereomer) [determined by HPLC, Chiralpak AS, hexanes: isopropanol $=60: 40,0.6 \mathrm{~mL} / \mathrm{min}, \lambda=220 \mathrm{~nm}, \mathrm{t}$ (major) $=26.0 \mathrm{~min}, \mathrm{t}($ minor $)=$ $30.4 \mathrm{~min}] .[\alpha]^{\mathrm{D}}{ }_{25}=+109.8(c 0.50, \mathrm{MeOH}) ;{ }^{1} \mathrm{H} \mathrm{NMR}(400 \mathrm{MHz}, \mathrm{MeOH}) \delta 3.61(\mathrm{~d}, J=$ $5.6 \mathrm{~Hz}, 1 \mathrm{H}), 4.11(\mathrm{dd}, J=3.6,5.2 \mathrm{~Hz}, 1 \mathrm{H}), 5.61-5.63(\mathrm{~m}, 1 \mathrm{H}), 6.68(\mathrm{dd}, J=2.4,8.4 \mathrm{~Hz}$, $1 \mathrm{H}), 6.73(\mathrm{dd}, J=4.8,8.0 \mathrm{~Hz}, 1 \mathrm{H}) ;{ }^{13} \mathrm{C} \mathrm{NMR}(100 \mathrm{MHz}, \mathrm{MeOH}) \delta 35.73,36.97,73.02$, 75.71, 117.33, 117.41, 131.29, 140.02, 171.05; IR (neat) $\vee$ 3419, 2253, 1771; HRMS $\left(\mathrm{CI} /[\mathrm{M}+\mathrm{H}]^{+}\right)$Calcd. for: $\mathrm{C}_{9} \mathrm{H}_{7} \mathrm{O}_{3} \mathrm{~N}_{2}: 191.0457$. Found: 191.0457 .

Q-2-catalyzed reaction under the same conditions for $72 \mathrm{~h}$ furnished crude ent-5aE (exo:endo > 97:3). After chromatographic purification, ent-exo-5aE was obtained in 87\% yield ( $49.7 \mathrm{mg}, 85 \%$ ee).

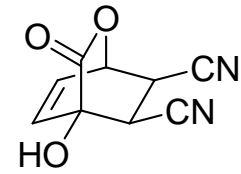

exo-5aF

\section{4-hydroxy-3-oxo-2-oxabicyclo[2.2.2]oct-5-ene-7,8-dicarbonitrile (5aF)} QD-2-catalyzed ( $8.9 \mathrm{mg}, 0.015 \mathrm{mmol}, 5 \mathrm{~mol} \%)$ reaction of $3 \mathbf{a}(33.6 \mathrm{mg}$, $0.3 \mathrm{mmol}$ ) and $\mathbf{4 F}$ (46.8 $\mathrm{mg}, 0.6 \mathrm{mmol}, 2.0$ equiv.) in TBME $(3.0 \mathrm{~mL})$ was run at $-20{ }^{\circ} \mathrm{C}$ for $20 \mathrm{~h}$ to furnish the crude product \{exo:endo $=93: 7$, determined by integration of one set of ${ }^{1} \mathrm{H}$ NMR signals $\left[\delta_{\text {major }}=3.82 \mathrm{ppm}(\mathrm{d}), \delta_{\text {minor }}=\right.$ $4.35 \mathrm{ppm}(\mathrm{dd})]\}$. The crude product was purified by flash chromatography (silica gel, hexanes/acetone $=1 / 2$ ) to give pure exo-5aF (major diastereomers) as a white solid (46.0 $\mathrm{mg}, 81 \%$ yield) in $97 \%$ ee [determined by HPLC, Chiralpak AS, hexanes: isopropanol = 
50:50, $1.0 \mathrm{~mL} / \mathrm{min}, \lambda=220 \mathrm{~nm}, \mathrm{t}$ (major) $=18.3 \mathrm{~min}, \mathrm{t}($ minor $)=16.2 \mathrm{~min}] .[\alpha]_{25}^{\mathrm{D}}=-$ 47.4 (c 0.50, acetone); ${ }^{1} \mathrm{H}$ NMR (400 MHz, $\mathrm{d}_{6}$-acetone) $\delta 3.82(\mathrm{~d}, J=10.8 \mathrm{~Hz}, 1 \mathrm{H}), 4.02$ (d, $J=10.8 \mathrm{~Hz}, 1 \mathrm{H}), 5.71(\mathrm{~d}, J=4.8 \mathrm{~Hz}, 1 \mathrm{H}), 6.46(\mathrm{~s}, 1 \mathrm{H}), 6.61(\mathrm{dd}, J=1.2,8.0 \mathrm{~Hz}$, $1 \mathrm{H}), 6.70(\mathrm{dd}, J=3.6,8.0 \mathrm{~Hz}, 1 \mathrm{H}) ;{ }^{13} \mathrm{C} \mathrm{NMR}\left(100 \mathrm{MHz}, \mathrm{d}_{6}\right.$-acetone) $\delta 34.22,34.62$, 73.45, 74.62, 115.72, 116.52, 131.20, 137.94, 169.97; IR (neat) $\vee 3329,1770$; HRMS $\left(\mathrm{CI} /[\mathrm{M}+\mathrm{H}]^{+}\right)$Calcd. for: $\mathrm{C}_{9} \mathrm{H}_{7} \mathrm{O}_{3} \mathrm{~N}_{2}: 191.0457$. Found: 191.0454 .

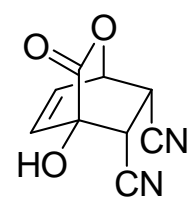
endo-5aF (minor diastereomers) obtained from the same reaction was isolated as a white solid (3.4 mg, 6\% yield) in 33\% ee [determined by HPLC, Chiralpak AS, hexanes: isopropanol $=50: 50,1.0 \mathrm{~mL} / \mathrm{min}, \lambda=220 \mathrm{~nm}, \mathrm{t}$ endo-5aF $($ major $)=14.4 \mathrm{~min}, \mathrm{t}($ minor $)=11.0 \mathrm{~min}] .[\alpha]_{25}^{\mathrm{D}}=-20.0($ c 0.05 , acetone $)$;

${ }^{1} \mathrm{H}$ NMR (400 MHz, d ${ }_{6}$-acetone) $\delta 3.96(\mathrm{~d}, J=9.6 \mathrm{~Hz}, 1 \mathrm{H}), 4.34(\mathrm{dd}, J=3.2,9.2 \mathrm{~Hz}$, $1 \mathrm{H}), 5.77(\mathrm{~m}, 1 \mathrm{H}), 6.38(\mathrm{~s}, 1 \mathrm{H}), 6.78(\mathrm{~d}, J=8.0 \mathrm{~Hz}, 1 \mathrm{H}), 6.89(\mathrm{dd}, J=4.8,8.0 \mathrm{~Hz}, 1 \mathrm{H})$; ${ }^{13} \mathrm{C}$ NMR $\left(100 \mathrm{MHz}, \mathrm{CD}_{3} \mathrm{OD}\right) \delta 34.49,35.42,71.73,74.41,115.68,116.04,130.80$, 138.05, 170.07; IR (neat) $\vee 3309$, 1759; HRMS (CI/[M+H] $]^{+}$Calcd. for: $\mathrm{C}_{9} \mathrm{H}_{7} \mathrm{O}_{3} \mathrm{~N}_{2}$ : 191.0457. Found: 191.0452.].

Q-2-catalyzed reaction under the same conditions for $20 \mathrm{~h}$ furnished crude ent-5aF (exo:endo $=89: 11)$. After chromatographic purification, ent-exo-5aF was obtained in $89 \%$ yield (52.2 $\mathrm{mg},>98 \%$ ee), and ent-endo-5aF was obtained in $11 \%$ yield $(6.1 \mathrm{mg}$, $37 \%$ ee).

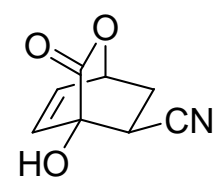

exo-5aG

\section{4-hydroxy-3-oxo-2-oxabicyclo[2.2.2]oct-5-ene-8-carbonitrile (5aG)}

QD-2-catalyzed (8.9 mg, $0.015 \mathrm{mmol}, 5 \mathrm{~mol} \%)$ reaction of 3a $(33.6 \mathrm{mg}$, $0.3 \mathrm{mmol})$ and $4 \mathrm{G}(80 \mu \mathrm{L}, 1.2 \mathrm{mmol}, 4.0$ equiv.) in TBME (3.0 mL) was run at room temperature for $48 \mathrm{~h}$ to furnish the crude product \{exo:endo= $96: 4$, determined by integration of one set of ${ }^{1} \mathrm{H}$ NMR signals $\left[\delta_{\text {major }}=2.60(\mathrm{td}), \delta_{\text {minor }}=\right.$ $2.76 \mathrm{ppm}$ (ddd)]\}. The crude product was purified by flash chromatography (silica gel, hexanes/acetone $=2 / 1$ ) to give pure exo-5aG (major diastereomers) as a white solid (45.0 $\mathrm{mg}, 91 \%$ yield) in $94 \%$ ee [determined by HPLC, Chiralcel OJ, hexanes: isopropanol $=$ $60: 40,0.85 \mathrm{~mL} / \mathrm{min}, \lambda=220 \mathrm{~nm}, \mathrm{t}$ (major) $=22.6 \mathrm{~min}, \mathrm{t}($ minor $)=18.3 \mathrm{~min}] .[\alpha]_{25}^{\mathrm{D}}=$ - 
$19.4\left(\right.$ c 0.50, $\left.\mathrm{CHCl}_{3}\right) ;{ }^{1} \mathrm{H}$ NMR (400 MHz, $\left.\mathrm{CDCl}_{3}\right) \delta 2.30(\mathrm{dd}, J=11.6,12.8 \mathrm{~Hz}, 1 \mathrm{H})$, $2.60(\mathrm{td}, J=4.8,14.0 \mathrm{~Hz}, 1 \mathrm{H}), 3.03(\mathrm{dd}, J=5.2,10.8 \mathrm{~Hz}, 1 \mathrm{H}), 4.11(\mathrm{~s}, 1 \mathrm{H}), 5.36-5.37$ $(\mathrm{m}, 1 \mathrm{H}), 6.52-6.56(\mathrm{~m}, 2 \mathrm{H}) ;{ }^{13} \mathrm{C}$ NMR $\left(100 \mathrm{MHz}, \mathrm{CDCl}_{3}\right) \delta 30.78,32.05,72.72,74.53$, 117.63, 131.07, 136.27, 171.66; IR (neat) v 3354, 2244, 1756; HRMS (CI/[M+H] $]^{+}$Calcd. for: $\mathrm{C}_{8} \mathrm{H}_{8} \mathrm{O}_{3} \mathrm{~N}: 166.0504$. Found: 166.0505 .

Q-2-catalyzed reaction under the same conditions for $72 \mathrm{~h}$ furnished crude ent-5aG (exo:endo $=93: 7)$. After chromatographic purification, ent-exo-5aG was obtained in 87\% yield (43.3 mg, 93\% ee).

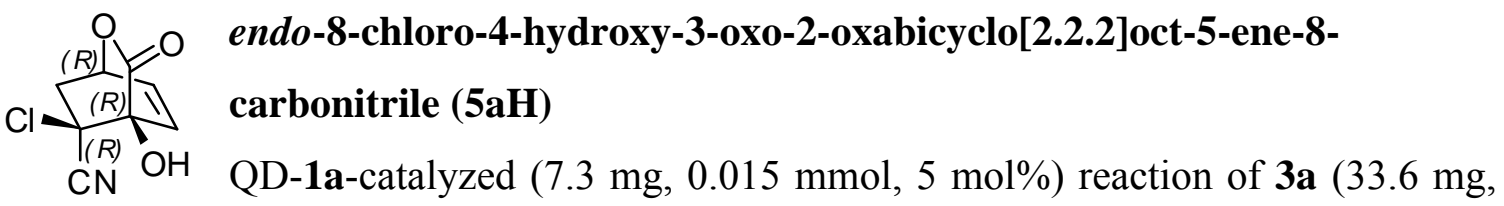

$\mathbf{5 a H} \quad 0.3 \mathrm{mmol})$ and $\mathbf{4 H}(96 \mu \mathrm{L}, 1.2 \mathrm{mmol}, 4.0$ equiv.) in diethyl ether (3.0 mL) was run at room temperature for $36 \mathrm{~h}$ to furnish the crude product endo:exo $=83: 17$, determined by integration of one set of ${ }^{1} \mathrm{H}$ NMR signals $\left[\delta_{\text {major }}=3.04 \mathrm{ppm}(\mathrm{dd}), \delta_{\text {minor }}=\right.$ $3.33 \mathrm{ppm}(\mathrm{dd})]\}$. The crude product was purified by flash chromatography (silica gel, hexanes/acetone $=3 / 1)$ to give the reaction adducts as a white solid $(54.0 \mathrm{mg}, 90 \%$ yield, $\mathrm{dr}=83: 17)$ in $85 \%$ ee (5aH, major diastereomer) [determined by HPLC, Chiralpak AS, hexanes: isopropanol $=60: 40,0.4 \mathrm{~mL} / \mathrm{min}, \lambda=220 \mathrm{~nm}, \mathrm{t}$ (major) $=22.9 \mathrm{~min}, \mathrm{t}($ minor $)=$ $31.6 \mathrm{~min}] .[\alpha]_{25}^{\mathrm{D}}=+5.4\left(\right.$ c $\left.0.50, \mathrm{CHCl}_{3}\right) ;{ }^{1} \mathrm{H} \mathrm{NMR}\left(400 \mathrm{MHz}, \mathrm{CDCl}_{3}\right)$ of the major diastereomer, $\delta 2.95(\mathrm{dd}, J=2.0,15.6 \mathrm{~Hz}, 1 \mathrm{H}), 3.02(\mathrm{dd}, J=3.2,14.8 \mathrm{~Hz}, 1 \mathrm{H}), 4.22(\mathrm{~s}$, $1 \mathrm{H}), 5.36-5.39(\mathrm{~m}, 1 \mathrm{H}), 6.62(\mathrm{dd}, J=1.6,8.4 \mathrm{~Hz}, 1 \mathrm{H}), 6.76(\mathrm{dd}, J=5.6,8.0 \mathrm{~Hz}, 1 \mathrm{H})$; ${ }^{13} \mathrm{C}$ NMR $\left(100 \mathrm{MHz}, \mathrm{CDCl}_{3}\right)$ of the major diastereomer, $\delta 45.62,55.99,72.19,78.95$, 116.45, 133.30, 133.53, 169.06; IR (neat) v 3444, 2247, 1767; HRMS (CI/[M+H] $]^{+}$Calcd. for: $\mathrm{C}_{8} \mathrm{H}_{7} \mathrm{O}_{3} \mathrm{NCl}: 200.0114$. Found: 200.0112. The single crystal of $(R, R, R)-5$ aH was obtained by recrystalization from $\mathrm{CHCl}_{3}$.

Q-1a-catalyzed reaction under the same conditions for $96 \mathrm{~h}$ furnished ent-adducts in $85 \%$ yield (51.2 mg, exo:endo $=22: 78,75 \%$ ee for ent-5aH) 


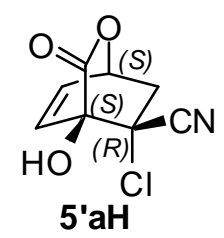

exo-8-chloro-4-hydroxy-3-oxo-2-oxabicyclo[2.2.2] ]oct-5-ene-8carbonitrile (5’aH)

QD-2-catalyzed (8.9 mg, $0.015 \mathrm{mmol}, 5 \mathrm{~mol} \%)$ reaction of 3a (33.6 $\mathrm{mg}$, $0.3 \mathrm{mmol})$ and $\mathbf{4 H}(96 \mu \mathrm{L}, 1.2 \mathrm{mmol}, 4.0$ equiv.) in THF (3.0 mL) was run at room temperature for $48 \mathrm{~h}$ to furnish the crude product endo:exo $=7: 93$, determined by integration of one set of ${ }^{1} \mathrm{H}$ NMR signals $\left[\delta_{\text {major }}=3.33 \mathrm{ppm}(\mathrm{dd}), \delta_{\text {minor }}=3.04 \mathrm{ppm}\right.$ $(\mathrm{dd})]\}$. The crude product was purified by flash chromatography (silica gel, hexanes/acetone $=3 / 1)$ to give reaction adducts as a white solid $(54.5 \mathrm{mg}, 91 \%$ yield, $\mathrm{dr}$ $=7: 93$ ) in $89 \%$ ee (5'aH, major diastereomer) [determined by HPLC, Chiralpak AS, hexanes: isopropanol $=60: 40,0.4 \mathrm{~mL} / \mathrm{min}, \lambda=220 \mathrm{~nm}, \mathrm{t}$ (major) $=33.2 \mathrm{~min}, \mathrm{t}$ (minor) $=$ $21.5 \mathrm{~min}] .[\alpha]^{\mathrm{D}}{ }_{25}=+12.1\left(c 0.43, \mathrm{CHCl}_{3}\right) ;{ }^{1} \mathrm{H} \mathrm{NMR}\left(400 \mathrm{MHz}, \mathrm{CDCl}_{3}\right)$ of the major diastereomer, $\delta 2.56(\mathrm{~d}, J=15.6 \mathrm{~Hz}, 1 \mathrm{H}), 3.33(\mathrm{dd}, J=3.2,15.2 \mathrm{~Hz}, 1 \mathrm{H}), 4.25(\mathrm{~s}, 1 \mathrm{H})$, 5.37-5.39 (m, 1H), $6.48(\mathrm{~d}, J=8.0 \mathrm{~Hz}, 1 \mathrm{H}), 6.66(\mathrm{dd}, J=5.6,7.6 \mathrm{~Hz}, 1 \mathrm{H}) ;{ }^{13} \mathrm{C} \mathrm{NMR}$ $\left(100 \mathrm{MHz}, \mathrm{CDCl}_{3}\right)$ of the major diastereomer, $\delta 45.53,55.50,71.50,79.07,116.16$, 130.34, 134.21, 169.72; IR (neat) $\vee$ 3363, 1758; HRMS (CI/[M+H] $]^{+}$) Calcd. for: $\mathrm{C}_{8} \mathrm{H}_{7} \mathrm{O}_{3} \mathrm{NCl}$ : 200.0114. Found: 200.0121. The single crystal of (1S, 4S, 5R)-5'aH was obtained by recrystalization from $\mathrm{CHCl}_{3}$.

Q-2-catalyzed reaction under the same condition for $48 \mathrm{~h}$ furnished the reaction adducts in 93\% yield (55.9 mg, exo:endo $=91: 9,85 \%$ ee for ent-5’aH). 


\section{Relative Configuration Assignment of Cycloadducts 5}

4.1 Assignment of the absolute configuration of 5aH and 5'aH by X-ray analysis.
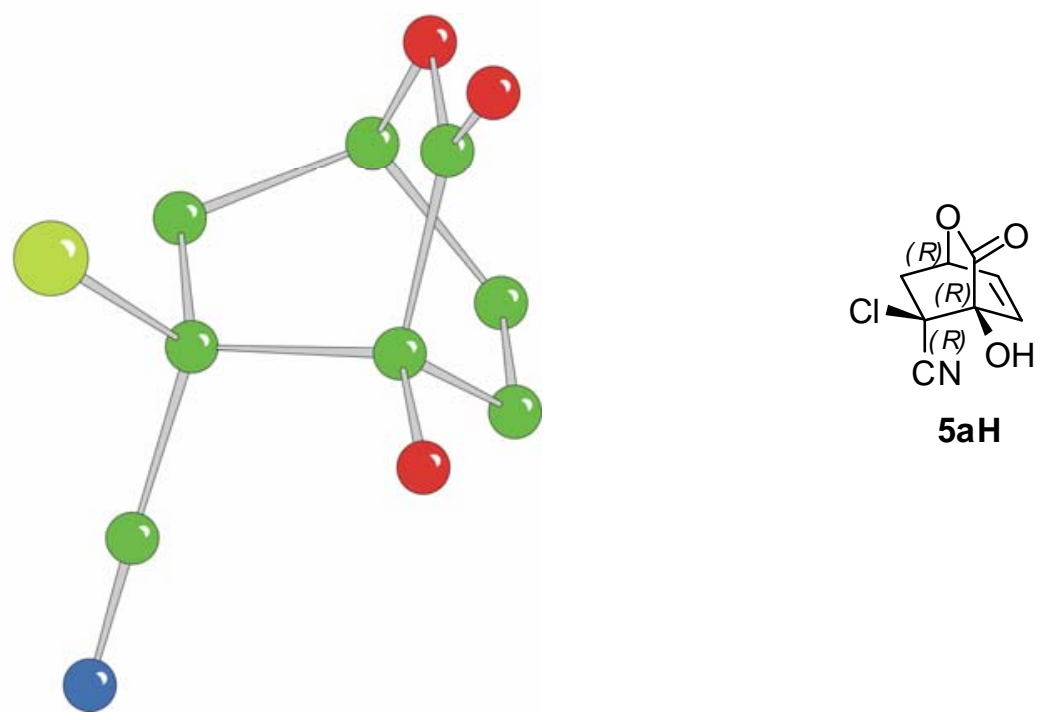

$5 \mathrm{aH}$

S-Figure 3. X-ray structure of $\mathbf{5 a H}$ (hydrogen was omitted for clearance)

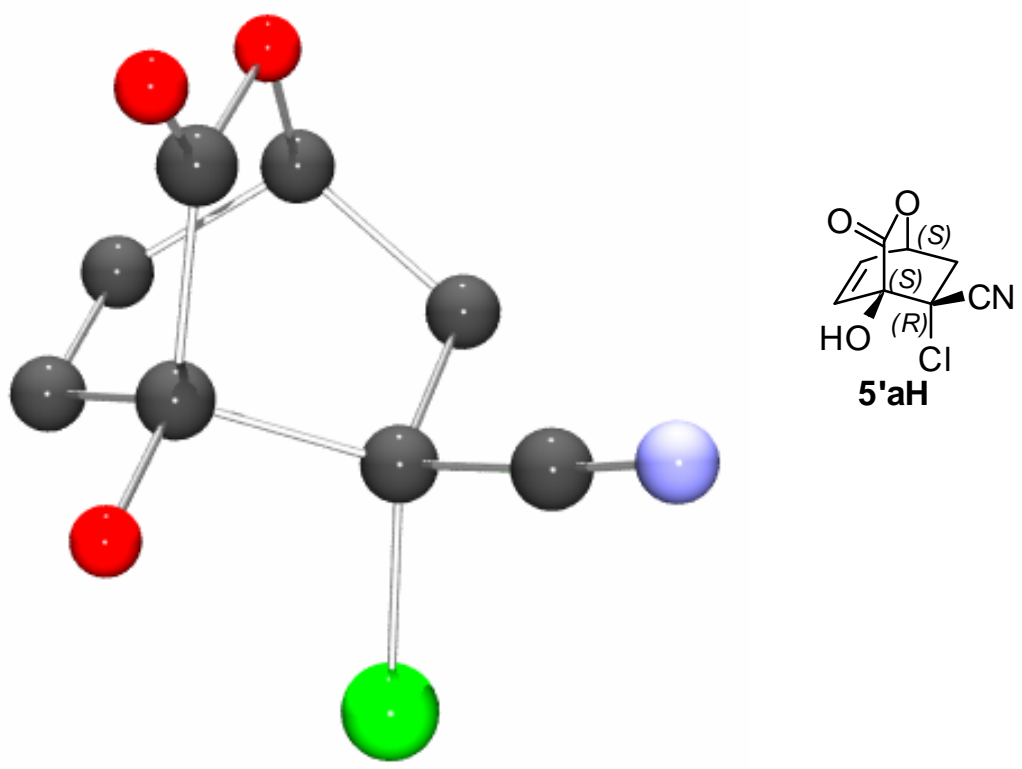

S-Figure 4. X-ray structure of 5’aH (hydrogen was omitted for clearance) 


\subsection{Assignment of the relative configuration of $5 \mathrm{aD}$ by NOE study}

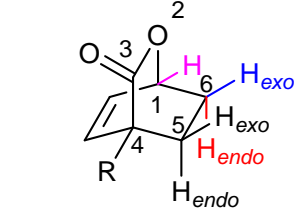

Reported Coupling Constant $\mathrm{H}_{1}-\mathrm{H}_{\text {6endo }}=1.1-1.5 \mathrm{~Hz}$ $\mathrm{H}_{1}-\mathrm{H}_{6 \mathrm{exo}}=2.0-4.5 \mathrm{~Hz}$

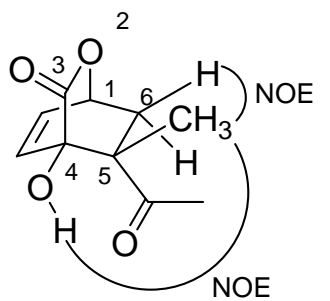

endo-5aD

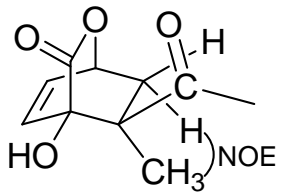

exo-5aD

As described by Ponser ${ }^{12}, \mathrm{H}_{6 e n d o}$ and $\mathrm{H}_{6 \text { exo }}$ could be assigned by their respective coupling constant with $\mathrm{H}_{1}: \mathrm{H}_{1}-\mathrm{H}_{6 e n d o}=1.1-1.5 \mathrm{~Hz}, \mathrm{H}_{1}-\mathrm{H}_{6 e n d o}=2.0-4.5 \mathrm{~Hz}$. After $\mathrm{H}_{6 e n d o}$ and $\mathrm{H}_{6 \text { exo }}$ were assigned by this method, the configuration of $\mathrm{C}_{5}$ relative to $\mathrm{C}_{6}$ was assigned by $\mathrm{NOE}$ between $\mathrm{H}_{6 e n d o}$ or $\mathrm{H}_{6 e x o}$ with the $\mathrm{C}_{5}$-Me as shown above.

\subsection{Assignment of the relative configuration of other cycloadducts 5 by ${ }^{1} \mathrm{H}$ NMR}

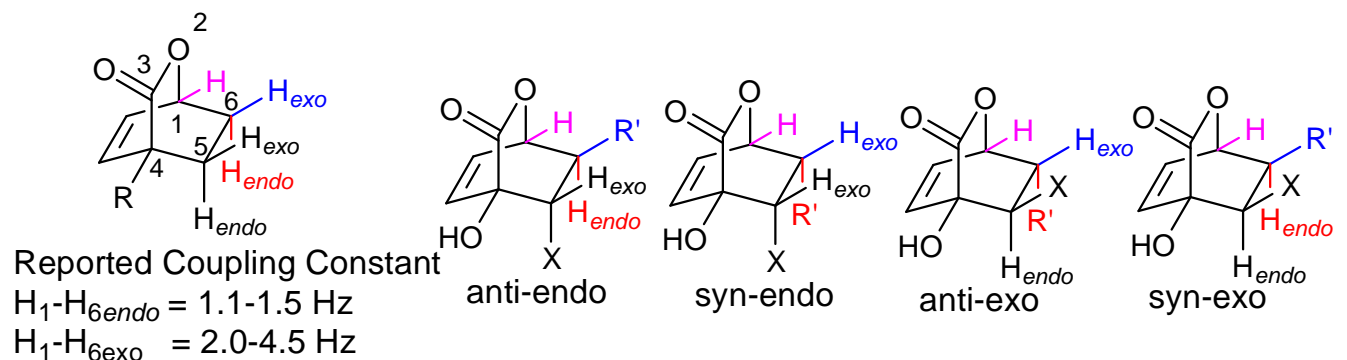

As described by Ponser ${ }^{12}, \mathrm{H}_{6 e n d o}$ and $\mathrm{H}_{6 e x o}$ could be assigned by their respective coupling constant with $\mathrm{H}_{1}: \mathrm{H}_{1}-\mathrm{H}_{6 e n d o}=1.1-1.5 \mathrm{~Hz}, \mathrm{H}_{1}-\mathrm{H}_{6 e n d o}=2.0-4.5 \mathrm{~Hz}$. After $\mathrm{H}_{6 e n d o}$ and/or $\mathrm{H}_{6 e x o}$ were assigned by this method, the configuration of $\mathrm{C}_{5}$ relative to $\mathrm{C}_{6}$ was assigned by $\mathrm{H}_{5}-\mathrm{H}_{6}$ coupling constant (syn, 9-10 Hz; anti, 4-6 Hz). Consequently, the endo or exo configuration could be assigned for the cycloadducts 5 . 
S-Table 1. Determination of the Relative Configurations of the Products in Table 2

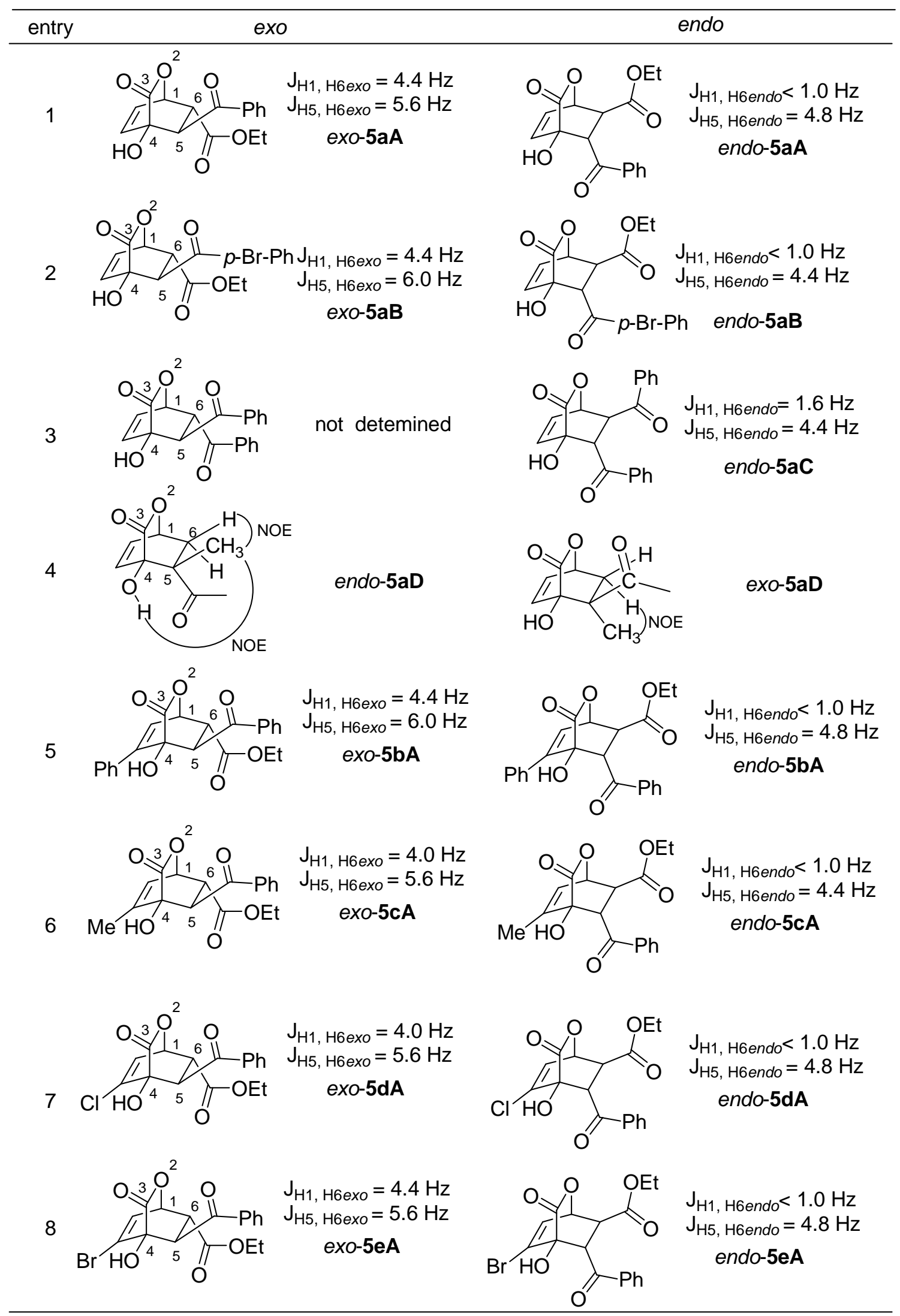


S-Table 2. Determination of the relative configuration of reaction products inTable 3 and Scheme 1

Entry




\section{References}

(1) Mathias, L. J.; Overberger, C, G. Synth. Comm. 1975, 5, 461.

(2) Komiyama, T.; Takaguchi, Y.; Gubaidullin, A. T.; Mamedov, V. A.; Litvinov, I. A.; Tsuboi, S. Tetrahedron 2005, 61, 2541.

(3) Runcie, K. A.; Taylor, R. J. K. Chem. Commun. 2002, 974.

(4) Linstead, R. P.; Whalley, M. J. Chem. Soc. 1952, 4839.

(5) Li, H.; Wang, Y.; Tang, L.; Wu, F.; Liu, X.; Guo, C.; Foxman, B. M.; Deng, L. Angew. Chem., Int. Ed. 2005, 44, 105.

(6) Wang, Y.; Liu, X.; Deng, L. J. Am. Chem. Soc. 2006, 128, 3928.

(7) Wu, F.; Hong, R.; Khan, J.; Liu, X.; Deng, L. Angew. Chem. Int. Ed. 2006, 45, 4301.

(8) Vakulya, B.; Varga, S.; Csámpai, A.; Soós, T. Org Lett. 2005, 7, 1967.

(9) Iwabuchi, Y.; Nakatani, M.; Yokoyama, N.; Hatakeyama, S. J. Am. Chem. Soc. 1999, 121, 10219.

(10) Arad, O.; Morros, J.; Batllori, X.; Teixido', J.; Nonell, S.; Borrell, J. Org. Lett. 2006, 8, 847

(11) Ryu, K.; Cho, Y.-S.; Jung, S.-I.; Cho, C.-G. Org. Lett. 2006, 8, 3343.

(12) Afarinkia, K.; Vinader, V.; Nelson, T. D.; Posner, G. H. Tetrahedron 1992, 48, 9111 and references therein. 
<smiles>O=c1occc(-c2ccccc2)c1O</smiles>
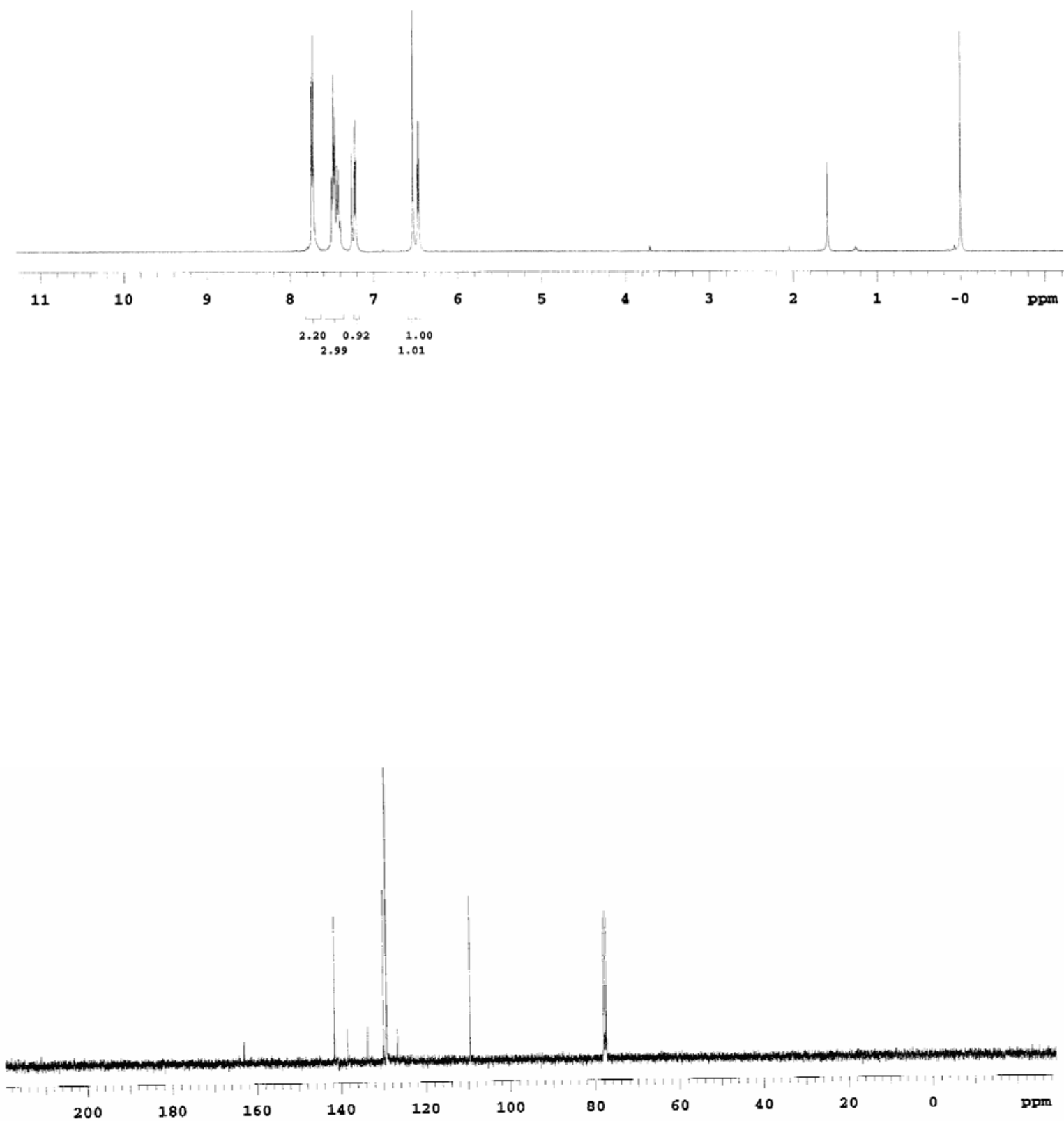

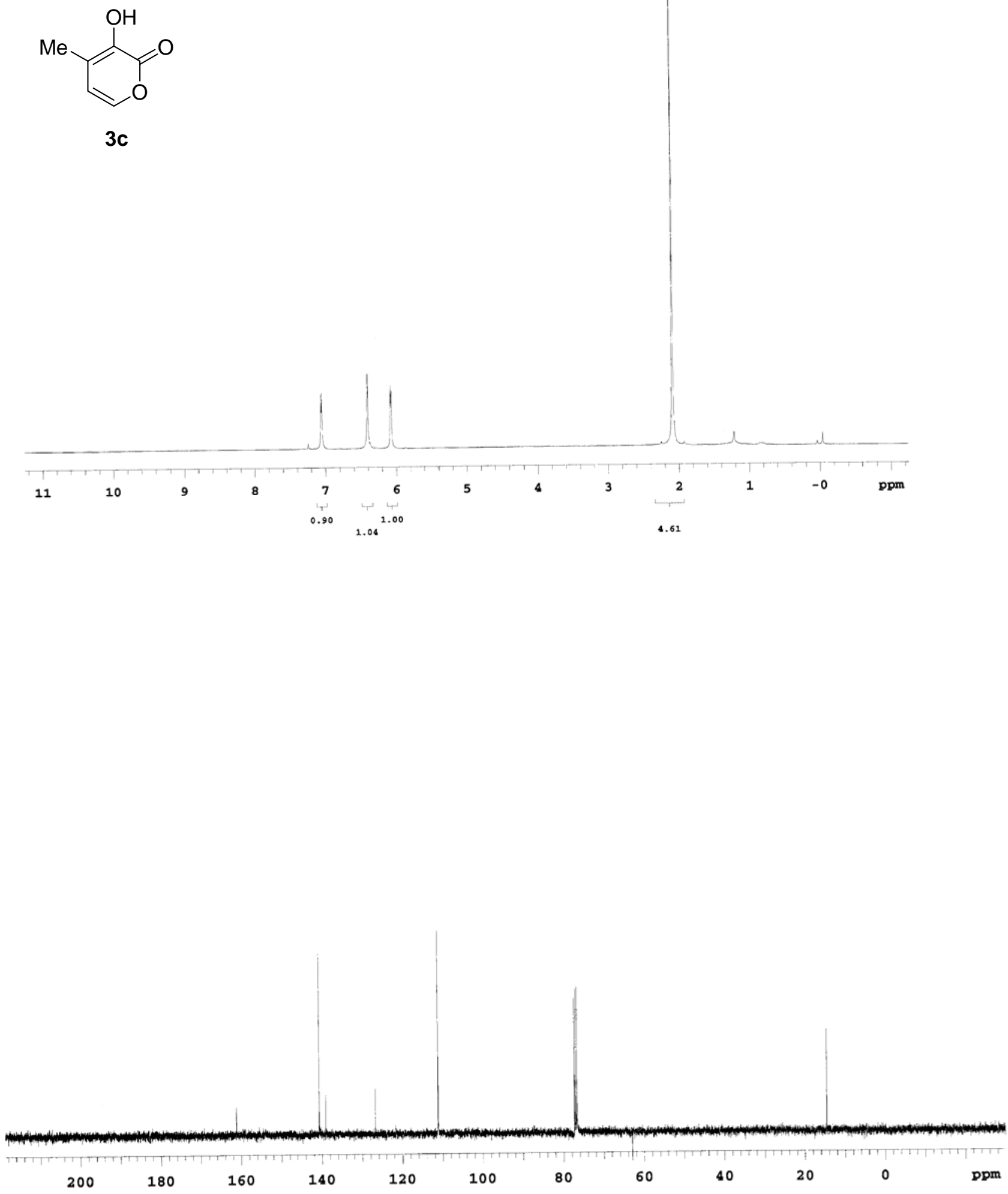

S 22 

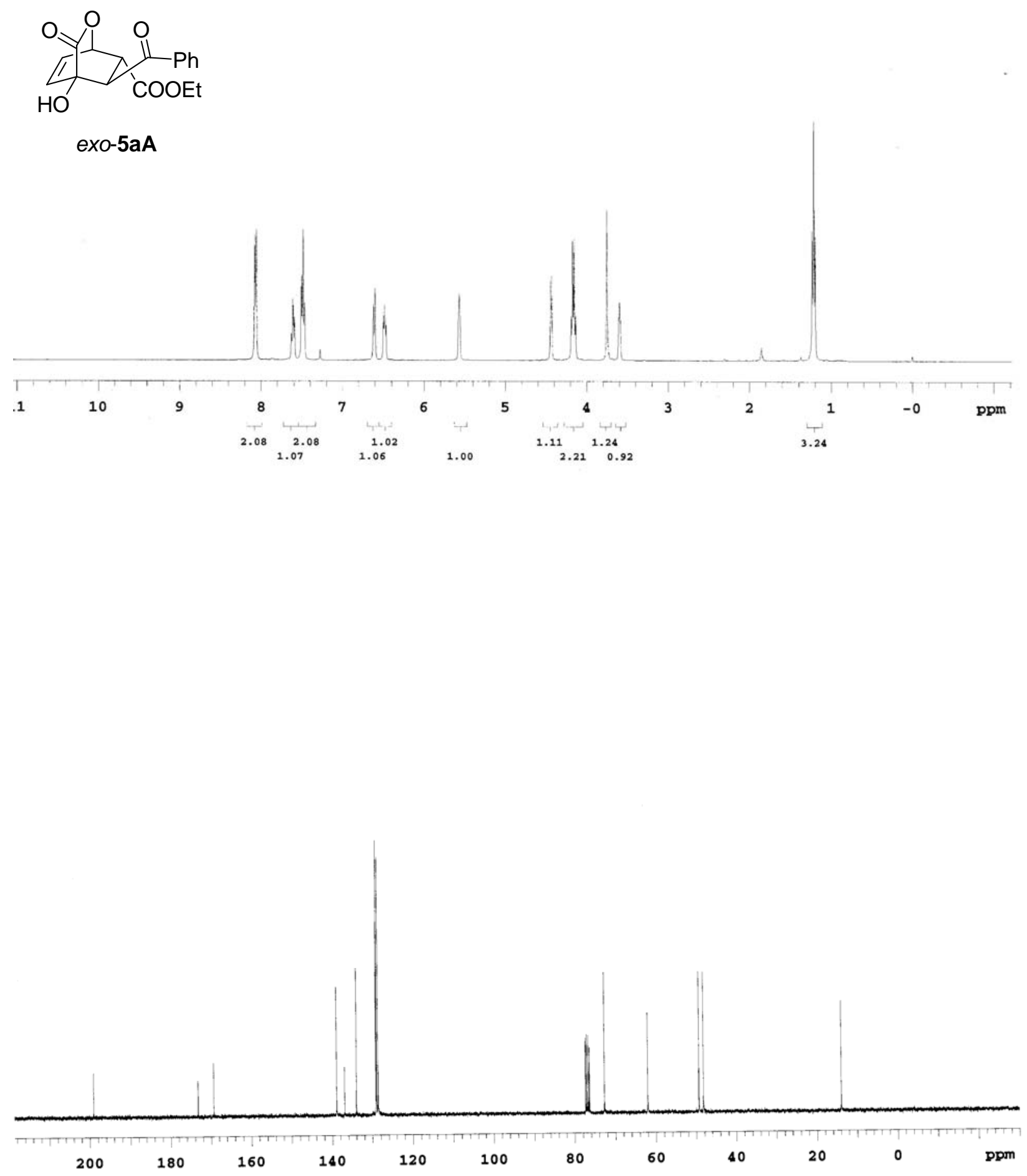


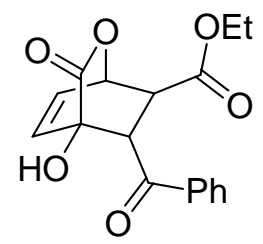

endo-5aA
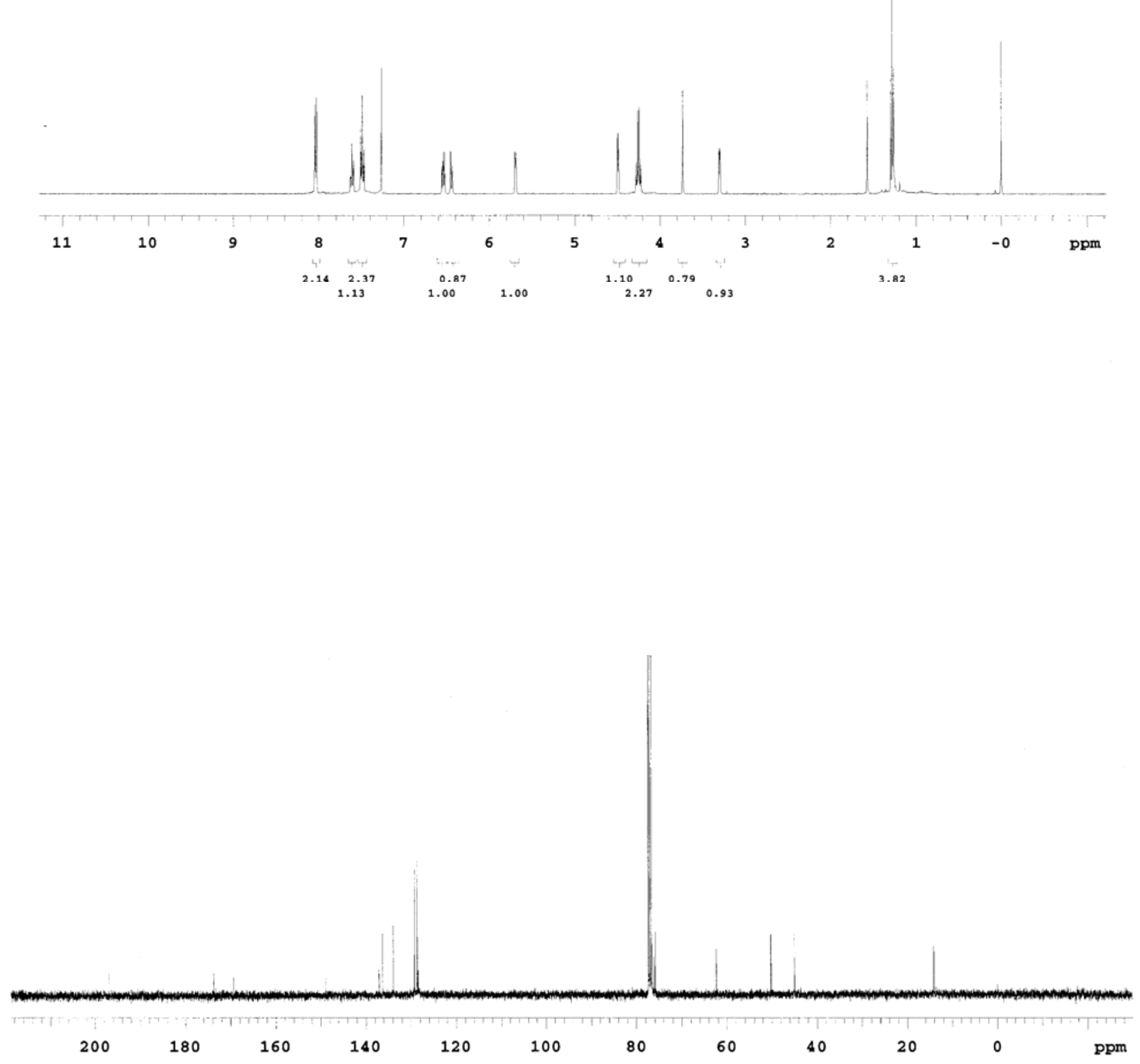
HPLC, $(R, R)$-Whelk-O 1, hexanes: isopropanol $=90: 10,1.0 \mathrm{~mL} / \mathrm{min}, \lambda=220 \mathrm{~nm}$

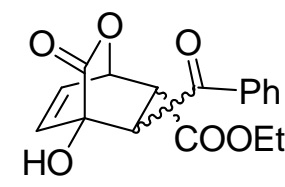

racemic $5 \mathrm{aA}$

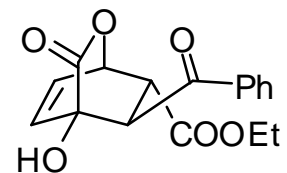

exo-5aA, 94\% ee obtained from QD-1a catalyzed reaction

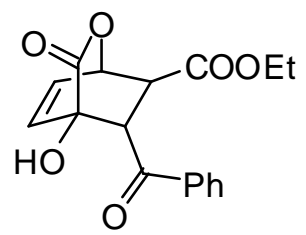

endo-5aA, 54\% ee obtained from QD-1a catalyzed reaction
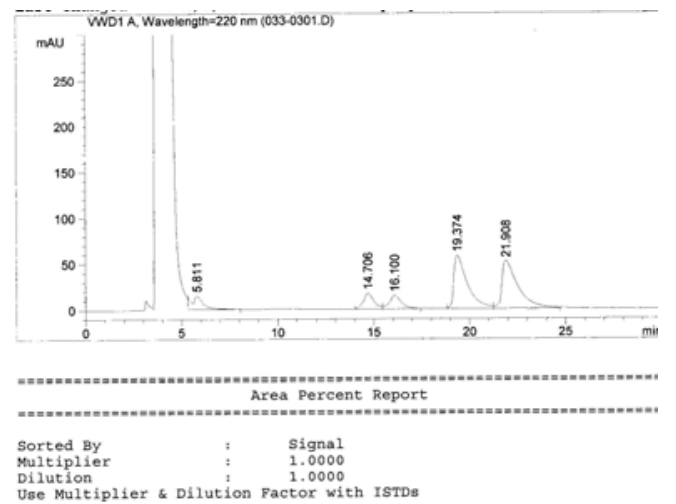

Factor with ISTDE

Signal 1: VWD1 $\mathrm{A}$, wavelength=220 $\mathrm{nm}$
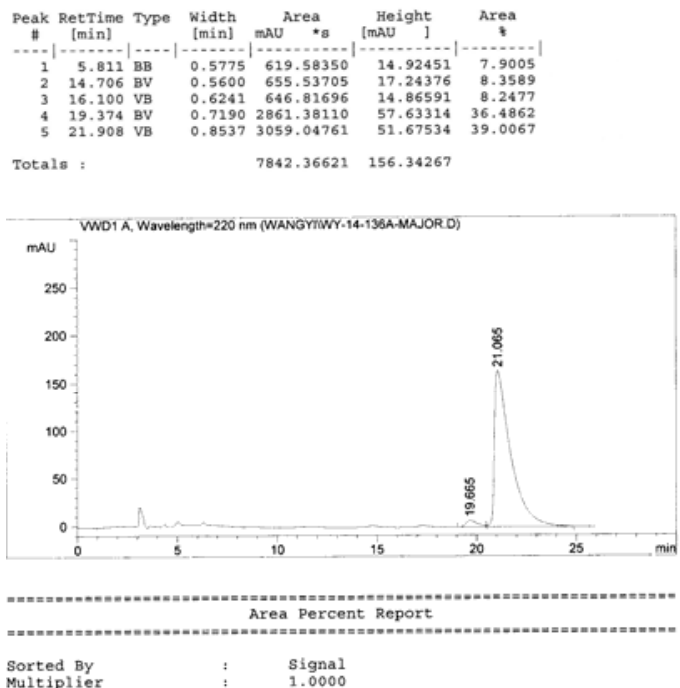

\begin{tabular}{l|l} 
Sorted By & Signal \\
Multiplier & 1.0000
\end{tabular}

1.0000
Sample Amount
Salle

Signal 1: VWD1 A, wavelength $=220 \mathrm{~nm}$

Peak RetTime Type width
$\equiv$ [min]
(min) maU

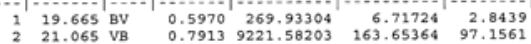

Totals : $\quad 9491.51508 \quad 170.37088$
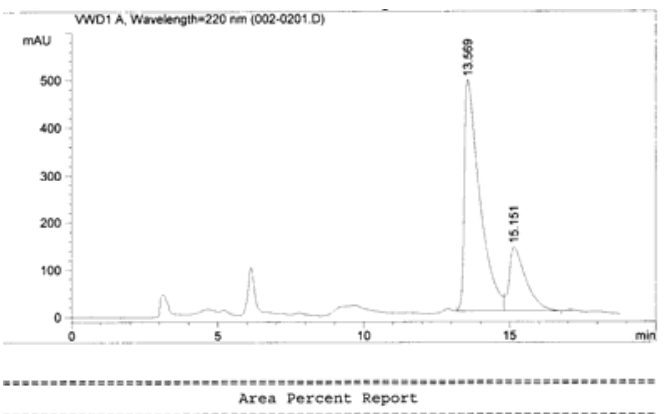

$\begin{array}{lll}\text { Sorted By } & : & \text { Signal } \\ \text { Multiplier } & 1.0000\end{array}$

Dilution 1 il to 1,0000

Signal 1: VWD1 $\mathrm{A}$, wavelength $220 \mathrm{~nm}$

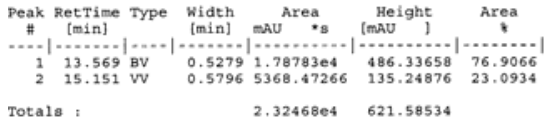


HPLC, $(R, R)$-Whelk-O 1, hexanes: isopropanol $=90: 10,1.0 \mathrm{~mL} / \mathrm{min}, \lambda=220 \mathrm{~nm}$

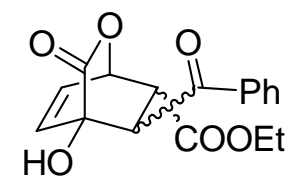

racemic $5 \mathrm{aA}$

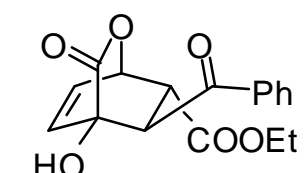

ent-exo-5aA, 87\% ee obtained from Q-1a catalyzed reaction

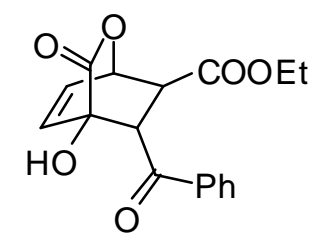

ent-endo-5aA, 23\% ee obtained from Q-1a catalyzed reaction

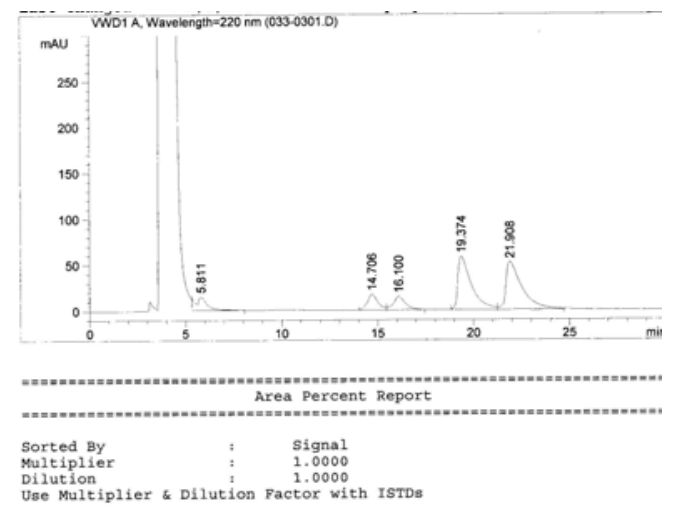

Signal 1: VWD1 $A$, wavelength $=220 \mathrm{~nm}$

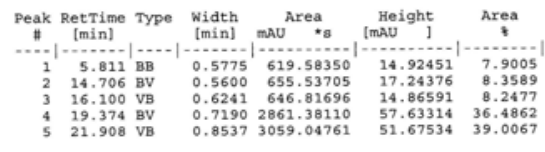

Totals : $\quad 7842.36621 \quad 156.34267$
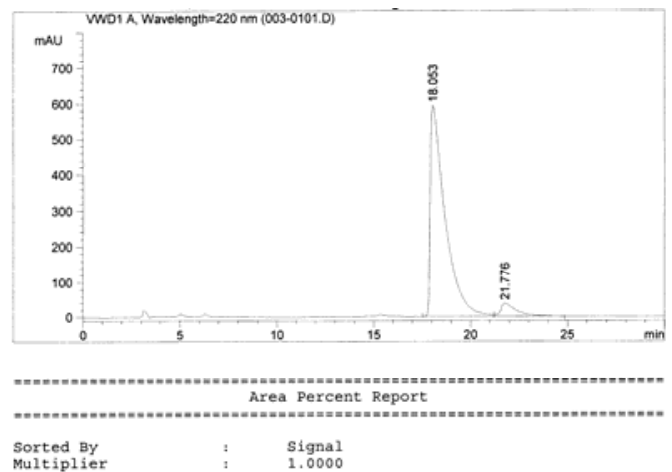

Use Multiplier \& Dilution Pactor with ISTD

Signal 1: VWD1 A, wavelength=220 nm

Peak RetTime Type width Area
In $[\min ]$
$[\min ]$

$\begin{array}{rrrrrr}1 & 18.053 \mathrm{VV} & 0.7558 & 3.15420 \mathrm{e} & 591.76538 & 93.6588 \\ 2 & 21.776 \mathrm{VB} & 0.8165 & 2135.55737 & 37.13686 & 6.3412\end{array}$

Totals : $\quad 3.36776 \mathrm{e} 4 \quad 628.90224$

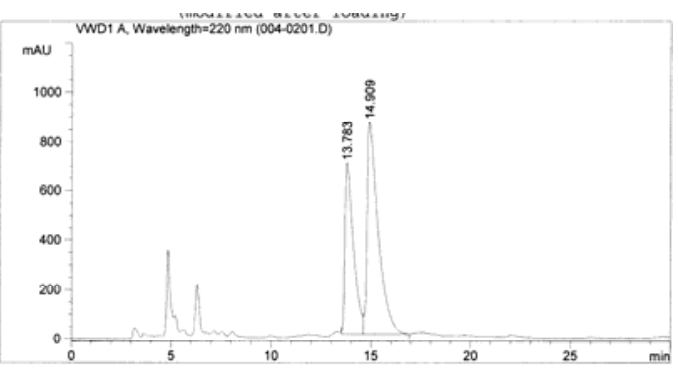

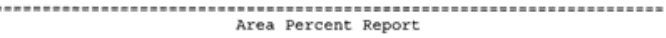

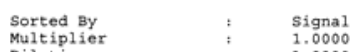

$\begin{array}{lll}\text { Multiplier } & 1.0000 \\ \text { Dilution } & 1.0000\end{array}$

Use Multiplier \& Dilution Factor with ISTDs

Signal 1: VWD1 $A$, wavelength $220 \mathrm{~nm}$

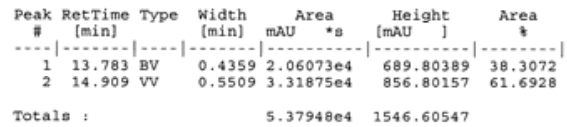




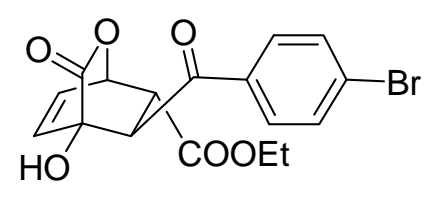

exo-5aB
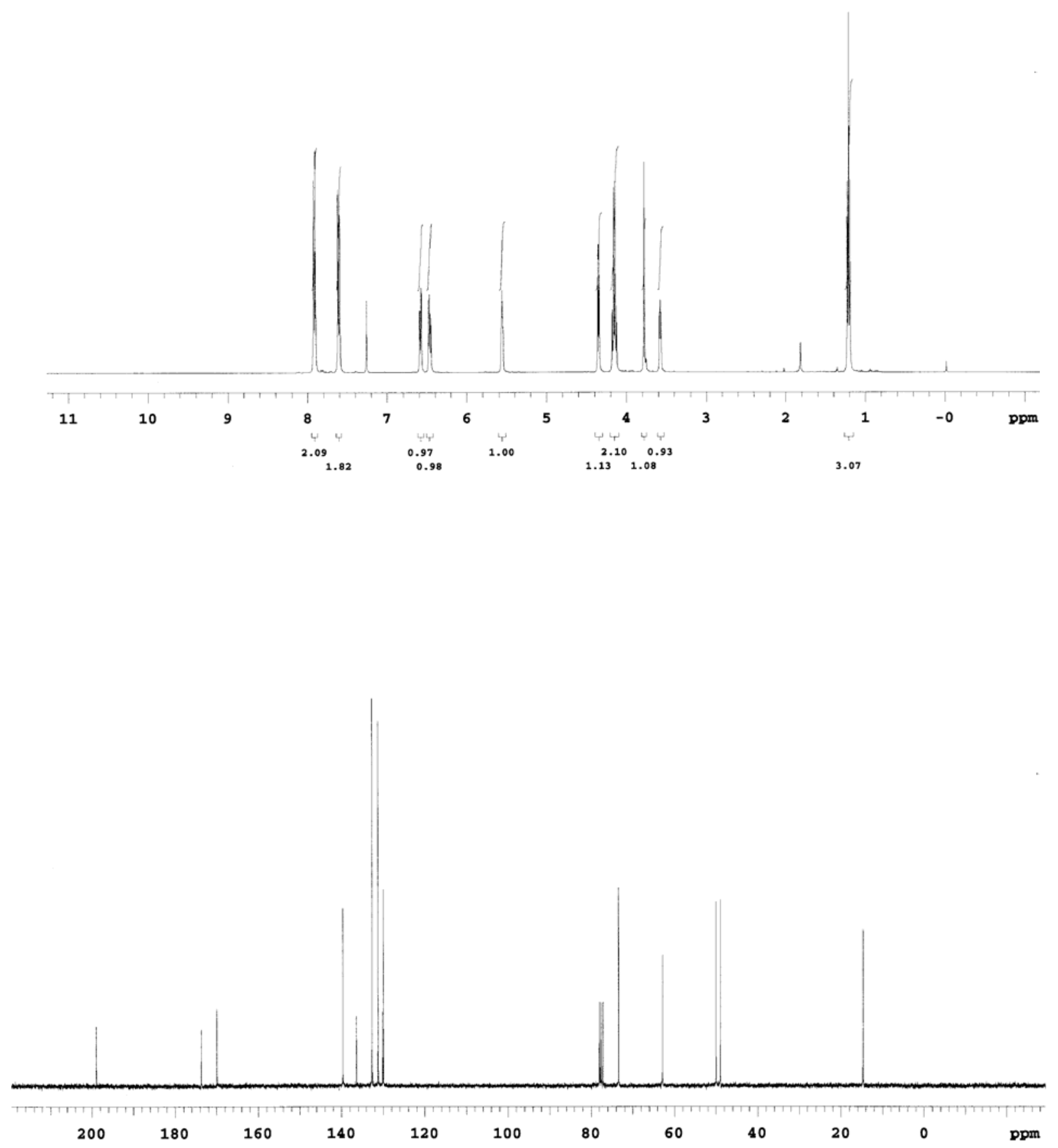

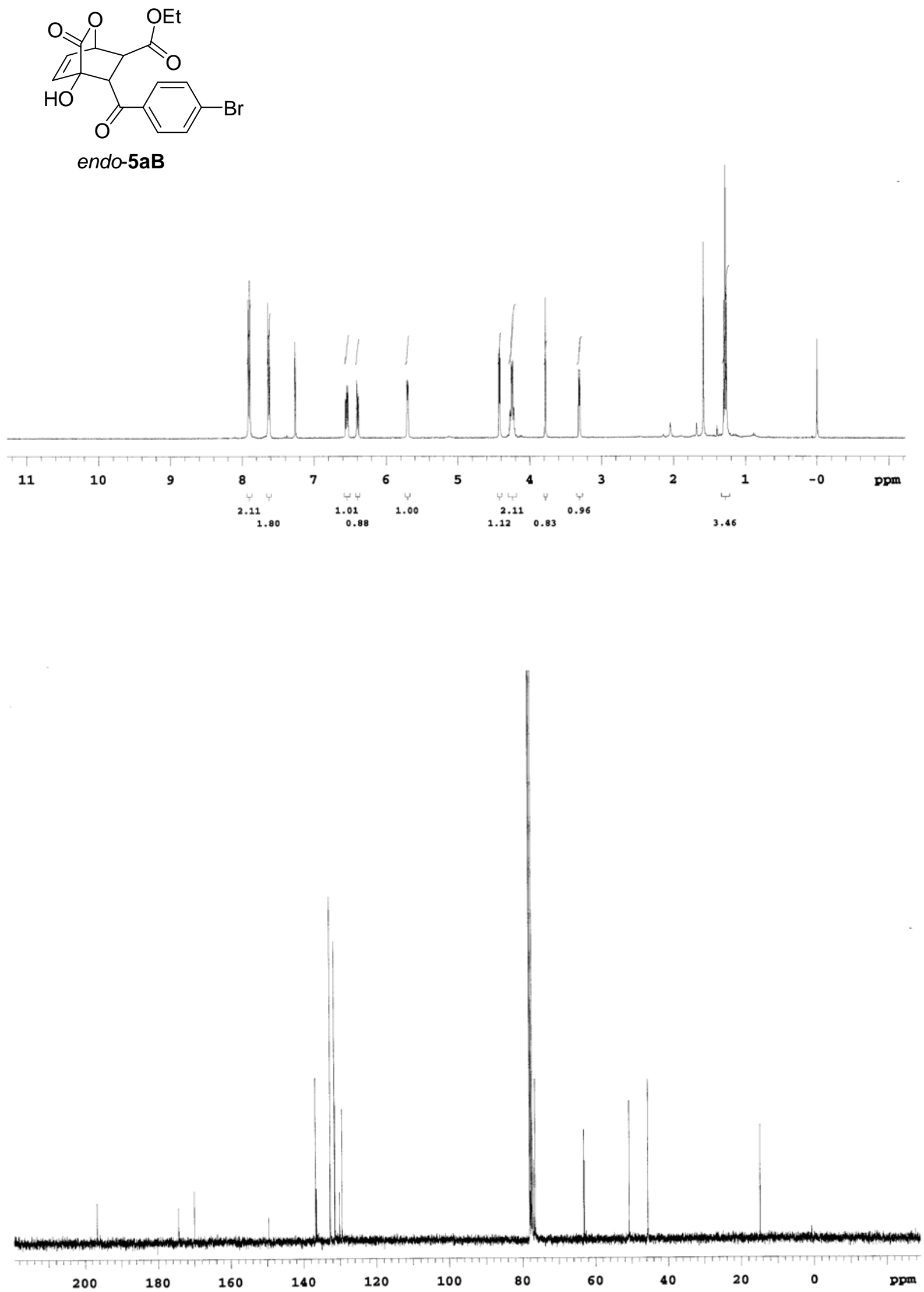

S 28 
HPLC, Chiralpak AD, hexanes: isopropanol $=80: 20,1.0 \mathrm{~mL} / \mathrm{min}, \lambda=220 \mathrm{~nm}$
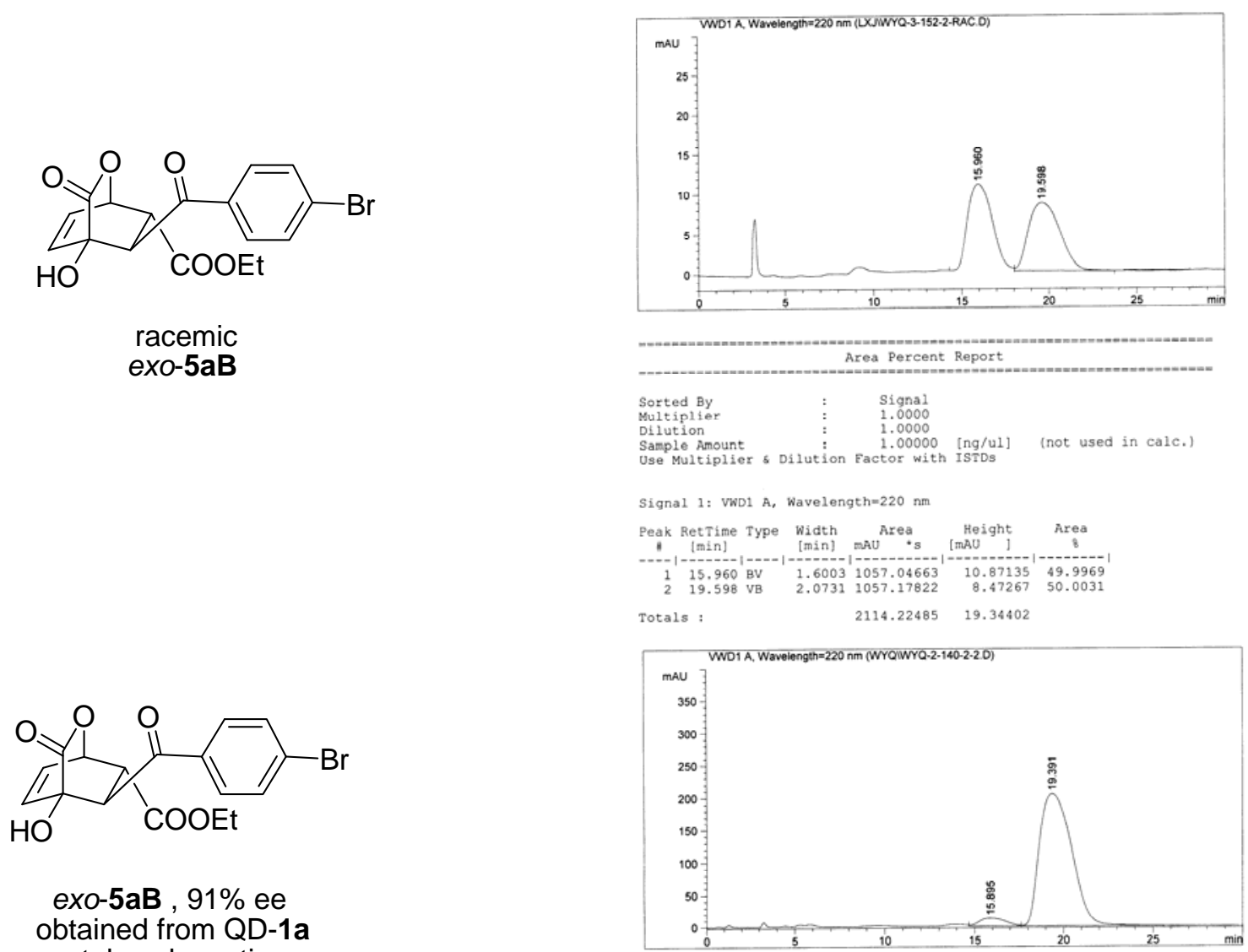

exo-5aB , 91\% ee obtained from QD-1a catalyzed reaction
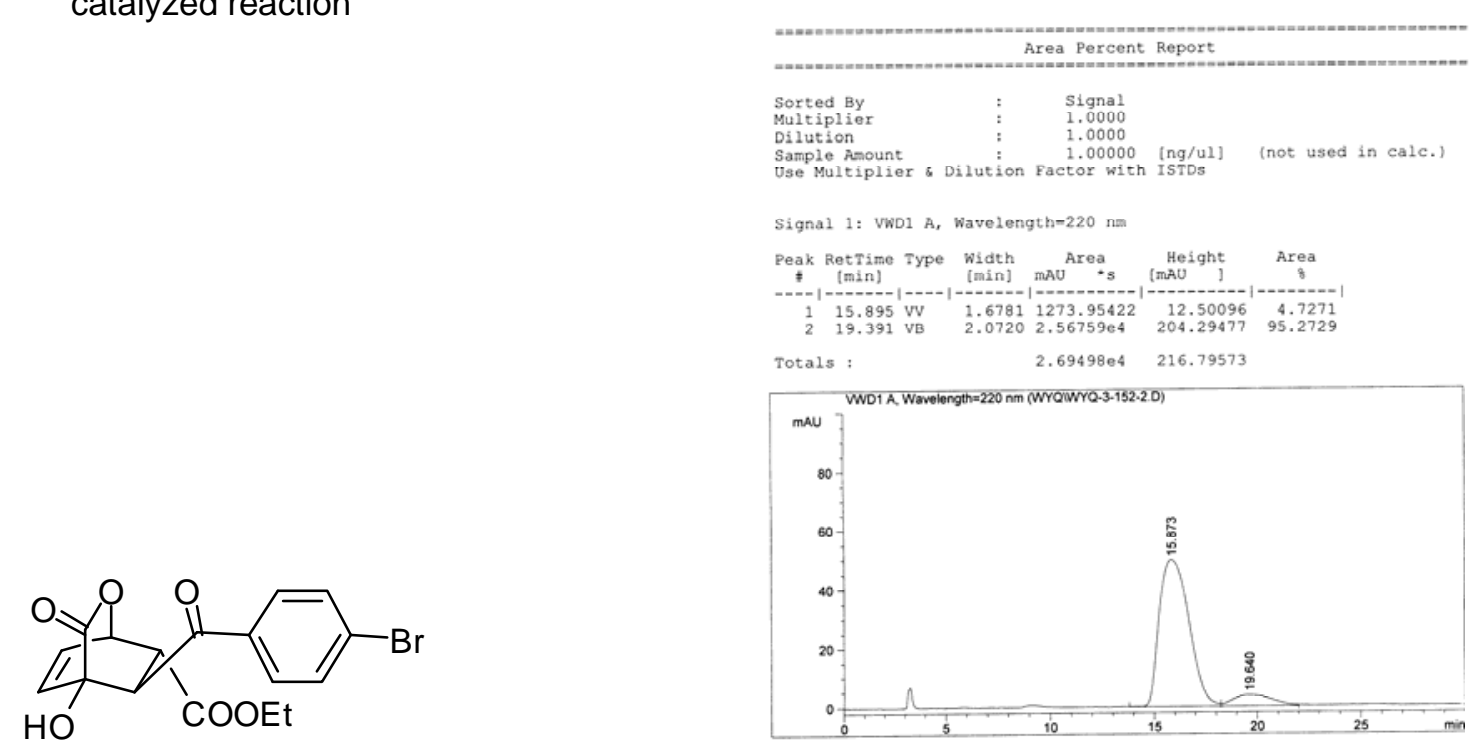

ent-exo-5aB , 83\% ee obtained from Q-1a catalyzed reaction

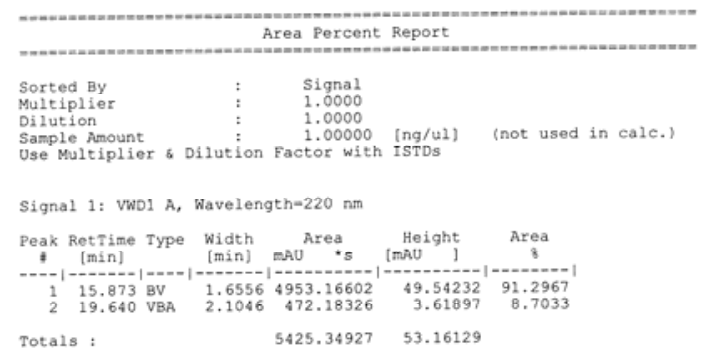


HPLC, Chiralpak AD, hexanes: isopropanol $=60: 40,1.0 \mathrm{~mL} / \mathrm{min}, \lambda=220 \mathrm{~nm}$

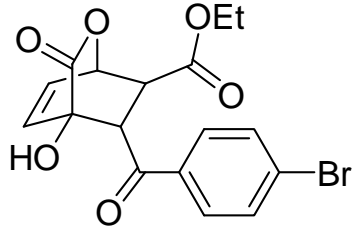

racemic endo-5aB

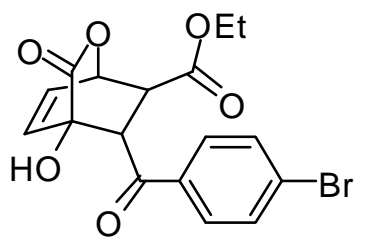

endo-5aB , $53 \%$ ee obtained from QD-1a catalyzed reaction

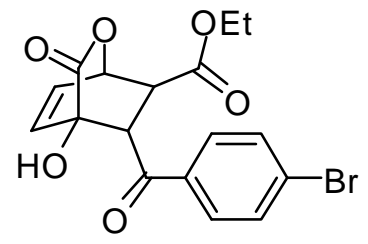

ent-endo-5aB , 25\% ee obtained from Q-1a catalyzed reaction
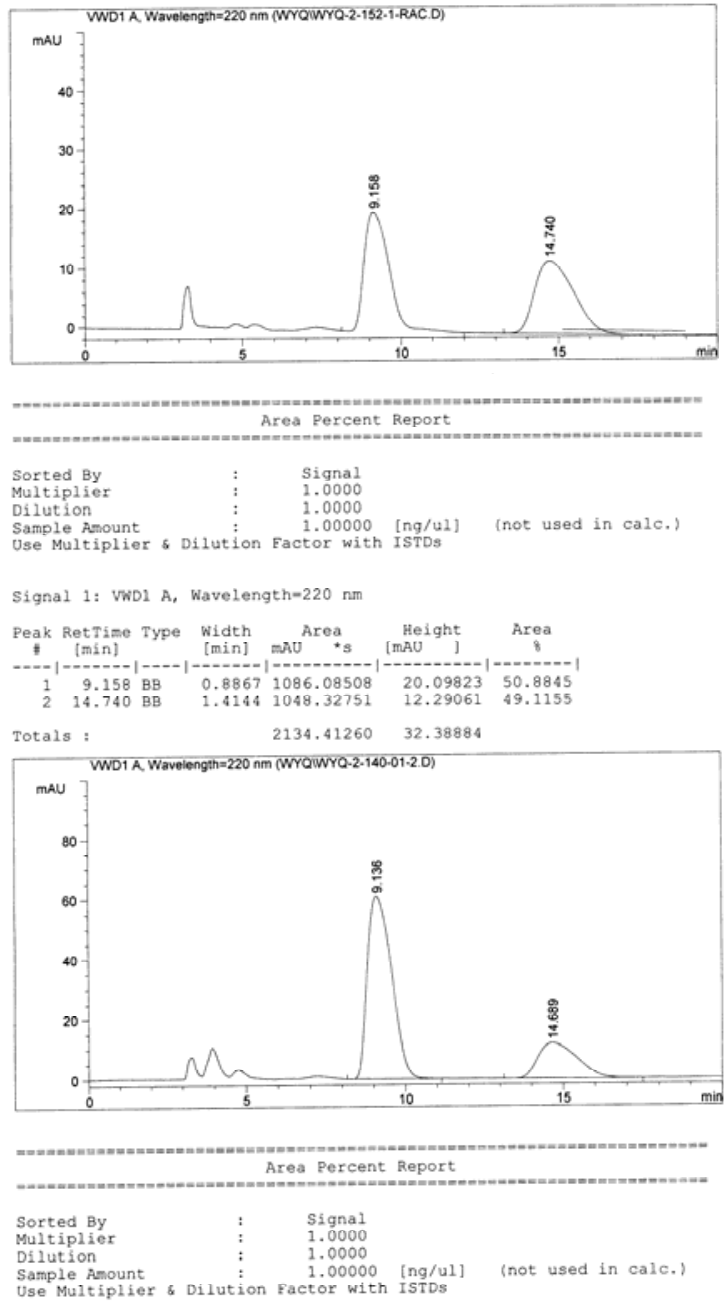

Signal 1: VWD1 $A$, wavelength $=220 \mathrm{~nm}$
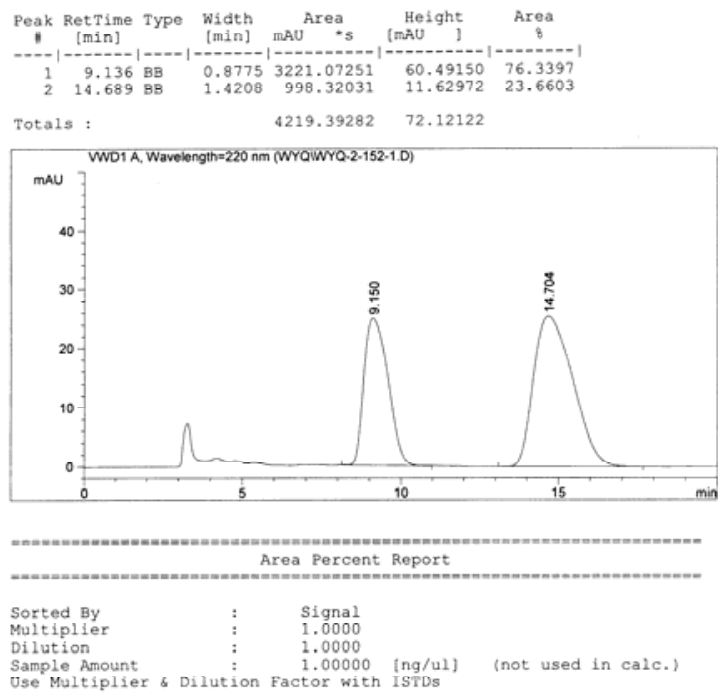

Sample Amount
Use Multiplier o Dilution Factor with ISTDs

Signal 1: VWD1 $\mathrm{A}$, wavelength-220 $\mathrm{nm}$

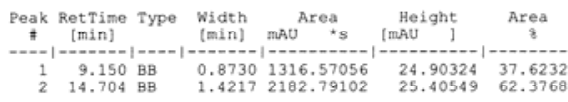

Totals : $\quad 3499.36157 \quad 50.30874$ 


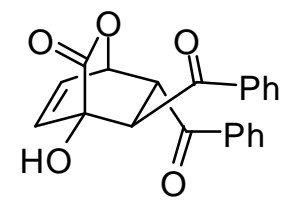

$5 \mathrm{aC}$

exo:endo $=93: 7$
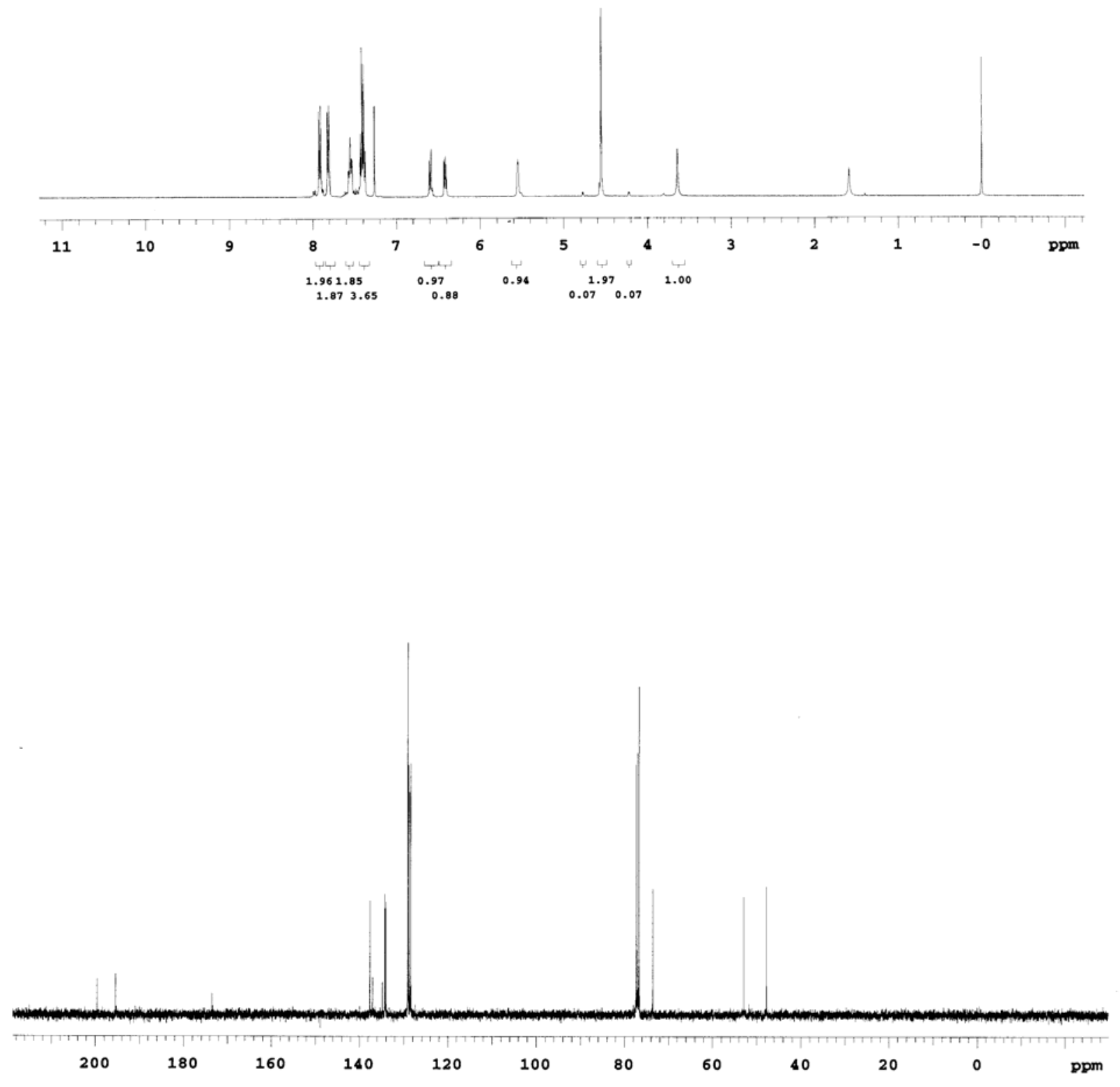
HPLC, Chiralpak AD, hexanes: isopropanol $=80: 20,1.0 \mathrm{~mL} / \mathrm{min}, \lambda=220 \mathrm{~nm}$

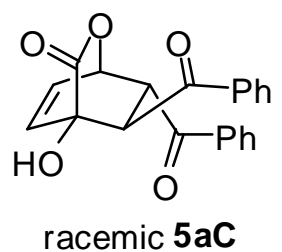

racemic $5 \mathrm{aC}$

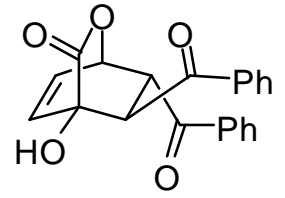

5aC, $\mathrm{dr}=93: 7,90 \%$ ee for exo adduct obtained from QD-1a catalyzed reaction
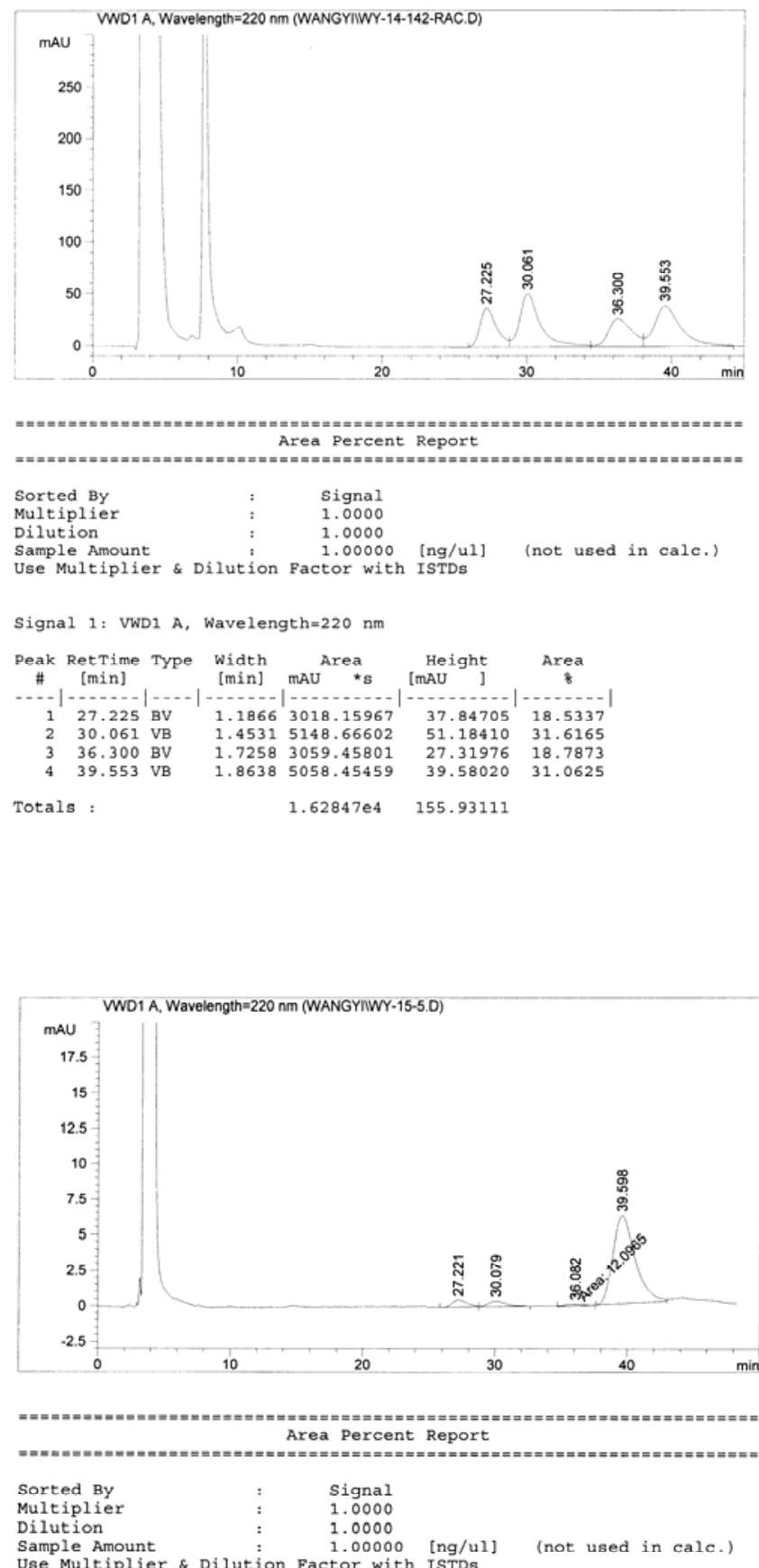

Use Multiplier \& Dilution Factor with ISTD

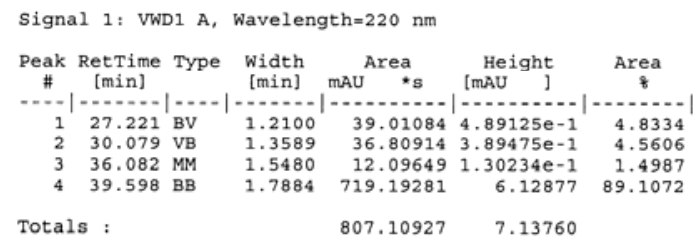



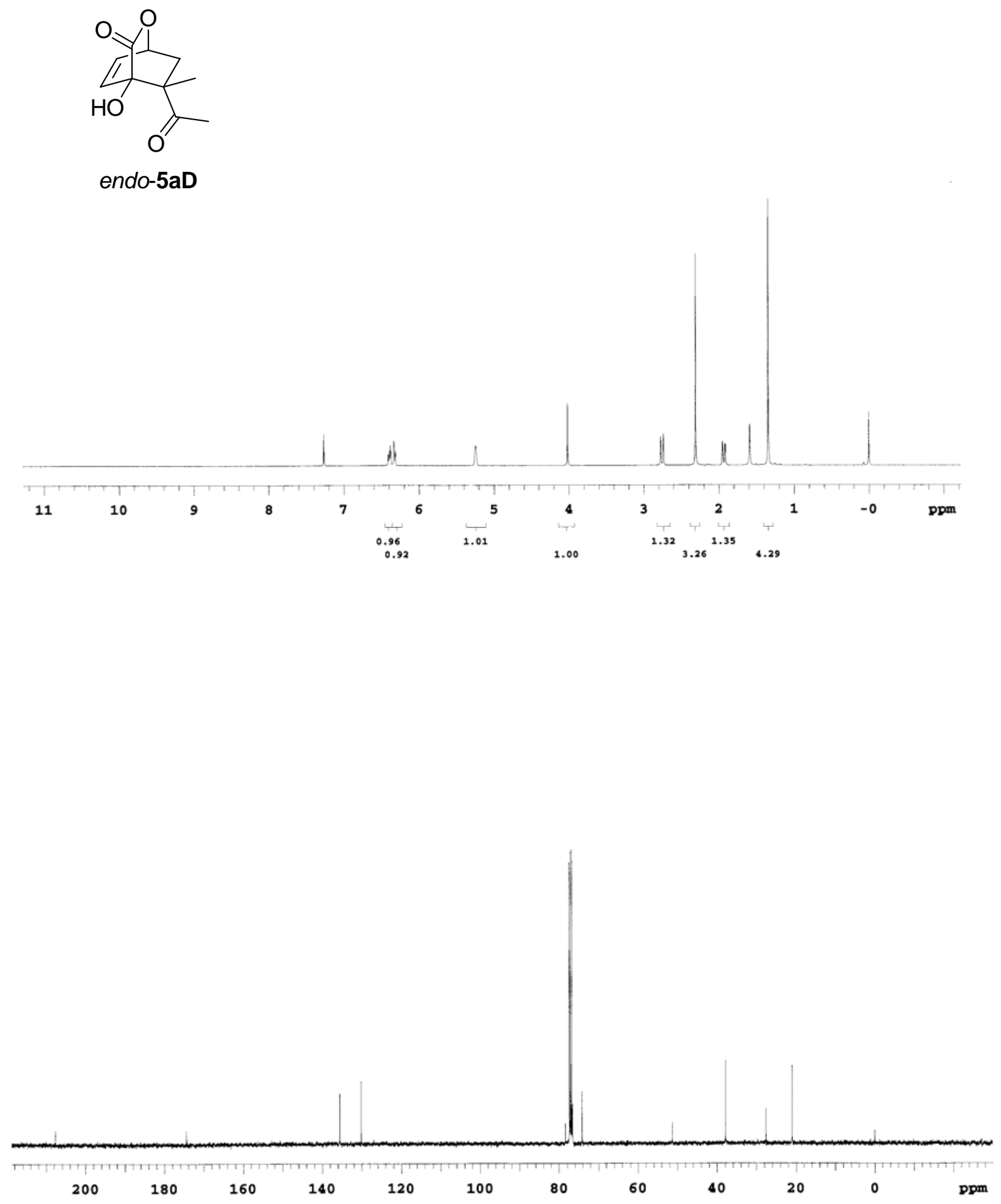

S 33 

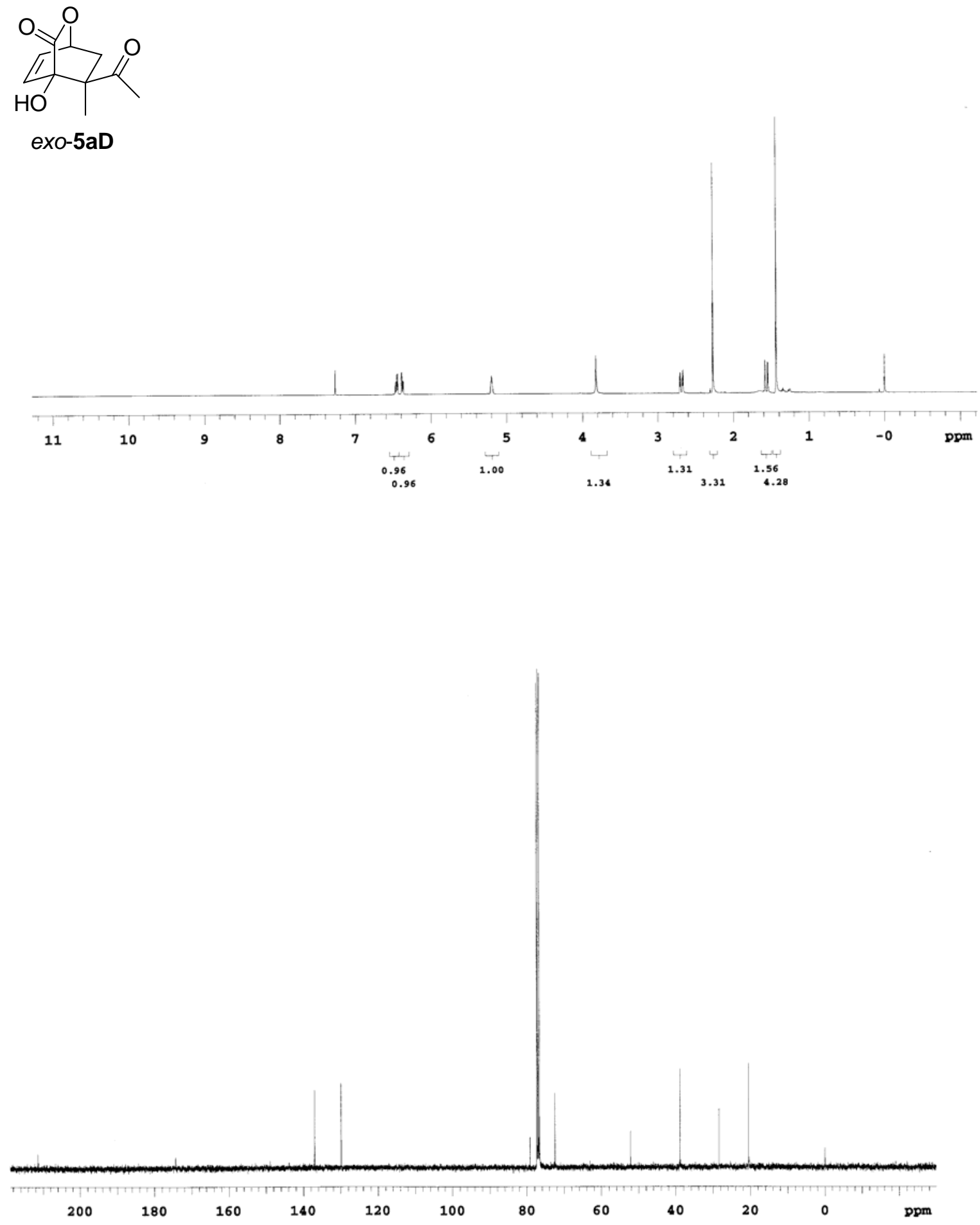
HPLC, Chiralcel OD, hexanes: isopropanol $=95: 5,1.0 \mathrm{~mL} / \mathrm{min}, \lambda=220 \mathrm{~nm}$

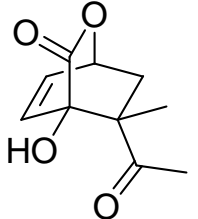

racemic

endo-5aD

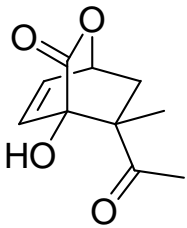

endo-5aD, 91\% ee obtained from QD-1a catalyzed reaction

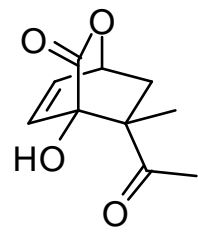

ent-endo-5aD, 90\% ee obtained from Q-1a catalyzed reaction
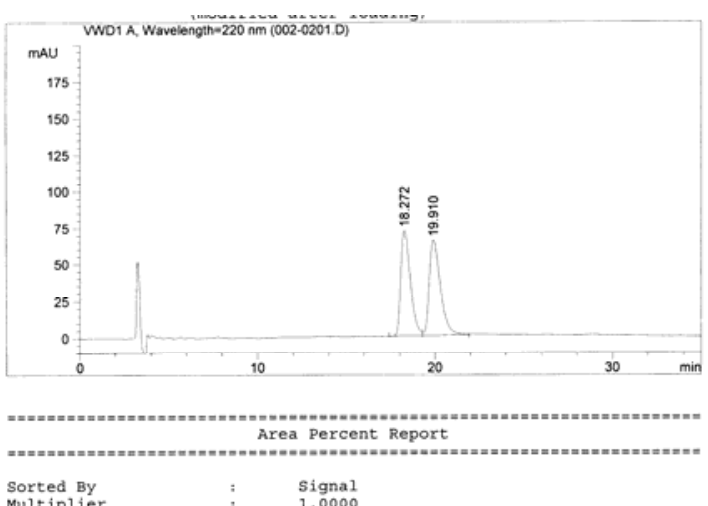

Dilution $\quad 1.0000$

Use Multiplier \& Dilution Factor with ISTD

Signal 1: VWD1 $A$, wavelength $=220 \mathrm{~nm}$

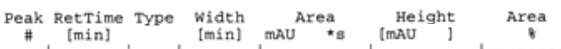

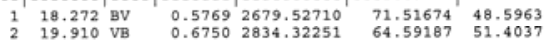

$\begin{array}{lll}\text { Totals : } & 5513.84961 \quad 136.10861\end{array}$
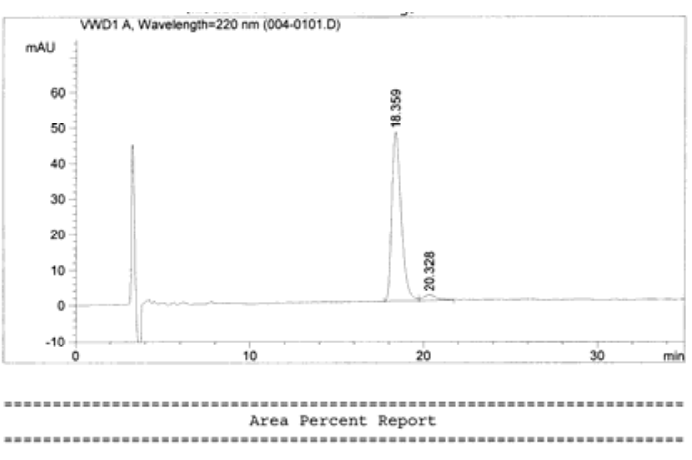

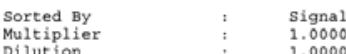

$\begin{array}{lll}\text { Sorted By } & \text { Signal } \\ \text { Multiplier } & \vdots & 1.0000 \\ \text { Dilution } & 1 & 1.0000\end{array}$

Dilution
Use Multiplier \& Dilution Factor with ISTDE

Signal 1: VwD1 $A$, wavelength=220 nm

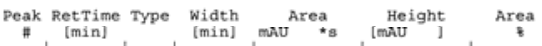

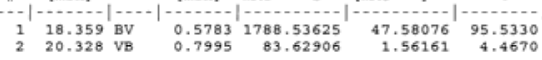

Totals : $\quad 1872.16531 \quad 49.14238$
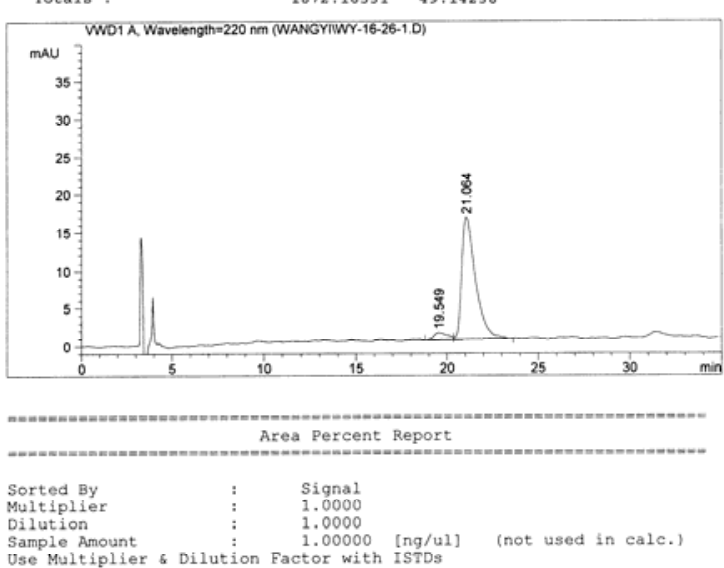

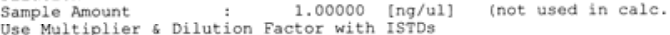

Signal 1: VWD1 $A$, wavelength-220 nm

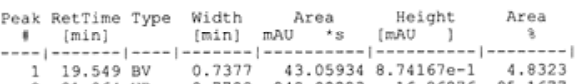

$\begin{array}{lll}\text { Totals : } & 891.08216 & 16.94293\end{array}$ 
HPLC, Chiralpak AS hexane:isopropanol $=70: 30,1.0 \mathrm{~mL} / \mathrm{min}, \lambda=220 \mathrm{~nm}$

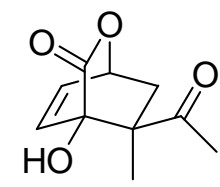

racemic

exo-5aD

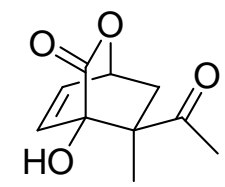

exo-5aD, 95\% ee obtained from QD-1a catalyzed reaction

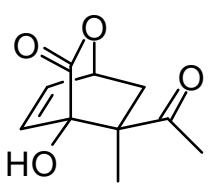

ent-exo-5aD, 93\% ee obtained from Q-1a catalyzed reaction
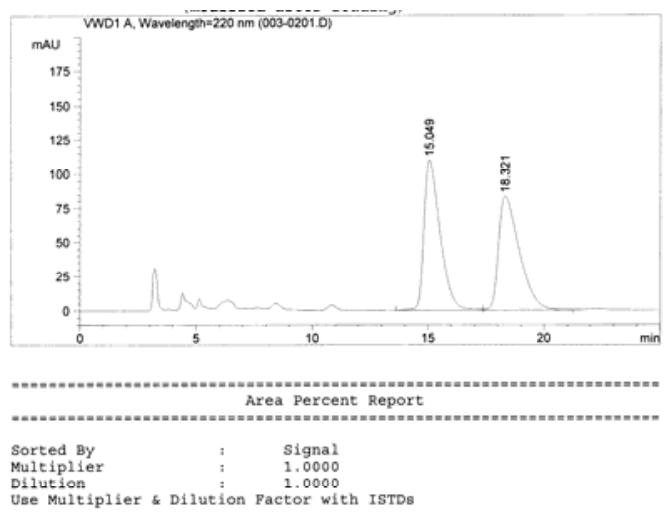

Signal 1: VWD1 $A$, wavelength $=220 \mathrm{~nm}$

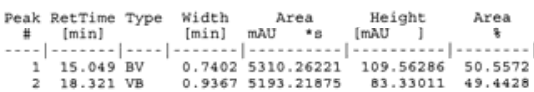

Totals : $\quad 1.05035 \mathrm{e} \quad 192.89297$
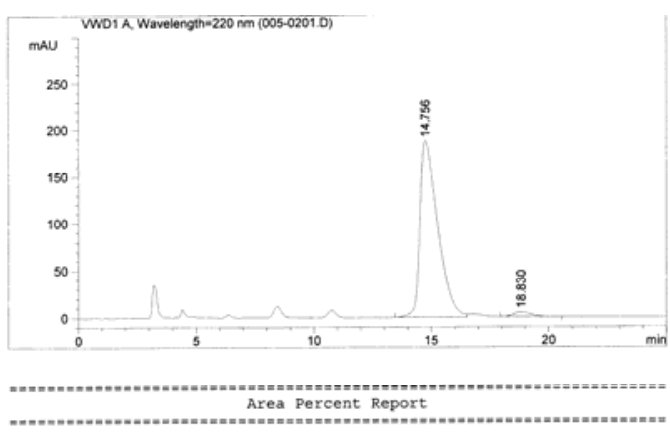

Sorted By
Multiplier

Signal
1.0000

Dilution
Use Multiplier \& Dilution Factor with ISTD

Signal 1: VWD1 $A$, wavelength $220 \mathrm{~nm}$

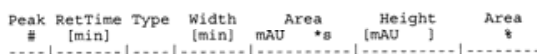

$\begin{array}{rrrrrr}14.756 \mathrm{BBA} & 0.7876 & 9850.73730 & 187.50308 & 97.4360 \\ 2 & 18.830 \mathrm{BB} & 0.7971 & 259.21881 & 5.04823 & 2.5640\end{array}$

Totals: $\quad 1.01100 \mathrm{e} \quad 192.55131$

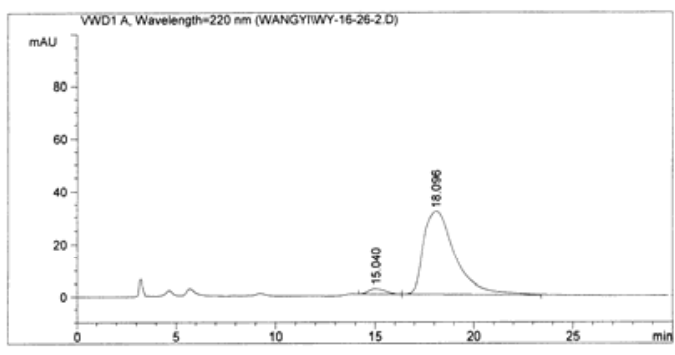

Area Percent Repor

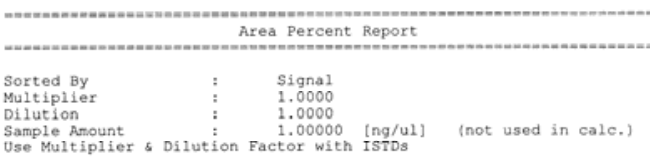

Sample Amount
Use Multiplier s Dilution Factor with ISTDs
1.00000
[ng/ul] (not used in calc.

Signal 1: vwD1 $A$, wavelength $=220 \mathrm{~nm}$

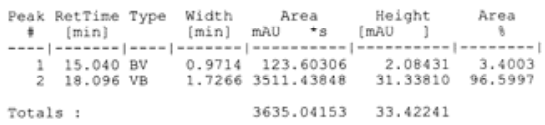



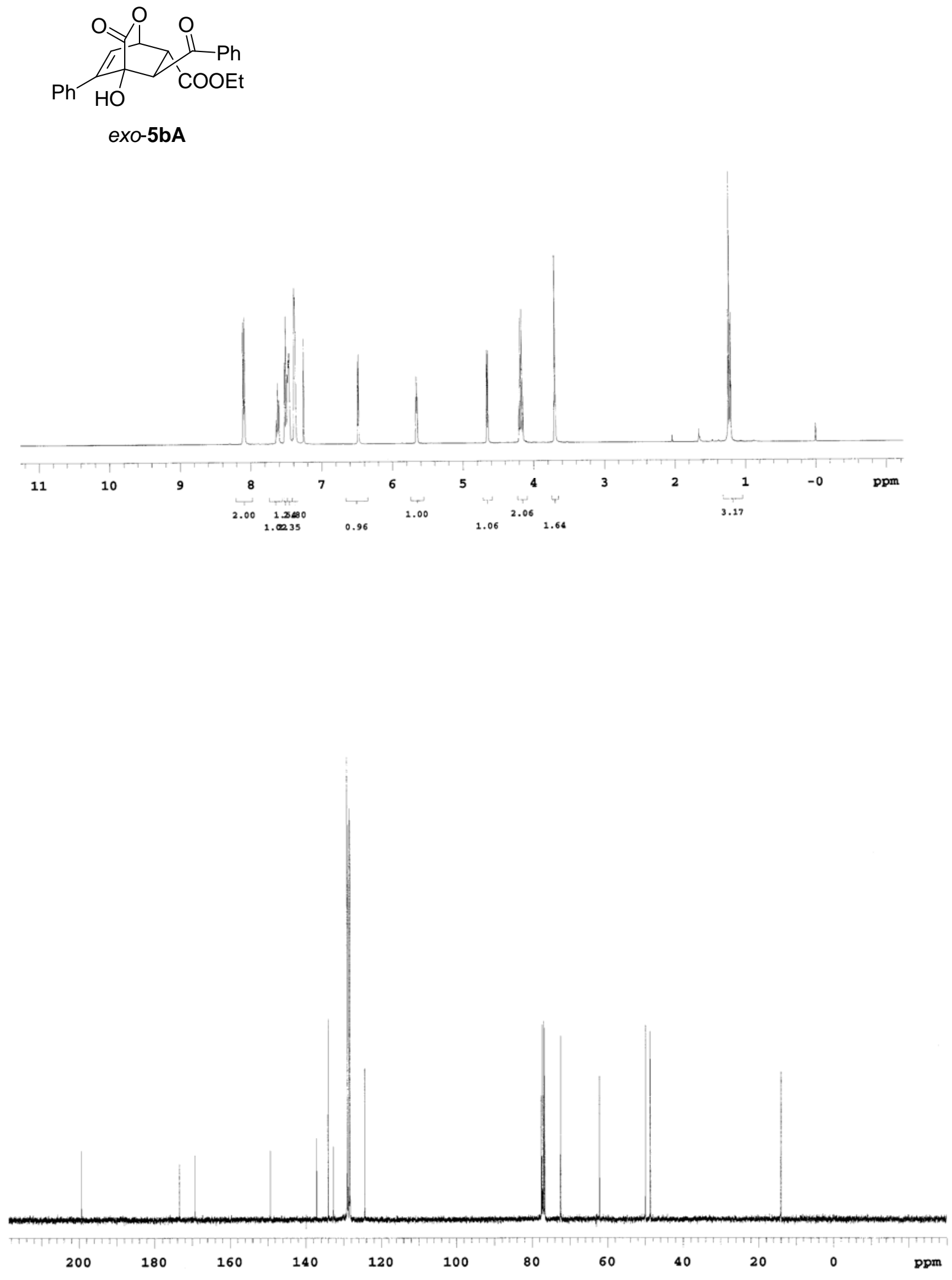
HPLC, $(R, R)$-Whelk-O1, hexanes: isopropanol $=90: 10,0.8 \mathrm{~mL} / \mathrm{min}, \lambda=220 \mathrm{~nm}$

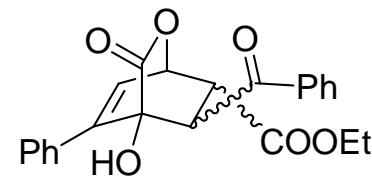

Racemic 5bA

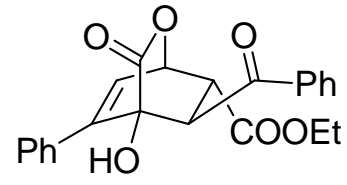

exo-5bA , 85\% ee obtained from QD-1a catalyzed reaction
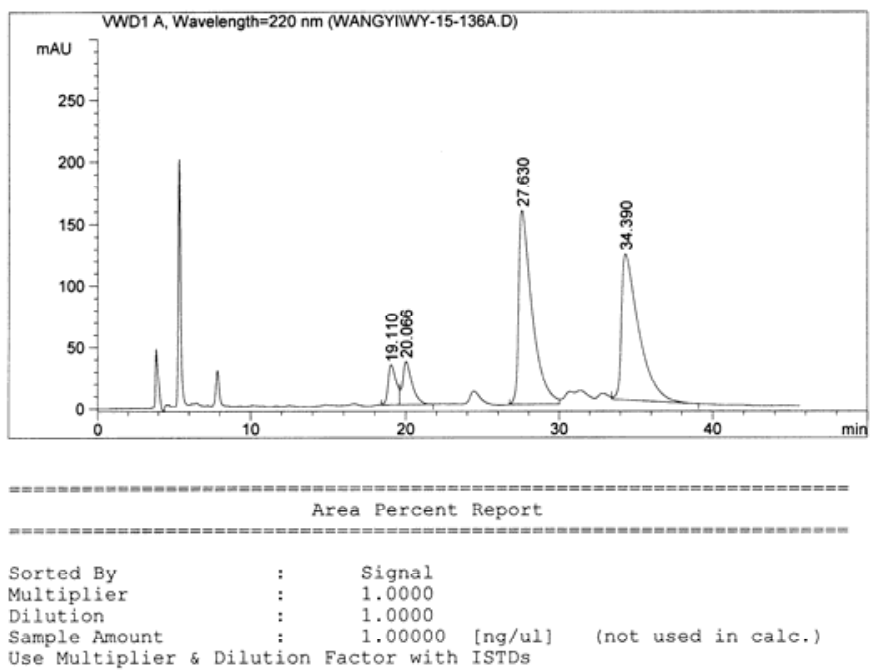

Use Multiplier \& Dilution Factor with ISTDs

Signal 1: VWD1 $A$, Wavelength $=220 \mathrm{~nm}$
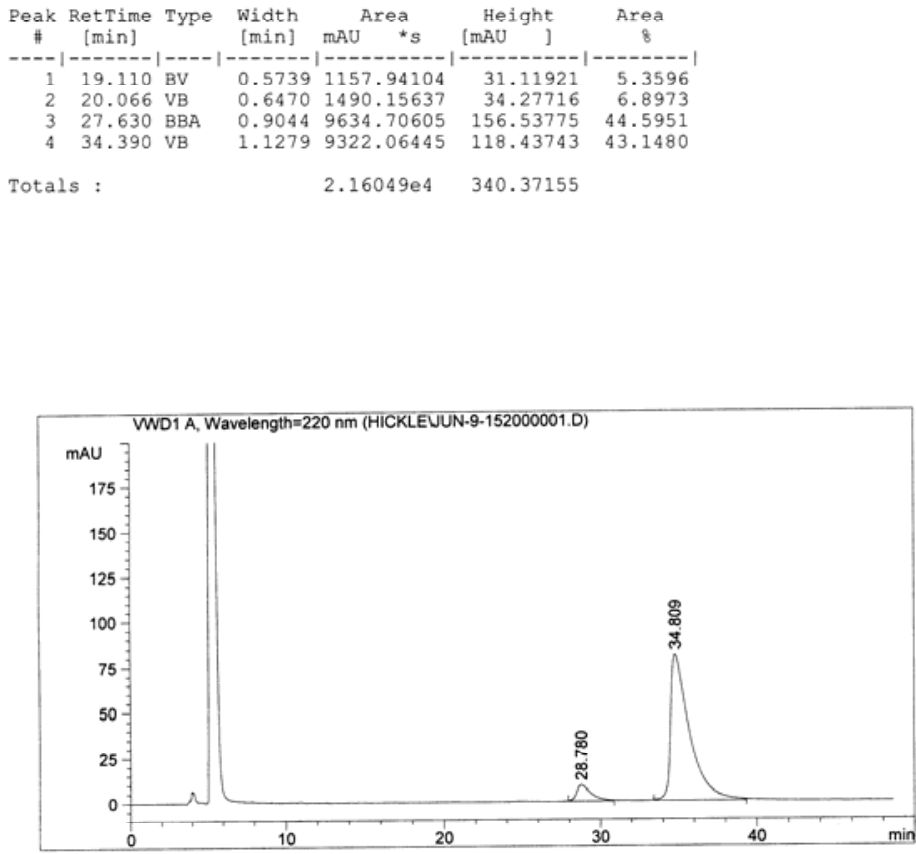

Area Percent Report

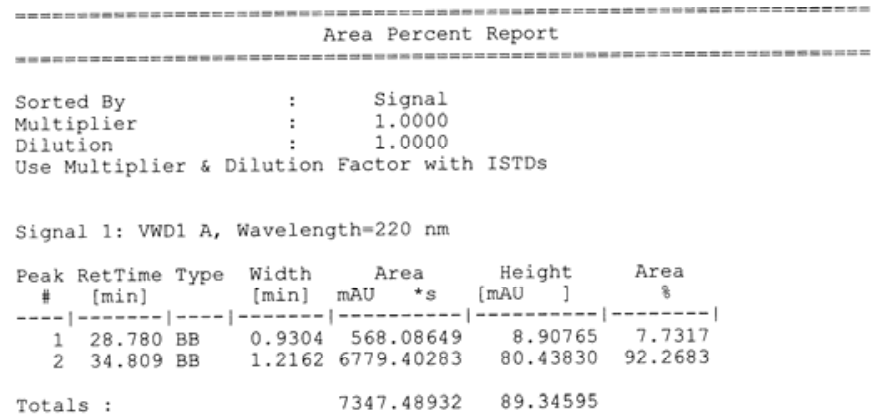



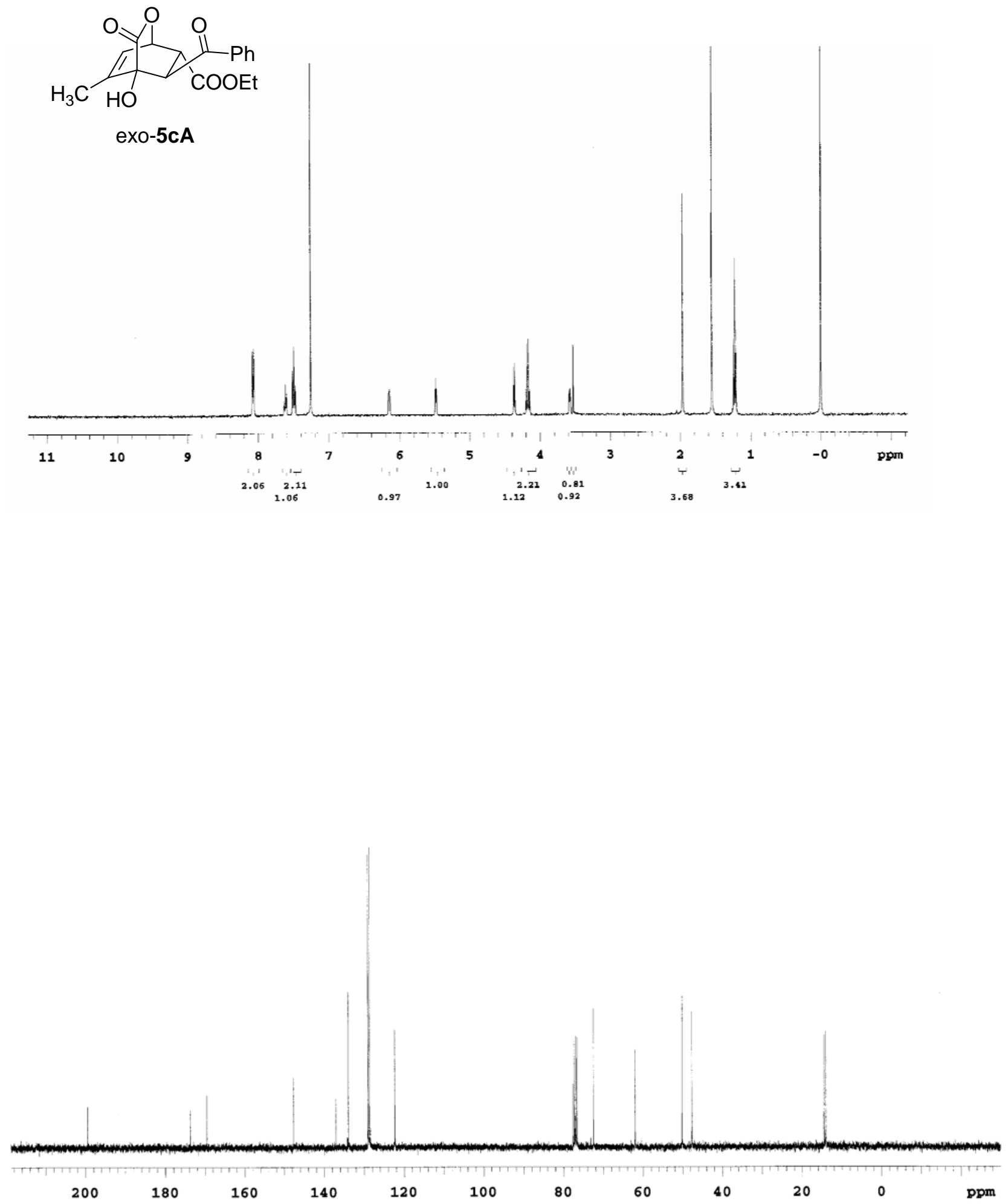

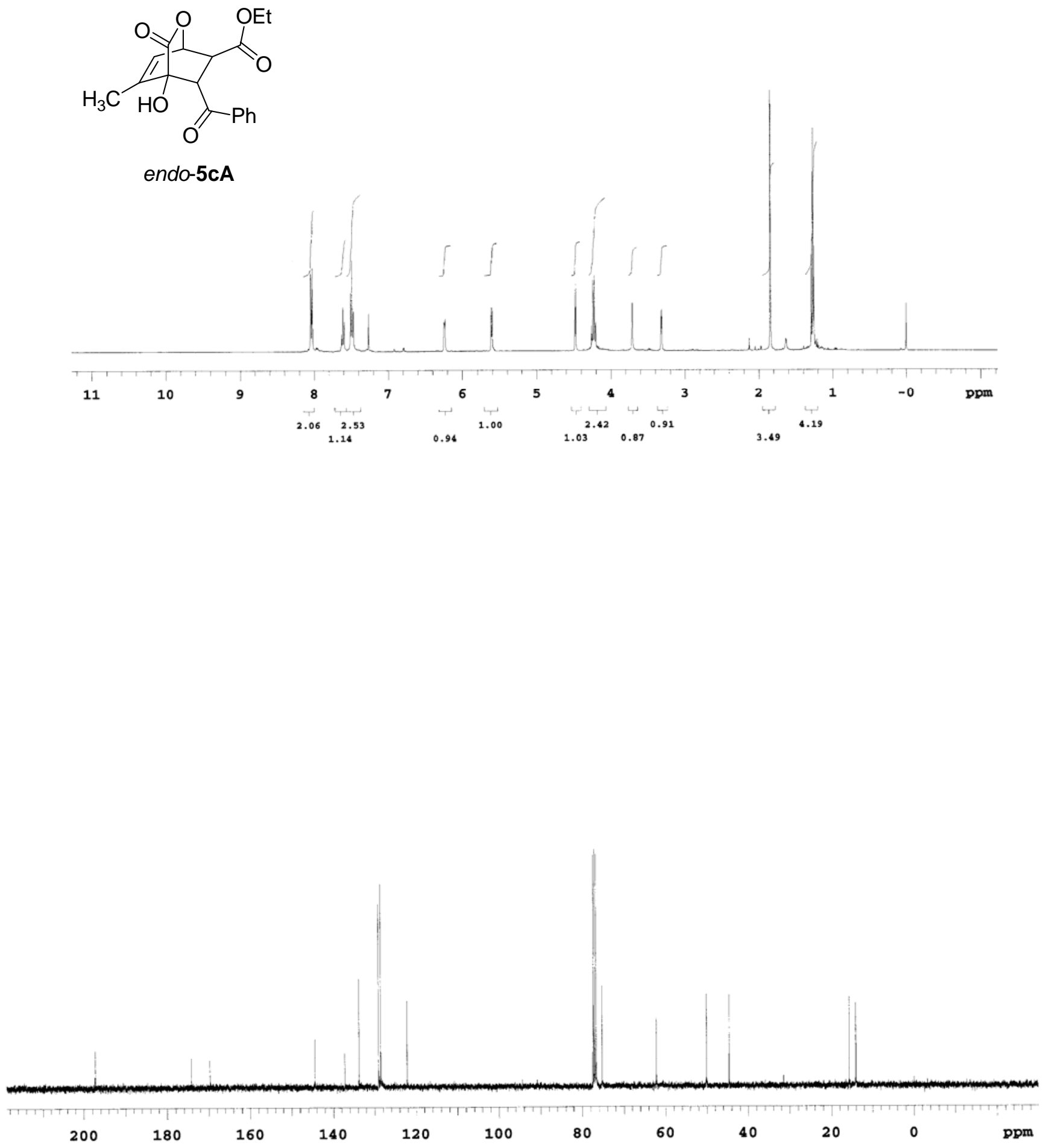
HPLC, Chiralcel OD, hexanes: isopropanol $=85: 15,0.8 \mathrm{~mL} / \mathrm{min}, \lambda=220 \mathrm{~nm}$

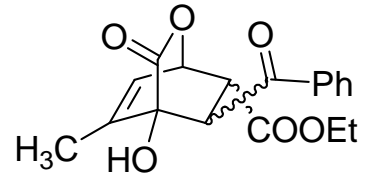

racemic-5aC

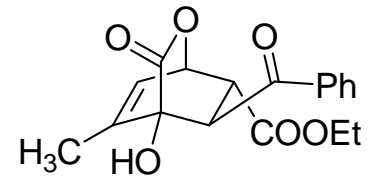

exo-5aC, $82 \%$ ee obtained from QD-1a catalyzed reaction<smiles>CCOC(=O)C1C2OC(=O)C(C)(C(C)=O)C1O2</smiles>

endo-5ac, $36 \%$ ee obtained from QD-1a catalyzed reaction
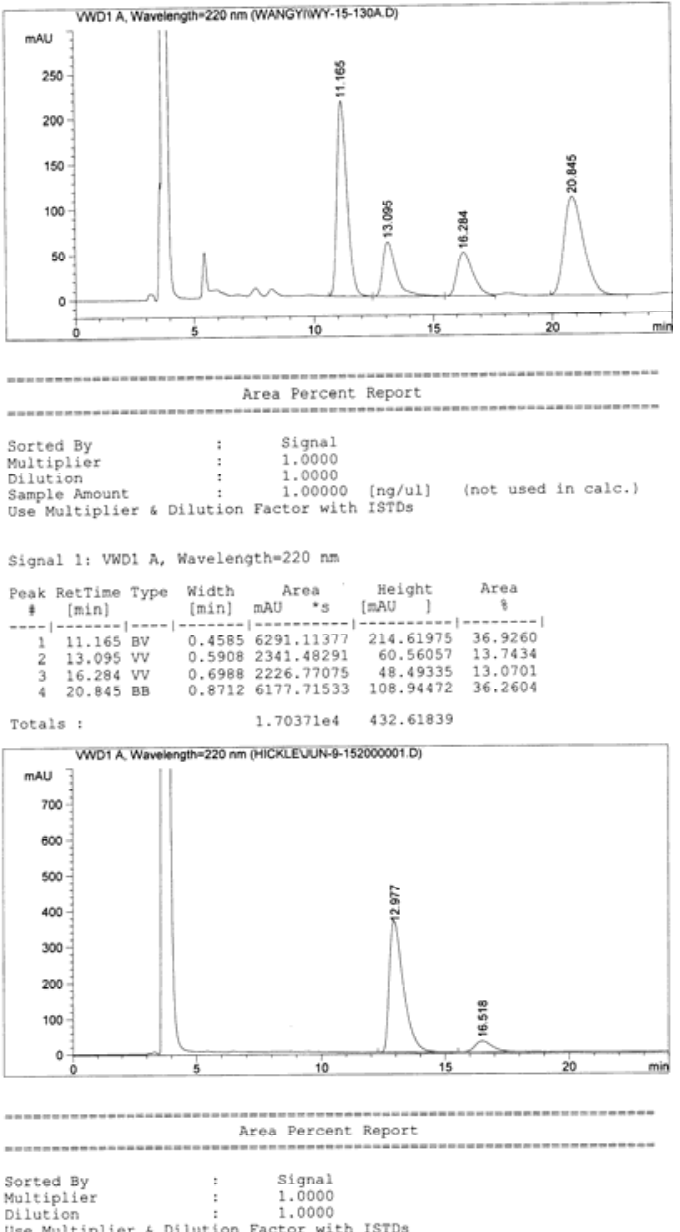

Use Multiplier s Dilution Factor with ISTDe

Eignal 1: WWD1 $\mathrm{A}$, Wavelengthm $220 \mathrm{~nm}$
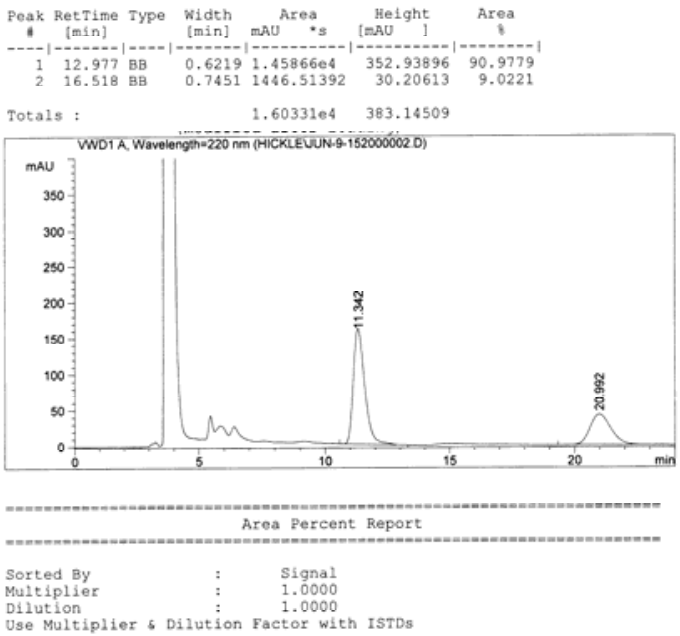

Use Multiplier s Dilution Factor with ISTDs

Signal 1: VWD1 $\mathrm{A}$, wavelength-220 $\mathrm{nm}$

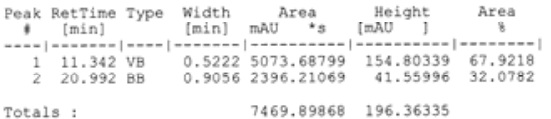



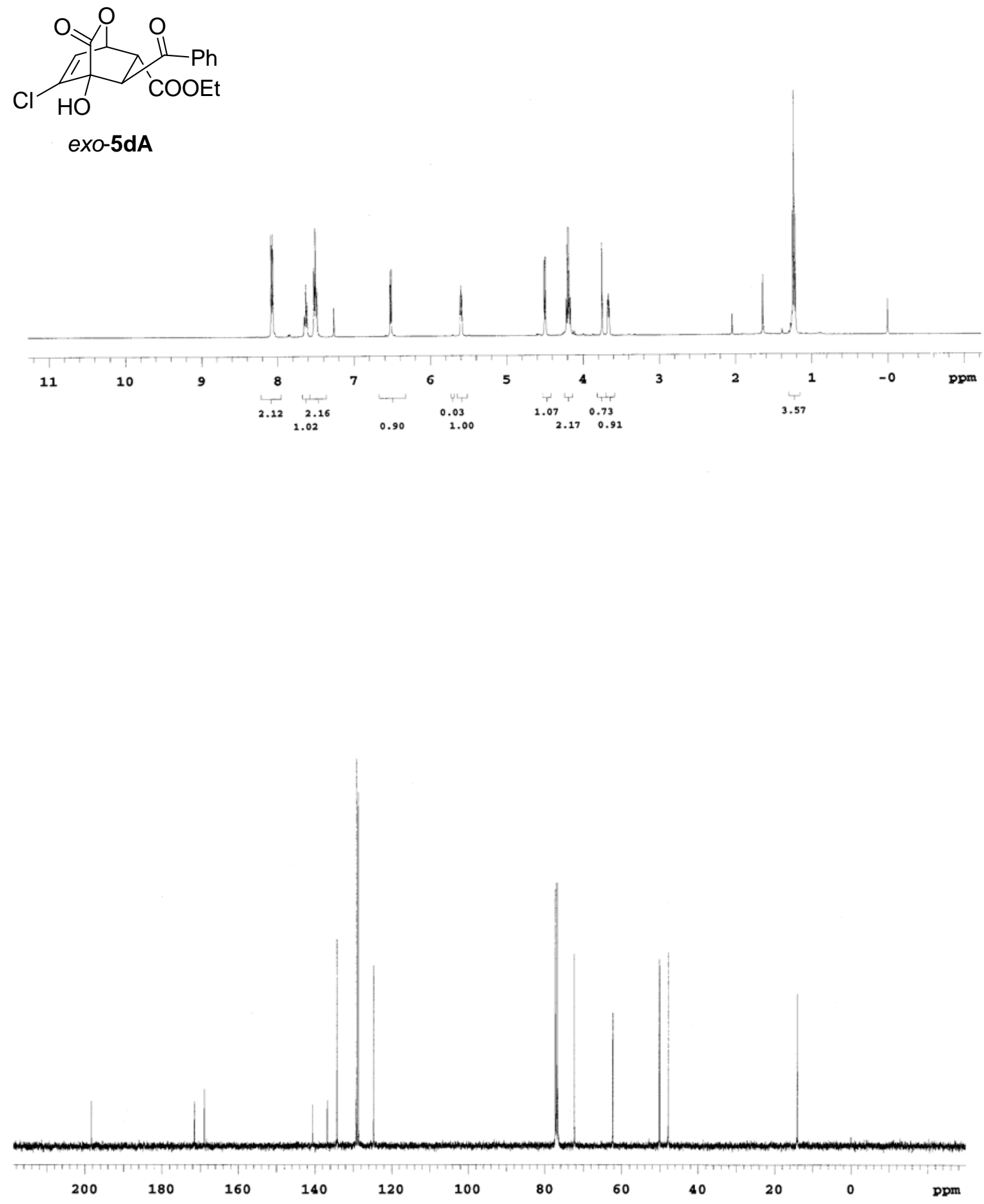


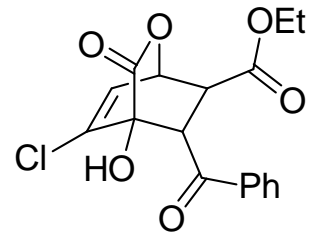

endo-5dA
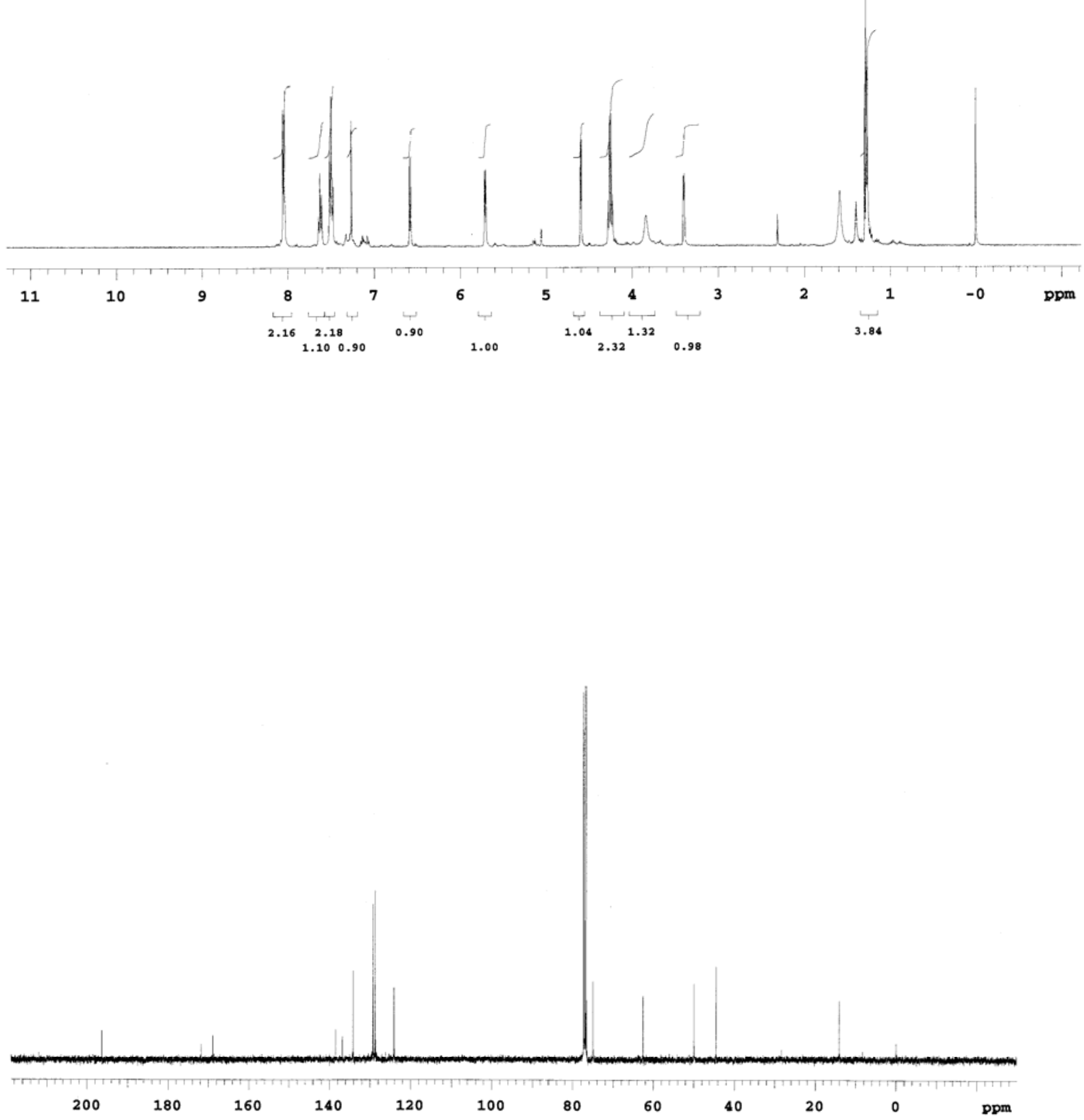
HPLC, Chiralcel OJ, hexanes: isopropanol $=70: 30,0.8 \mathrm{~mL} / \mathrm{min}, \lambda=254 \mathrm{~nm}$.

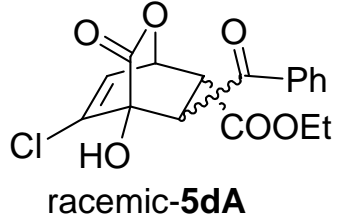

${ }_{\mathrm{HO}}^{\mathrm{O}} \mathrm{Cl}_{\mathrm{COOEt}}^{\mathrm{O}}$ exo-5dA, 84\% ee obtained from QD-1a catalyzed reaction

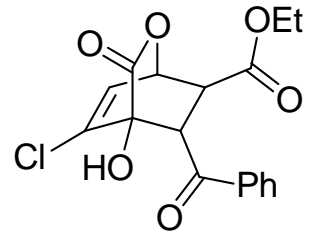

endo-5dA, 1\% ee obtained from QD-1a catalyzed reaction
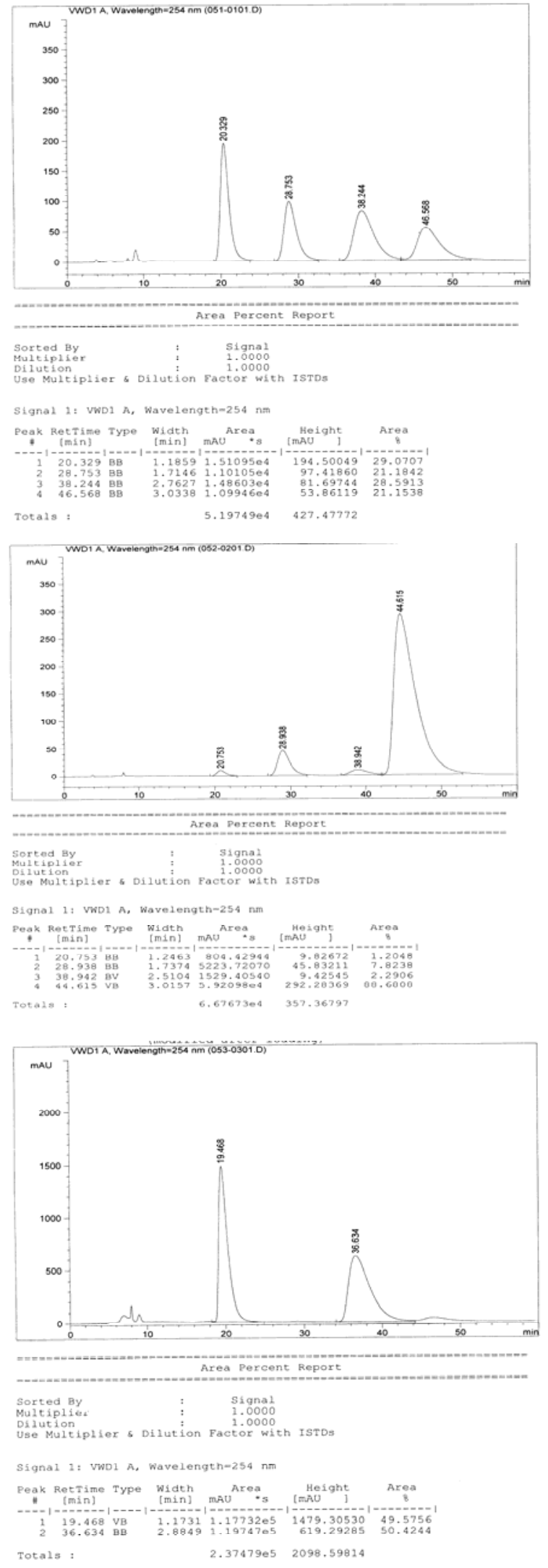

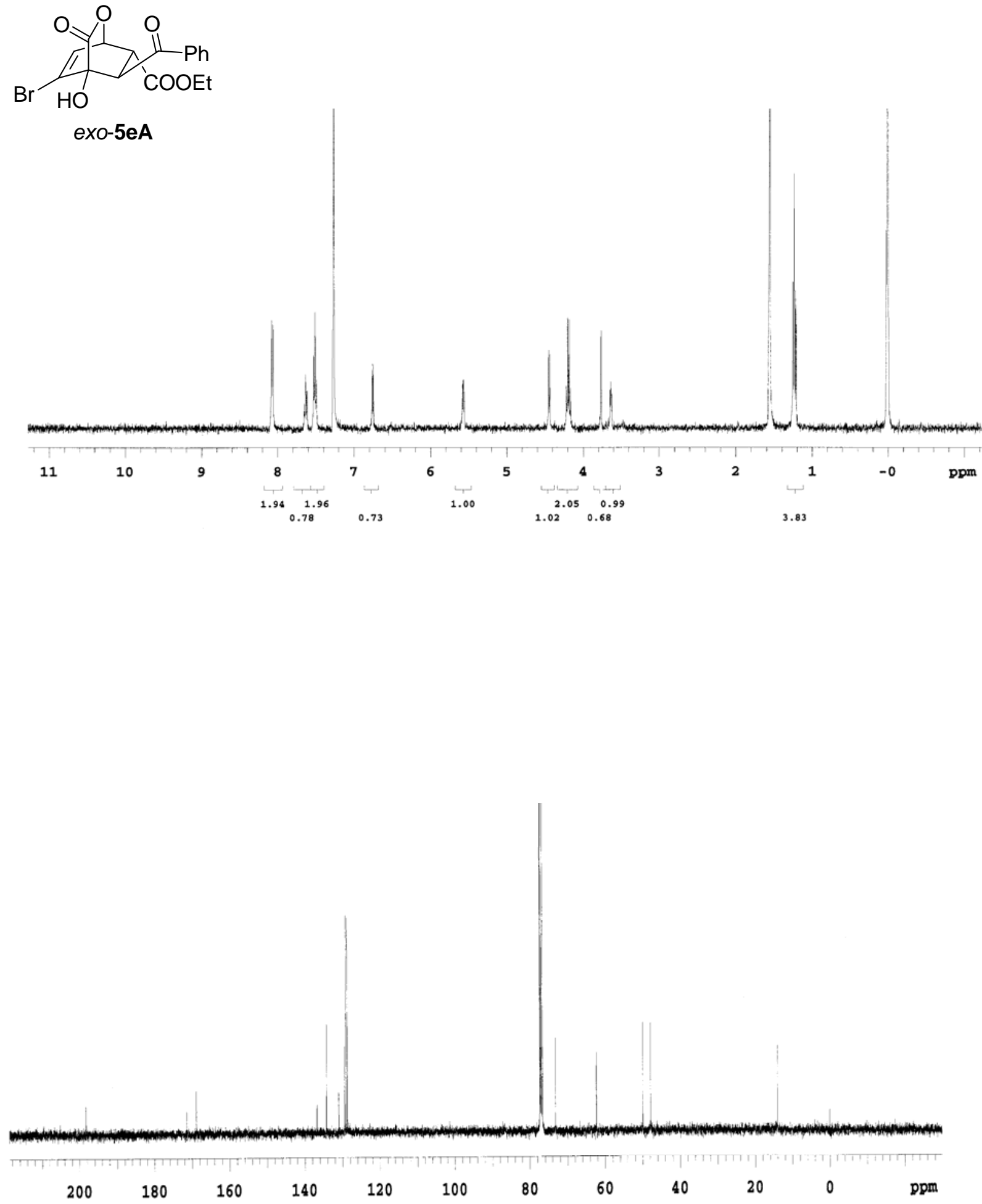

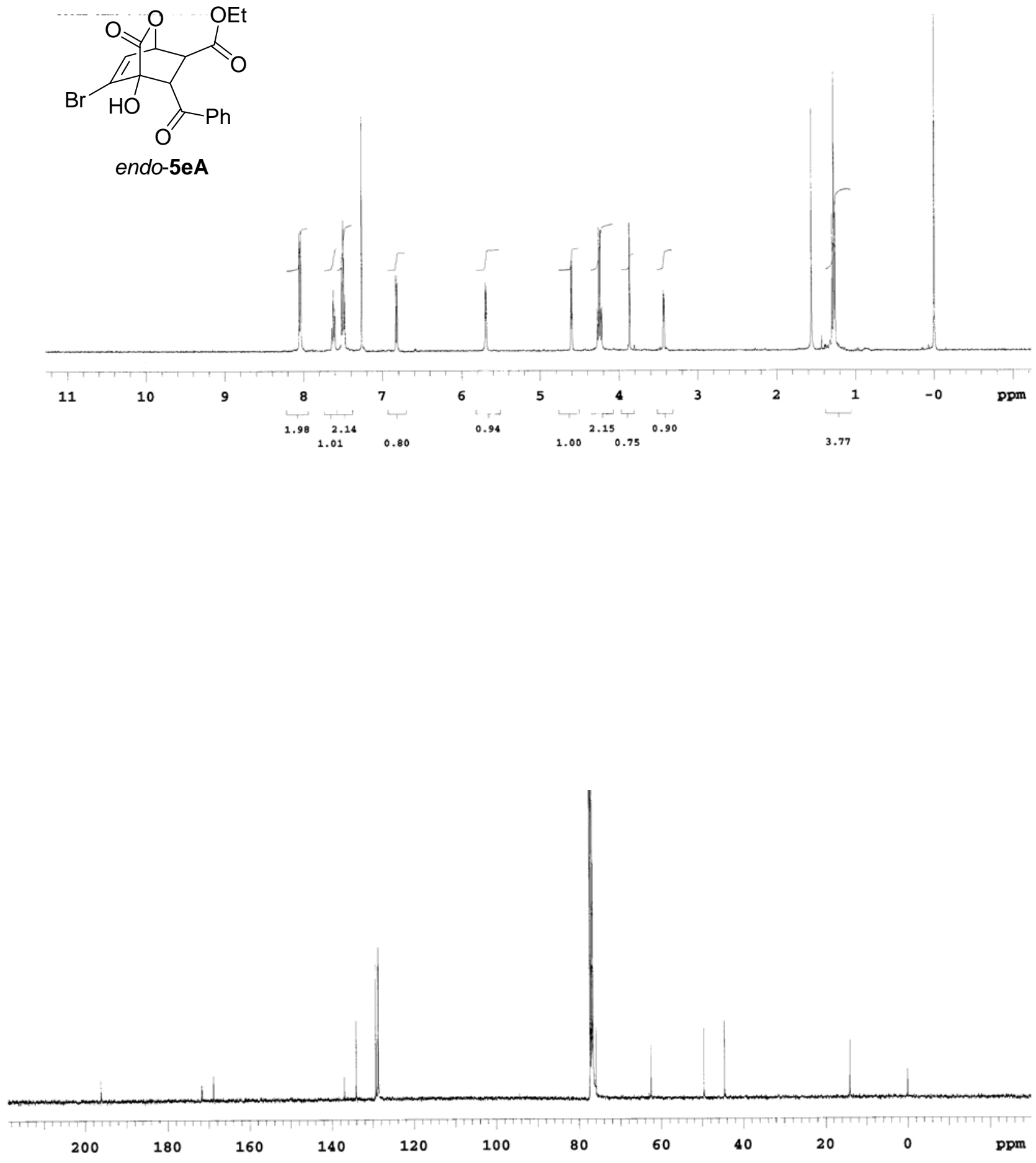
HPLC, Chiralcel OJ, hexanes: isopropanol $=70: 30,0.8 \mathrm{~mL} / \mathrm{min}, \lambda=220 \mathrm{~nm}$.

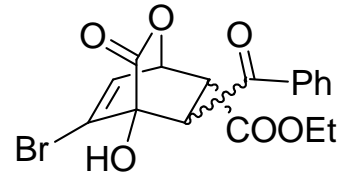

racemic-5eA<smiles>CCOC(=O)C1(C(=O)OCC)C2OC(=O)C1(O)C(Br)=C2Br</smiles>

exo-5eA, 83\% ee obtained from QD-1a catalyzed reaction

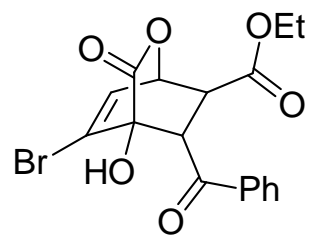

endo-5eA, 7\% ee obtained from QD-1a catalyzed reaction
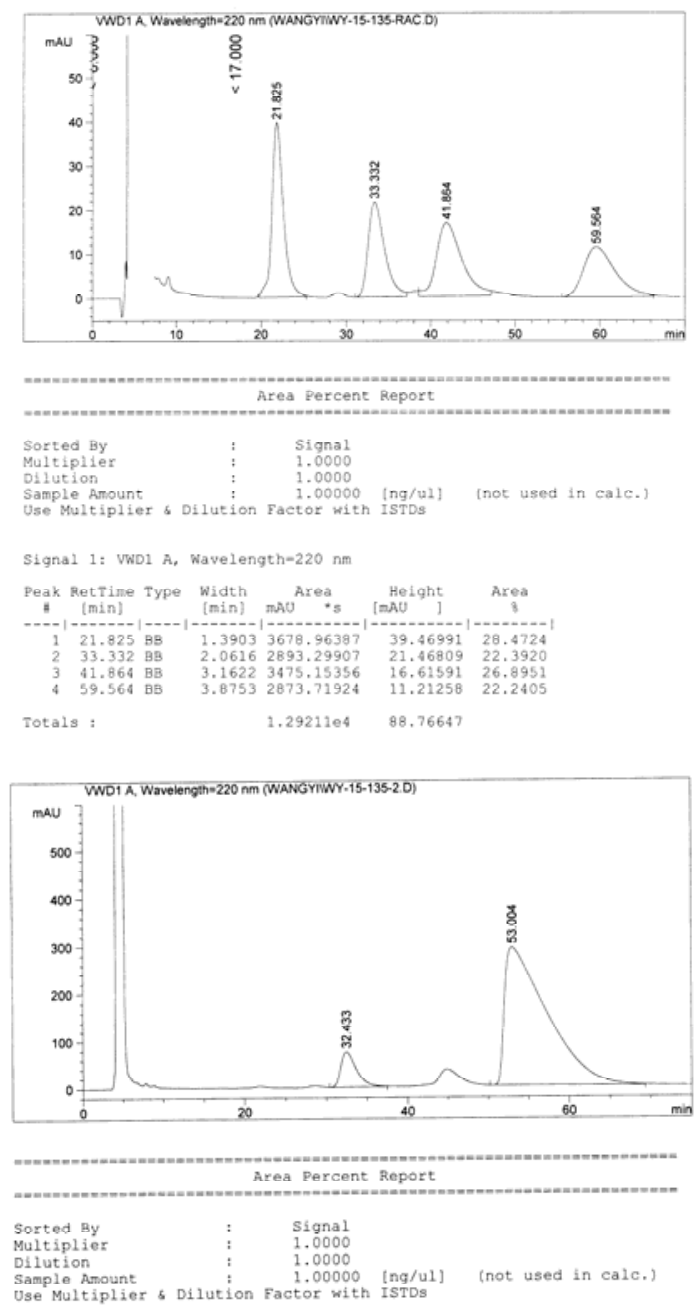

Sample Amount
Use Multiplier s Dilution Factor with ISTDs

Signal 1: VWD1 $A$, wavelength-220 nm

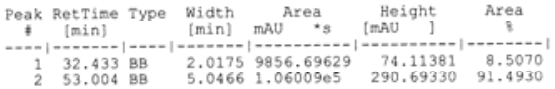

Totals : $\quad 1.15866 \mathrm{es} \quad 364.80711$
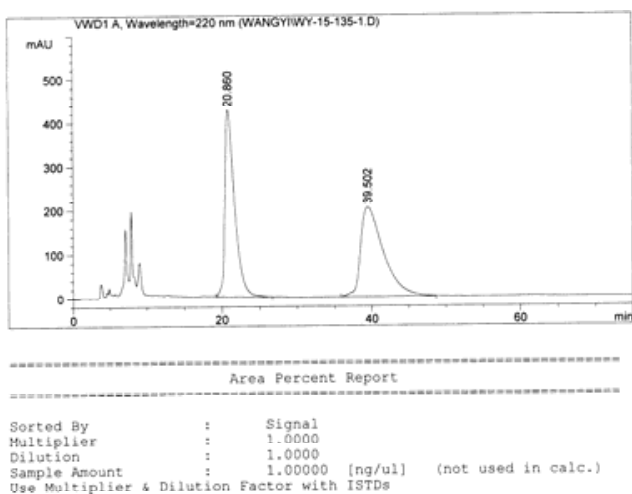

Signal 1: WWD1 A, Kavelength=220 nim

Peak RetTime Type width
$[$ Area
$[\min ]$

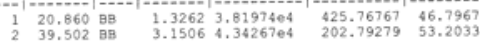

Totals : $\quad 8.16240 \mathrm{e} \quad 628.56046$ 

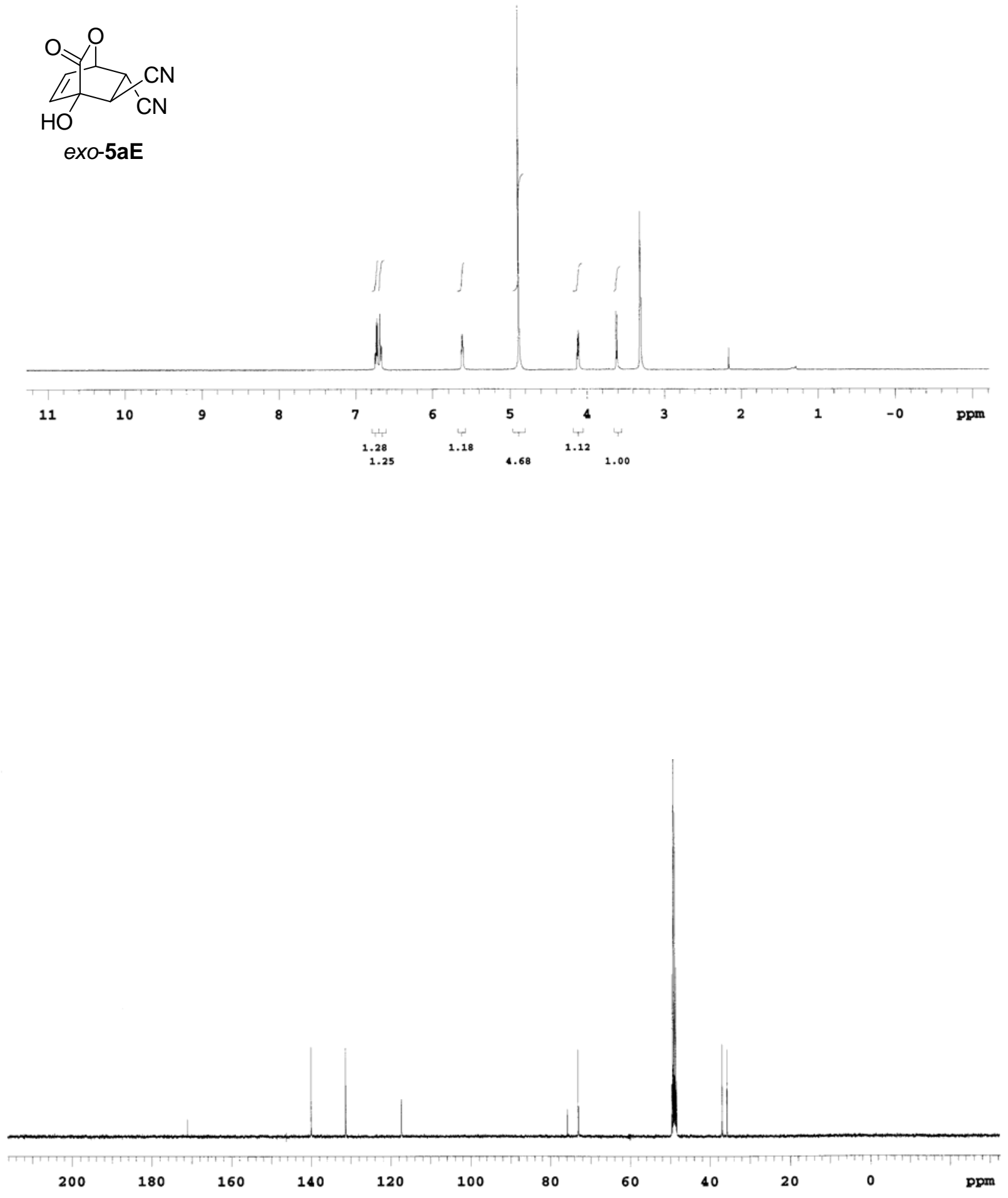
HPLC, Chiralpak AS, hexanes: isopropanol $=60: 40,0.6 \mathrm{~mL} / \mathrm{min}, \lambda=220 \mathrm{~nm}$

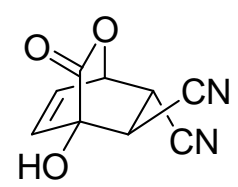

racemic 5aE

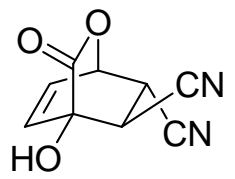

exo-5aE, 92\% ee obtained from QD-2 catalyzed reaction

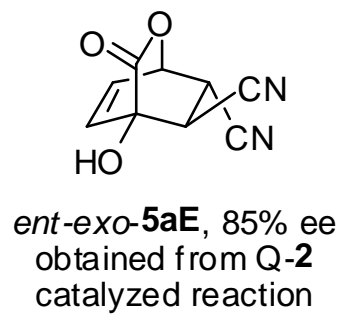

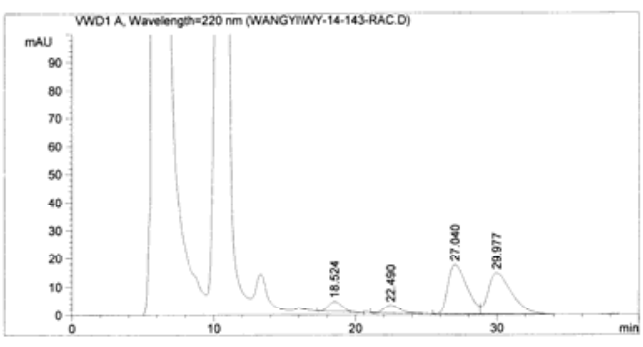

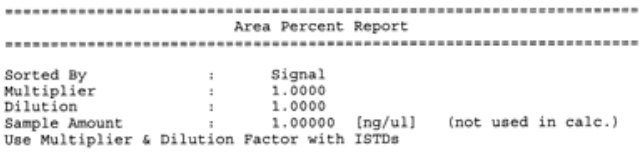

Signal 1: VWD1 $A$, wavelengthe $220 \mathrm{~nm}$

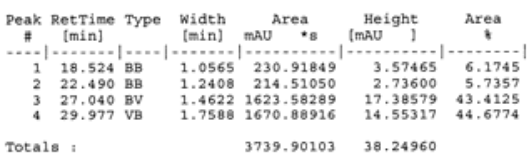

rotals: $3739.90103 \quad 38.24960$
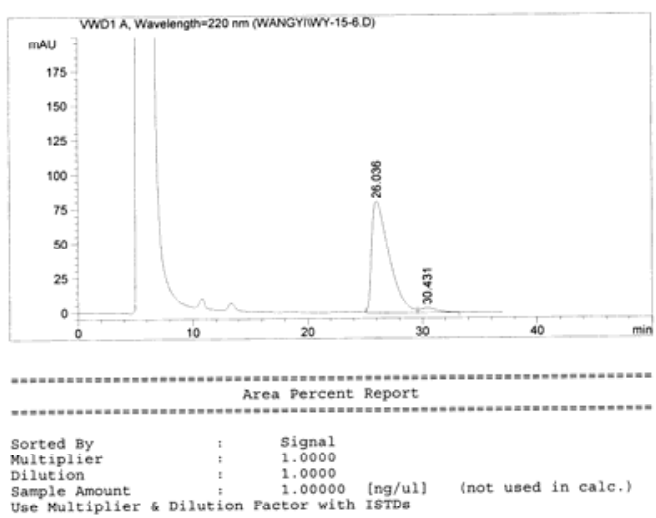

Signal 1: VWD1 A, wavelength=220 nm

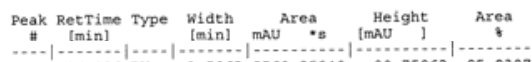

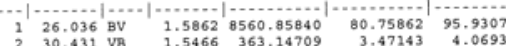
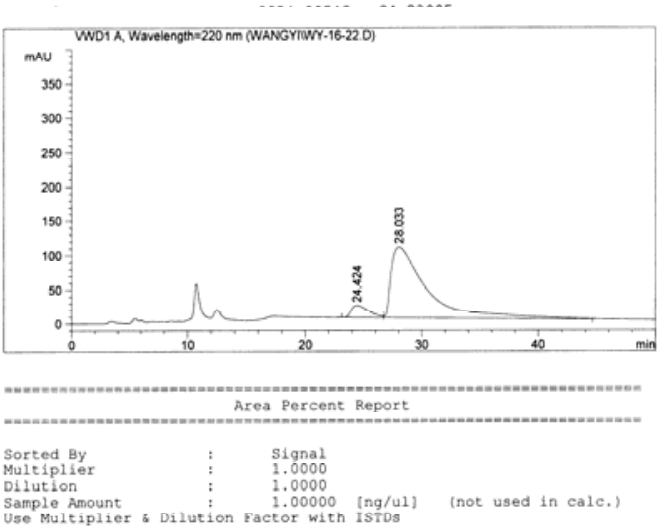

Signa1 1: VWD1 $A$, wavelength $=220 \mathrm{~nm}$

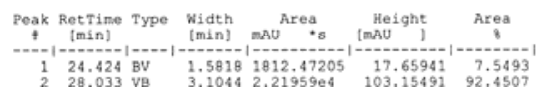

rotals : $\quad 2.40084 \mathrm{e} 4 \quad 120.81432$ 

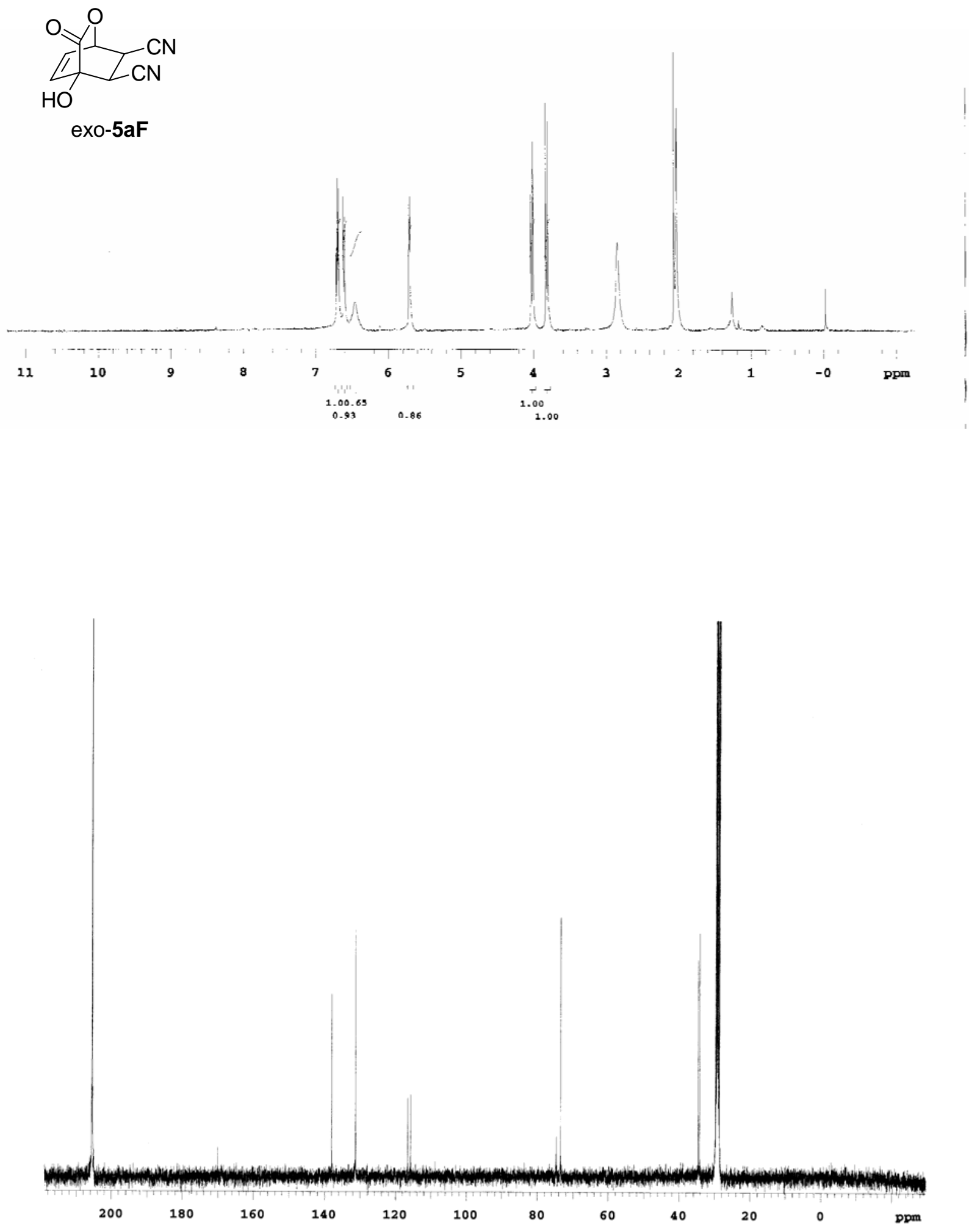

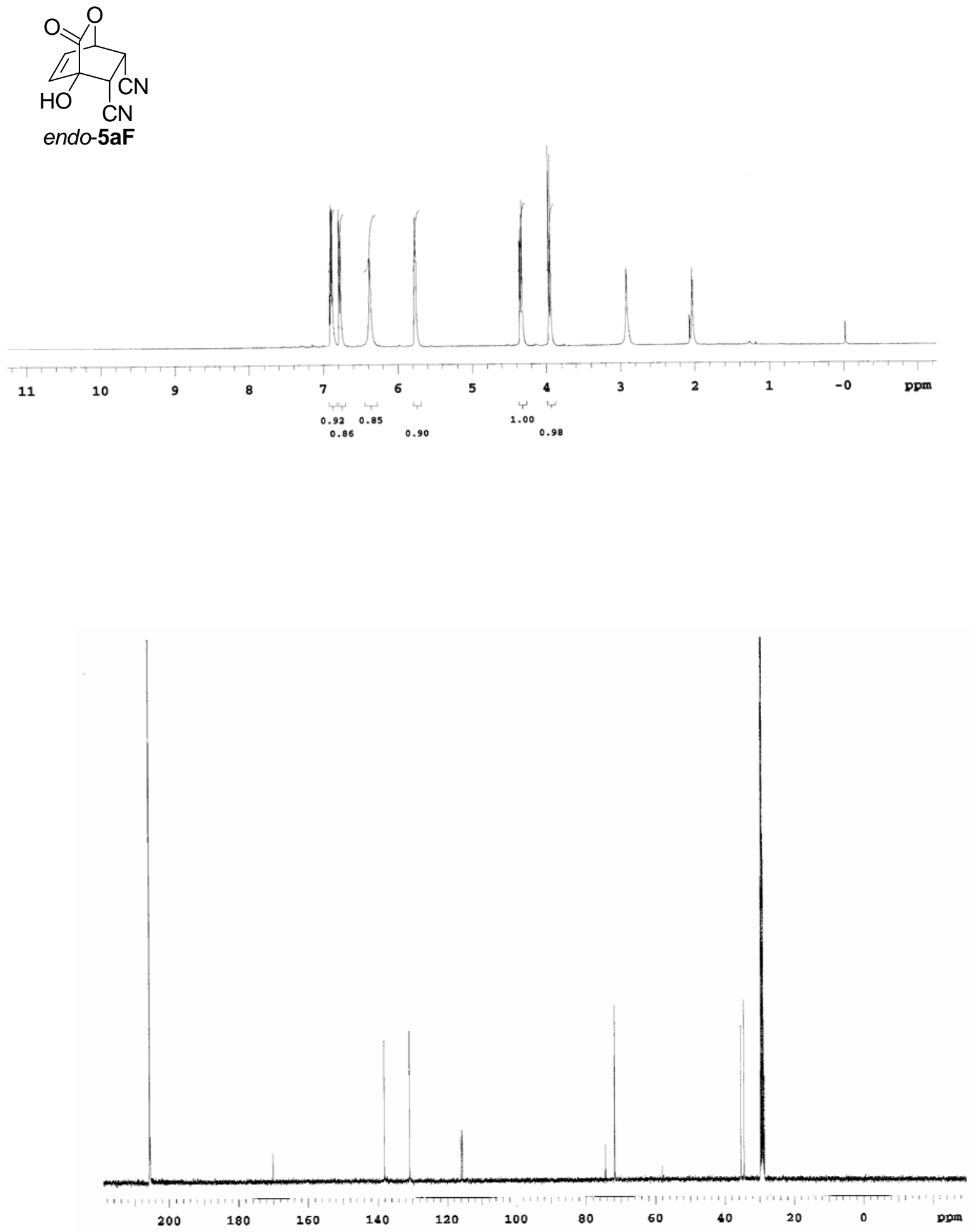
HPLC, Chiralpak AS, hexanes: isopropanol $=50: 50,1.0 \mathrm{~mL} / \mathrm{min}, \lambda=220 \mathrm{~nm}$
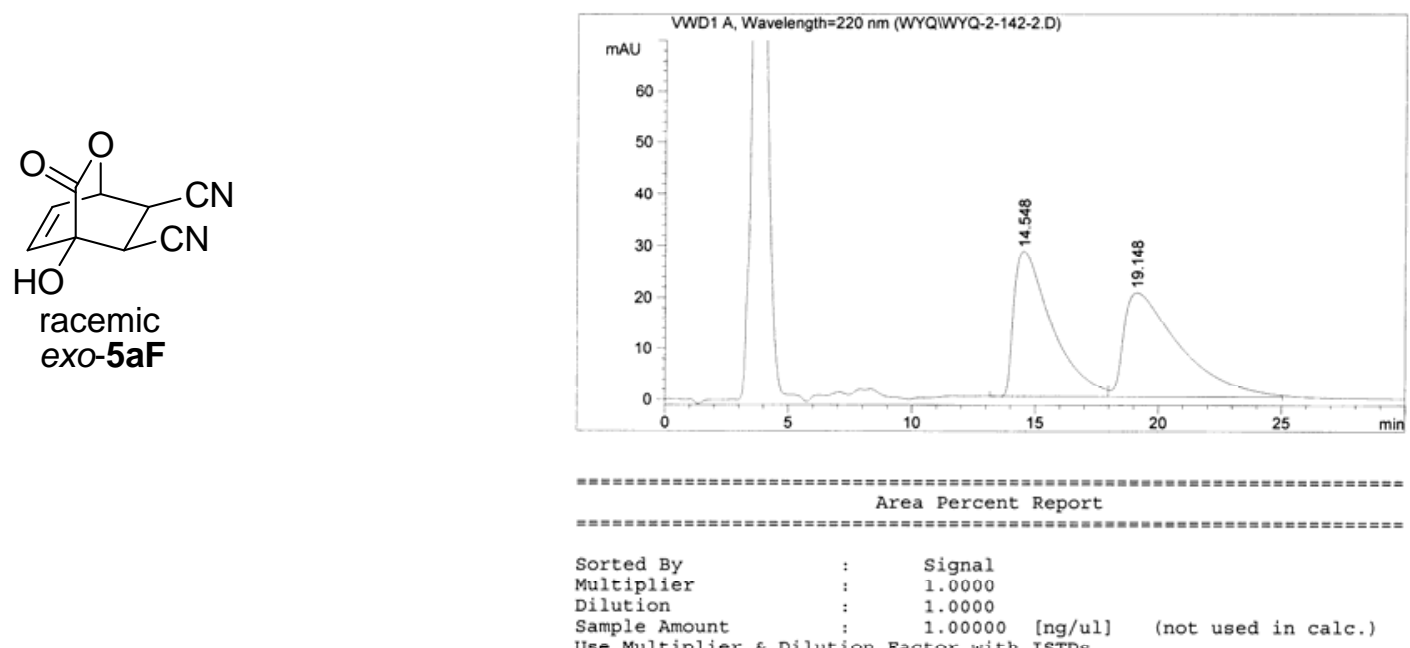

Use Multiplier \& Dilution Factor with 1 istos

Signal 1: VWD1 A, Wavelength=220 nm

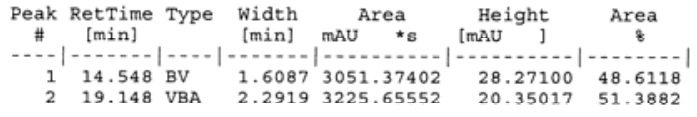

Totals : $\quad 6277.02954 \quad 48.62116$

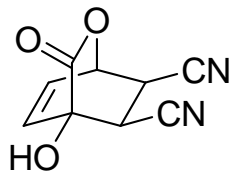

exo-5aF, 97\% ee obtained from QD-2 catalyzed reaction

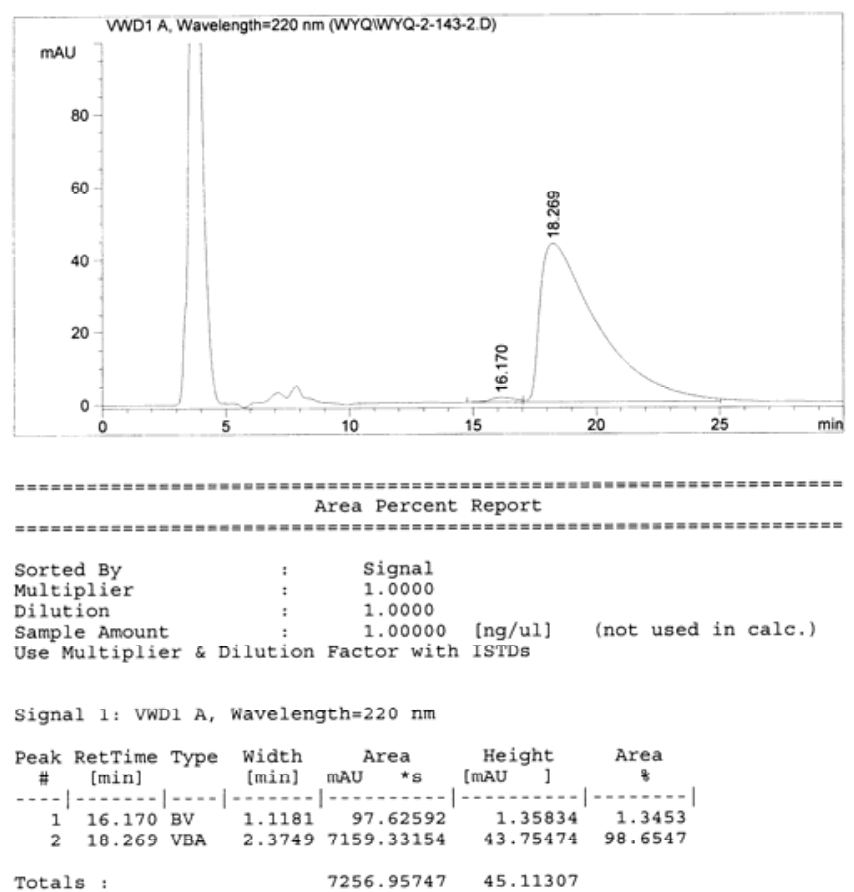


HPLC, Chiralpak AS, hexanes: isopropanol $=50: 50,1.0 \mathrm{~mL} / \mathrm{min}, \lambda=220 \mathrm{~nm}$

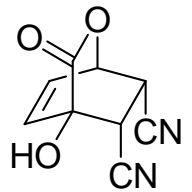

racemic endo-5aF
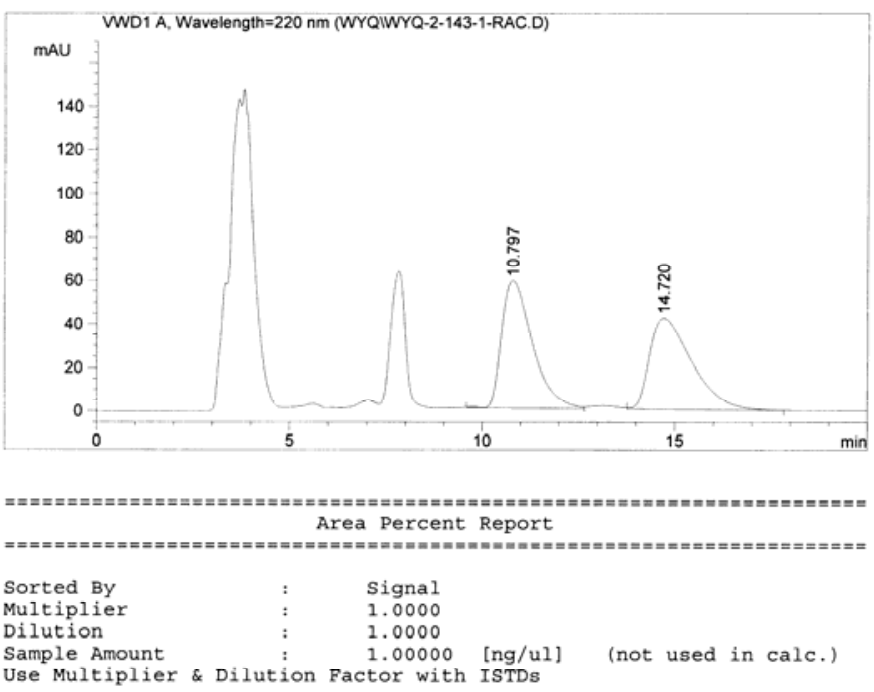

Signal 1: VWD1 $A$, wavelength $=220 \mathrm{~nm}$

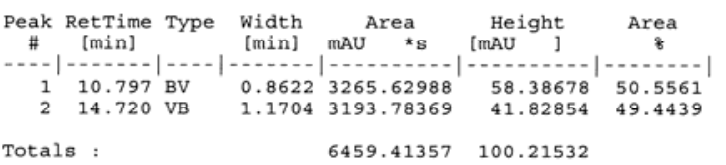

Totals :

$6459.41357 \quad 100.21532$
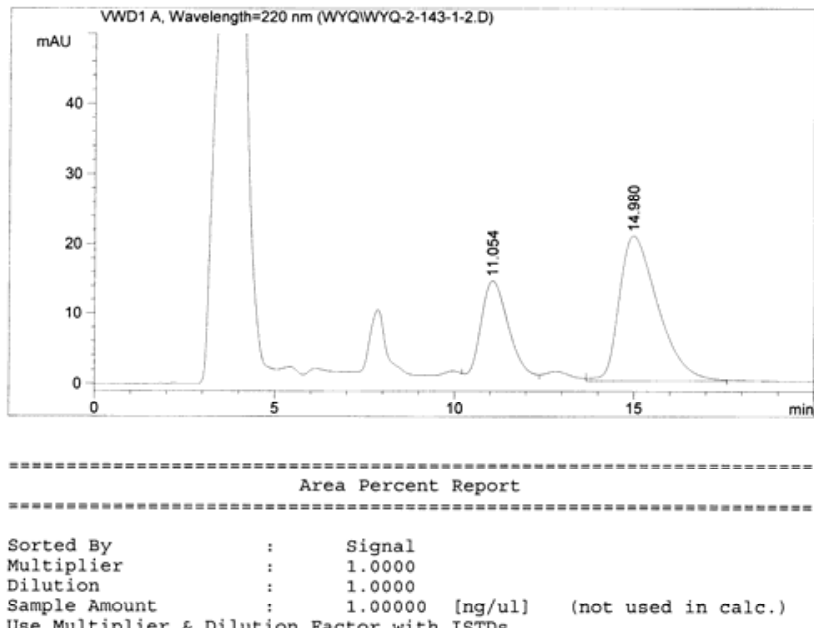

Use Multiplier \& Dilution Factor with ISTDs

Signal 1: VWD1 A, Wavelength $=220 \mathrm{~nm}$

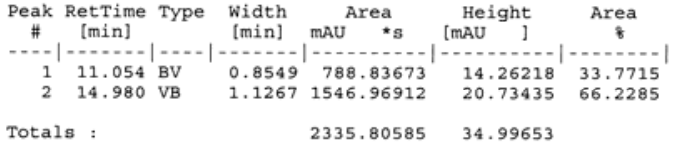


HPLC, Chiralpak AS, hexanes: isopropanol $=60: 40,1.0 \mathrm{~mL} / \mathrm{min}, \lambda=220 \mathrm{~nm}$
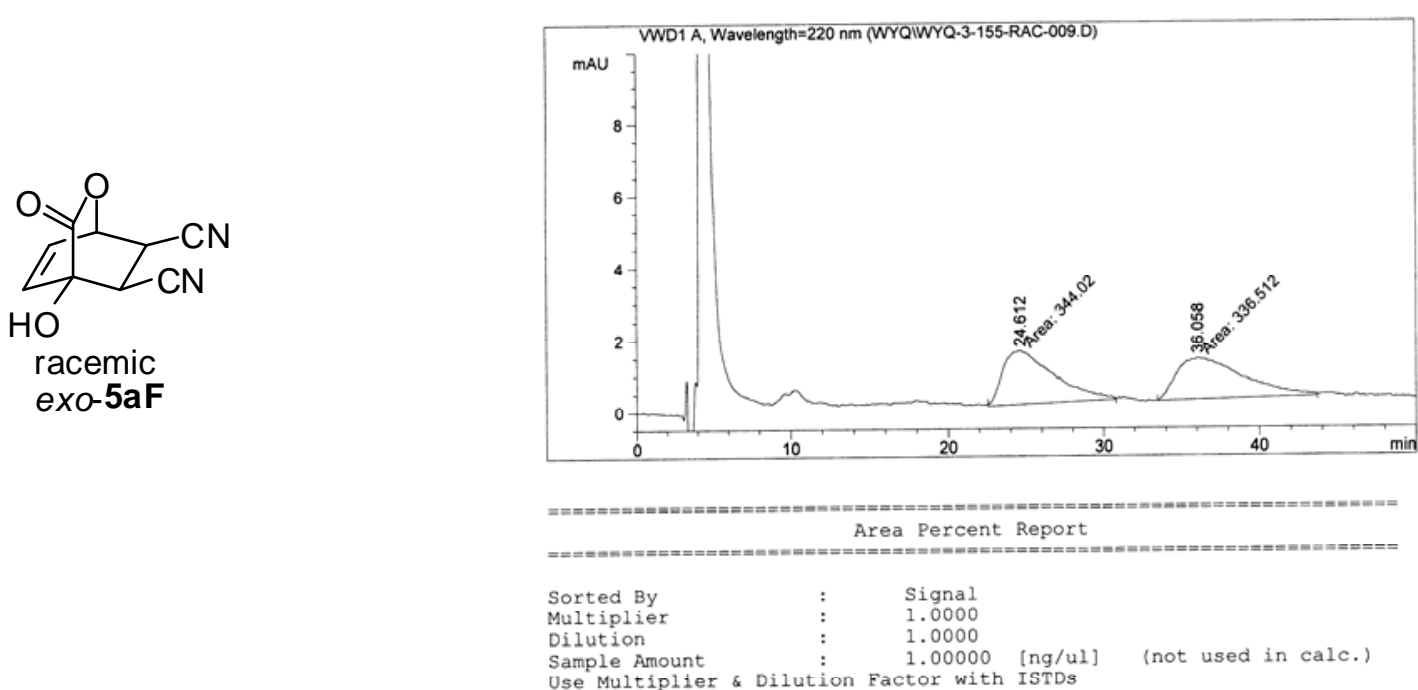

Use Multiplier \& Dilution Factor with ISTD

Signal 1: VWD1 $A$, wavelength $=220 \mathrm{~nm}$

\begin{tabular}{|c|c|c|c|c|c|c|}
\hline $\begin{array}{c}\text { Peak } \\
\#\end{array}$ & $\begin{array}{l}\text { RetTime } \\
{[\text { min] }}\end{array}$ & Type & $\begin{array}{l}\text { Width } \\
\text { [min] }\end{array}$ & ${ }_{\text {mAU }}{ }^{\text {Area }}{ }^{\circ}$ & $\begin{array}{r}\text { Height } \\
\text { [MAU ] }\end{array}$ & $\begin{array}{c}\text { Area } \\
8\end{array}$ \\
\hline $\begin{array}{l}1 \\
2\end{array}$ & $\begin{array}{l}24.612 \\
36.058\end{array}$ & $\begin{array}{l}\text { MM } \\
\text { MM }\end{array}$ & $\begin{array}{l}3.8331 \\
4.8905\end{array}$ & $\begin{array}{l}344.01965 \\
336.51227\end{array}$ & & $\begin{array}{l}50.5516 \\
49.4484\end{array}$ \\
\hline de & & & & 680.53192 & 2.64267 & \\
\hline
\end{tabular}

Totals :

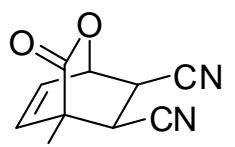

$\mathrm{HO}$

ent-exo-5aF, $>98 \%$ ee obtained from Q-2 catalyzed reaction
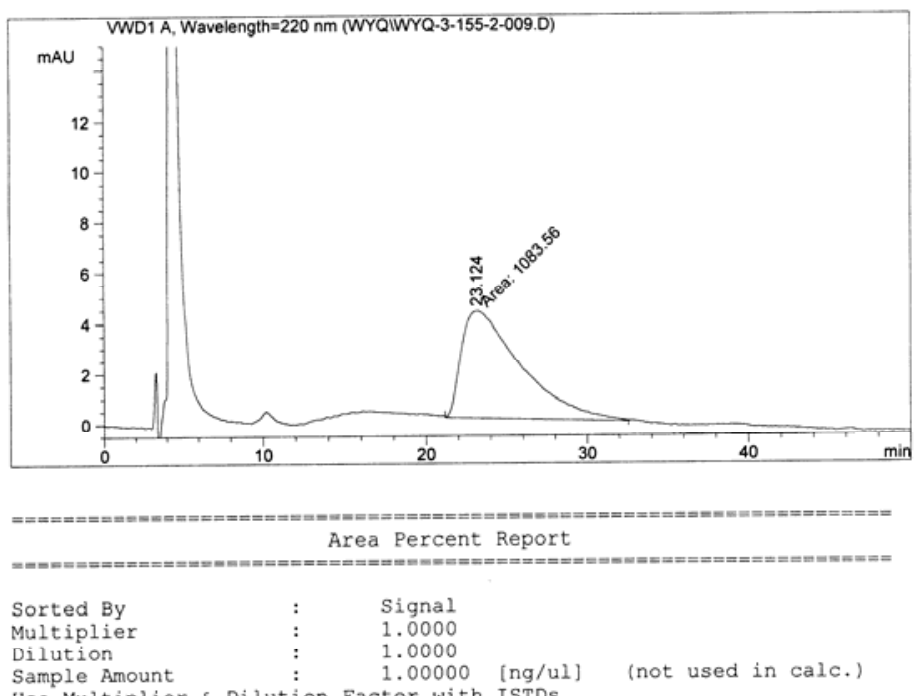

Use Multiplier \& Dilution Factor with ISTDS

Signal 1: VWD1 A, Wavelengt $h=220 \mathrm{~nm}$

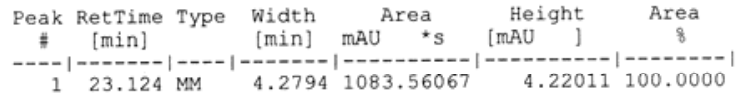

Totals : $\quad 1083.56067 \quad 4.22011$ 
HPLC, Chiralpak AS, hexanes: isopropanol $=60: 40,1.0 \mathrm{~mL} / \mathrm{min}, \lambda=220 \mathrm{~nm}$
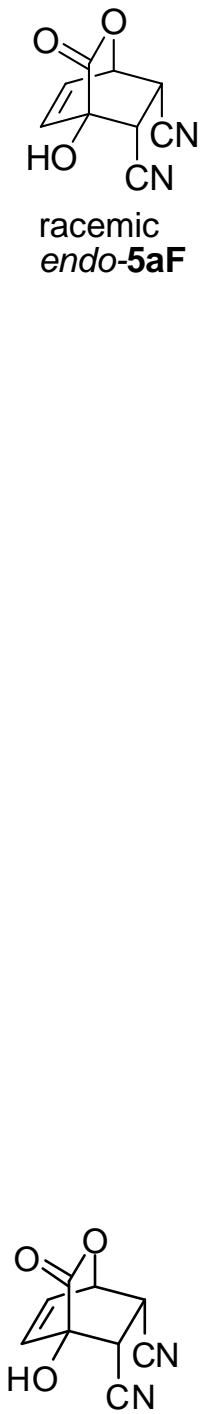

ent-endo-5aF, 37\% ee

obtained from Q-2

catalyzed reaction
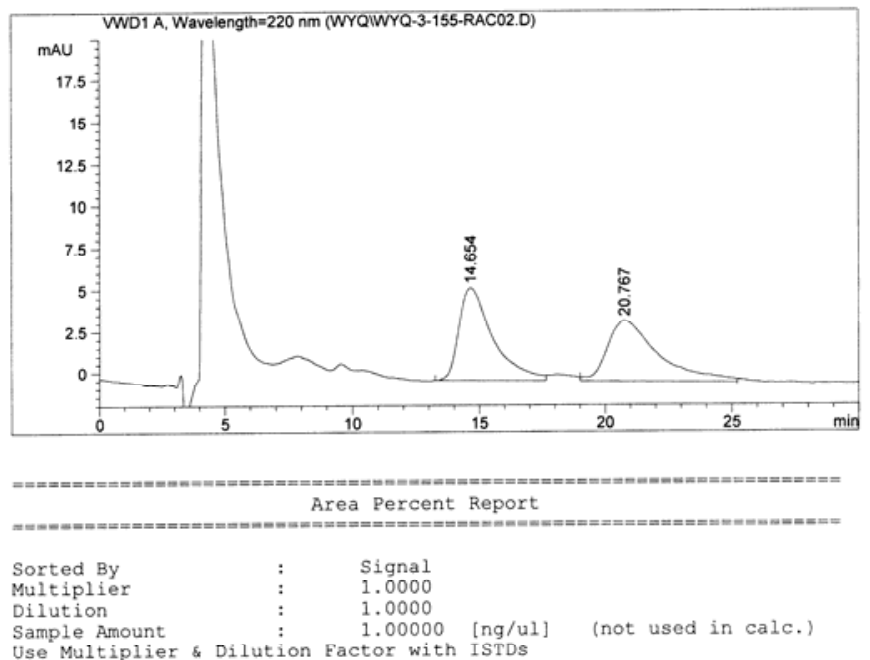

Signal 1: VWD1 $\mathrm{A}$, Wavelength=220 nm
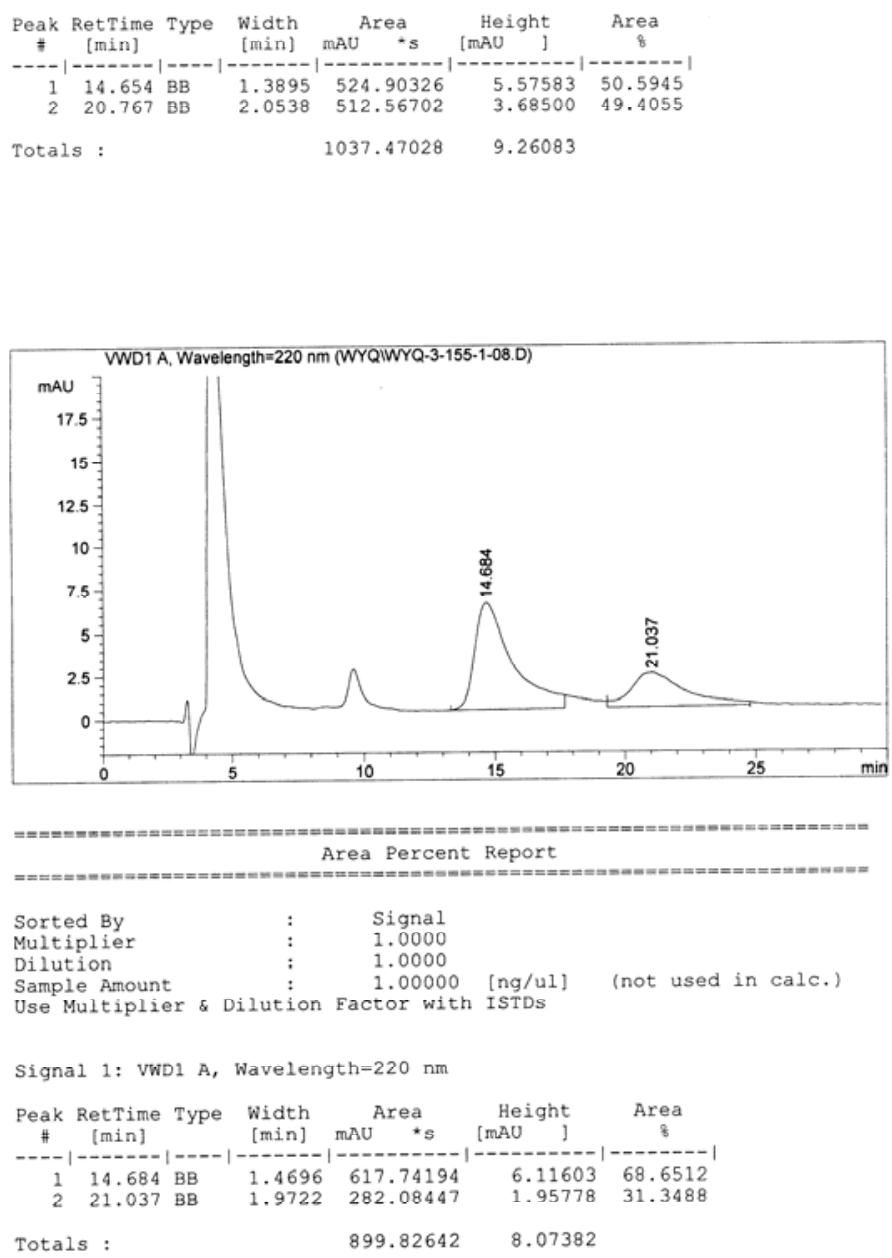

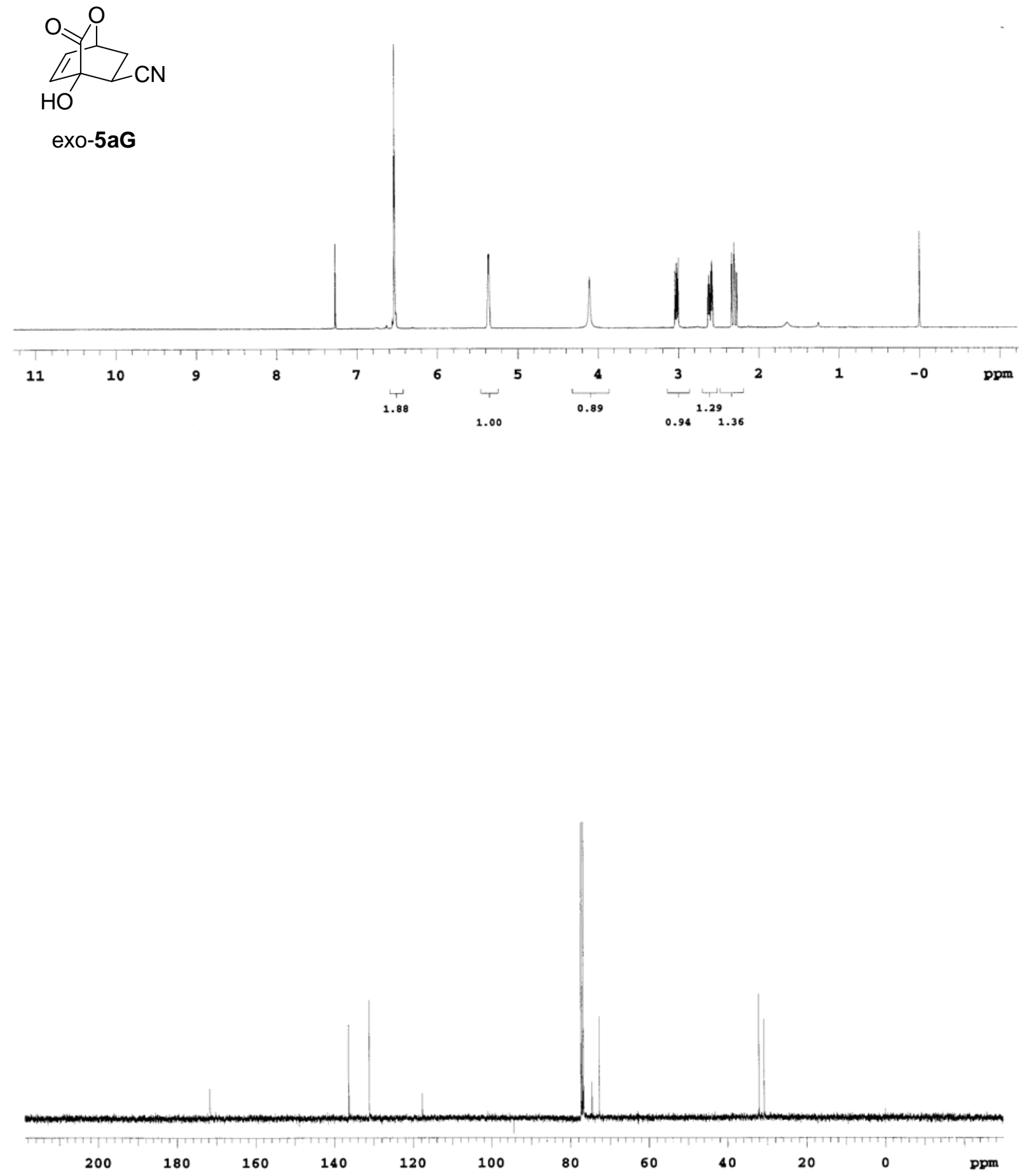
HPLC, Chiralcel OJ, hexanes: isopropanol $=60: 40,0.85 \mathrm{~mL} / \mathrm{min}, \lambda=220 \mathrm{~nm}$

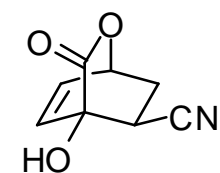

racemic 5 aG

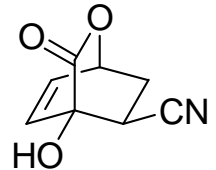

exo-5aG, 94\% ee obtained from QD-2 catalyzed reaction

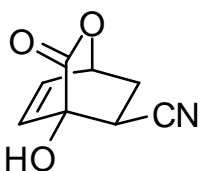

ent-exo-5aG, 93\% ee obtained from Q-2 catalyzed reaction
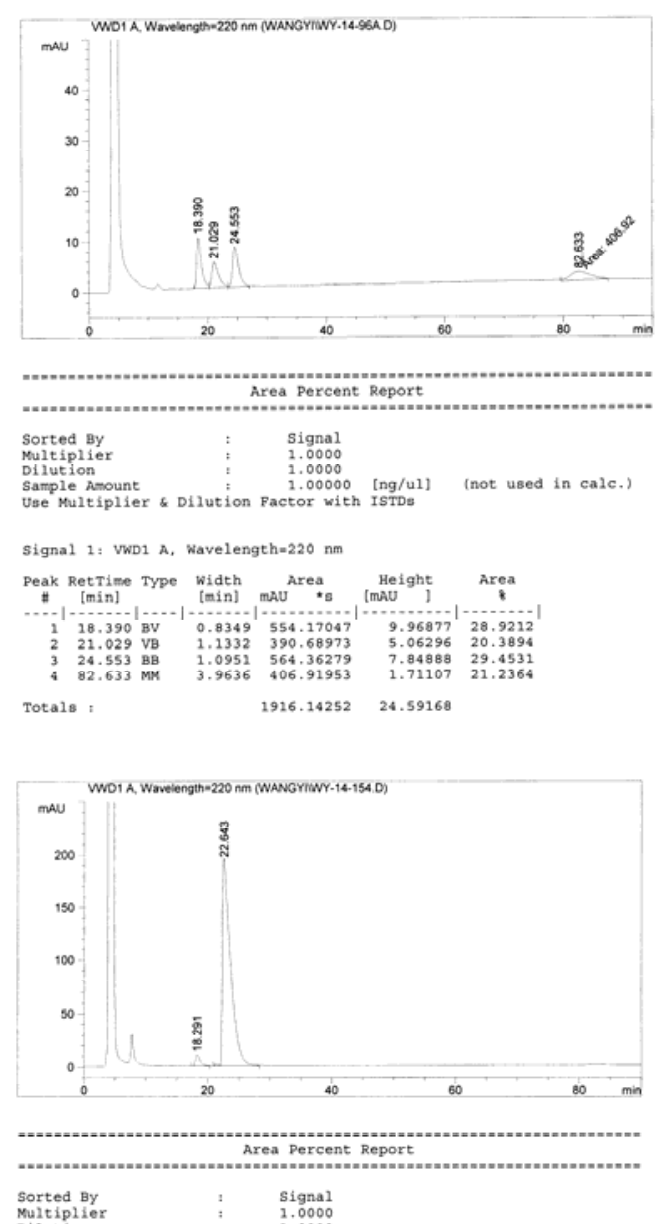

\begin{tabular}{l|l} 
Multiplier & 1.0000 \\
Dilution & 1.0000 \\
Sateplo & 1.00000
\end{tabular}

Dilution
Sasple Amount
Use Multiplier \& Dilution Pactor with 1.000000
ISTDs

Signal 1: VWD1 A, Wavelength=220 nm
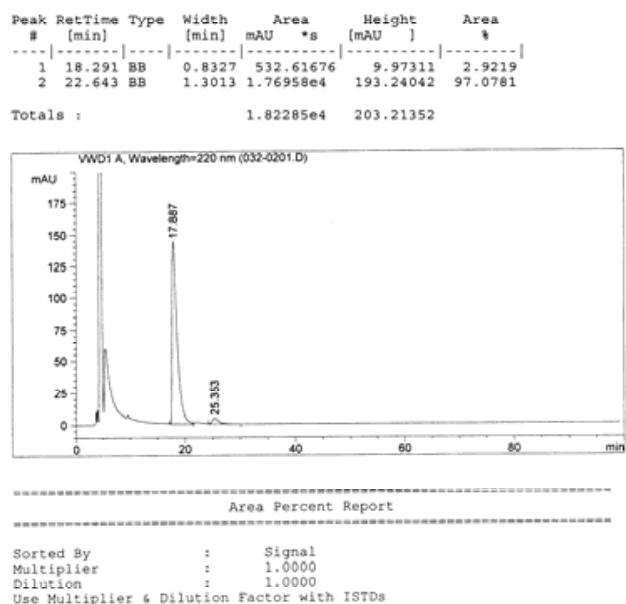

Dilution
Use Multiplier 6 Dilution Factor with ISTD

Signa1 1: VKD1 A., Wavelength=220 nim

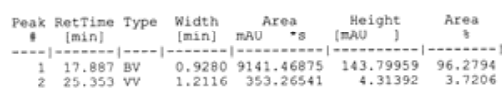

Totals : $\quad 9494.73416 \quad 148.11351$ 

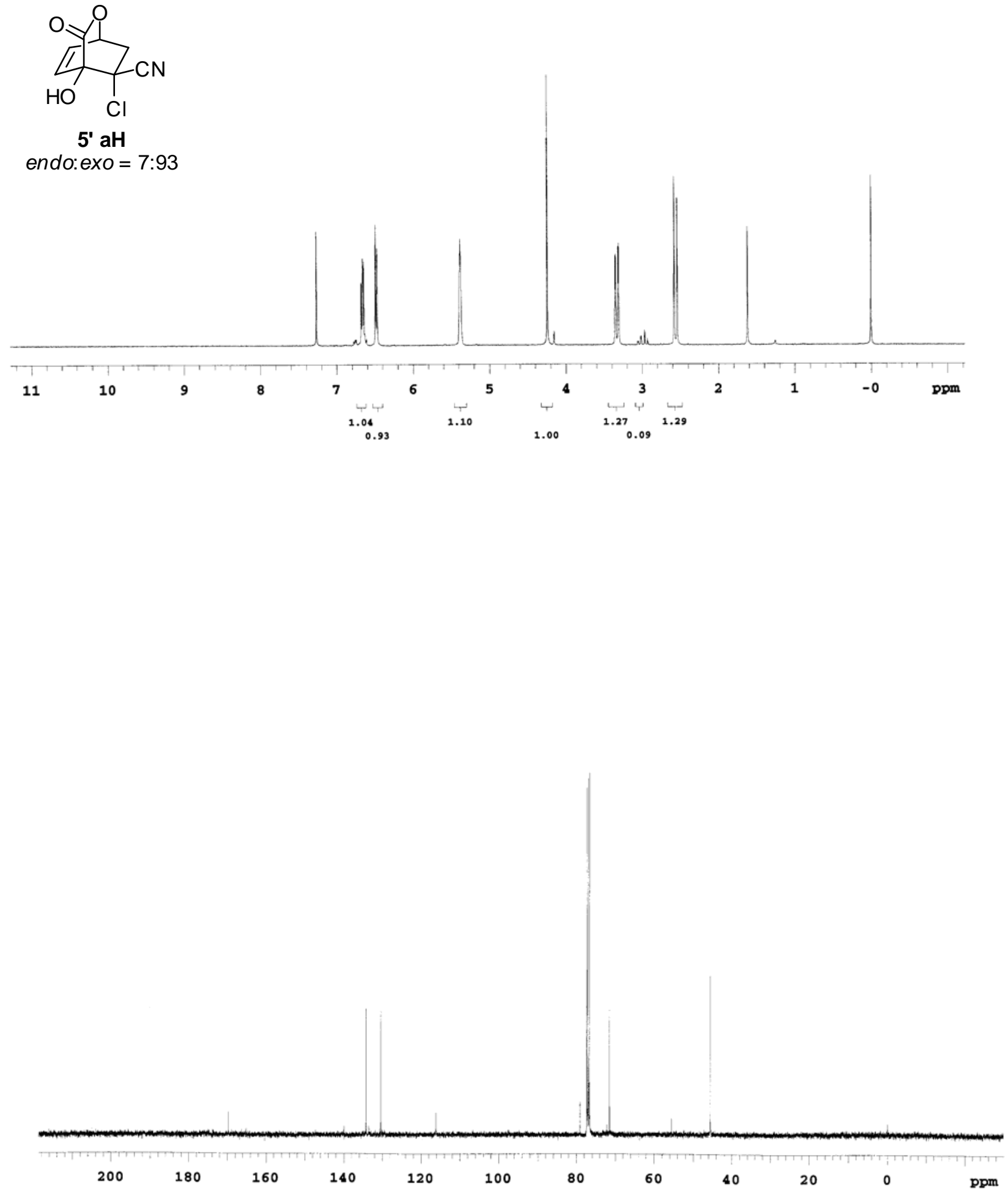

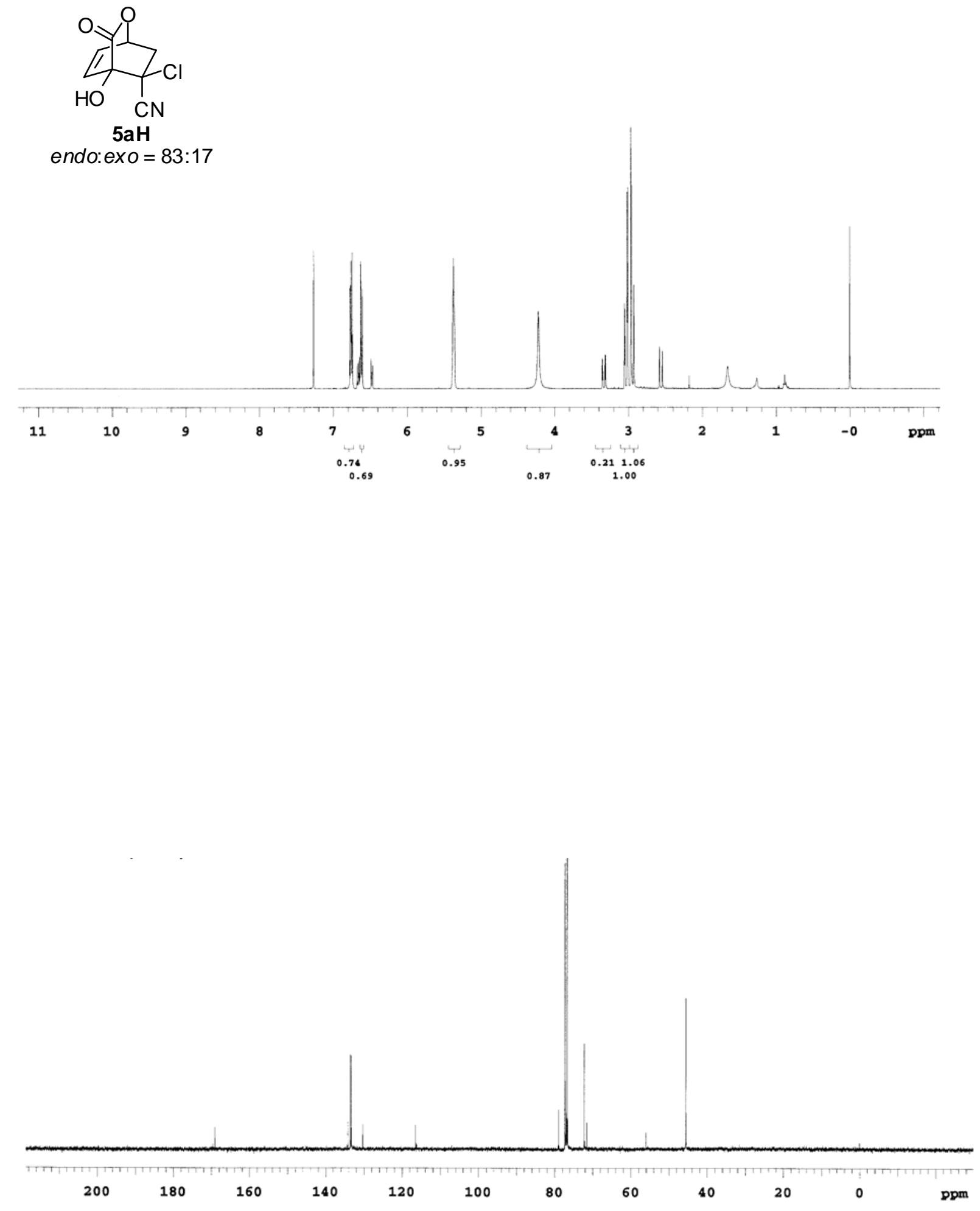
HPLC, AS, hexanes: isopropanol $=60: 40,0.4 \mathrm{~mL} / \mathrm{min}, \lambda=220 \mathrm{~nm}$.

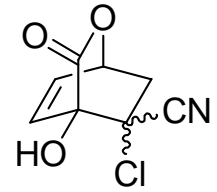

racemic 5 aH and 5' aH
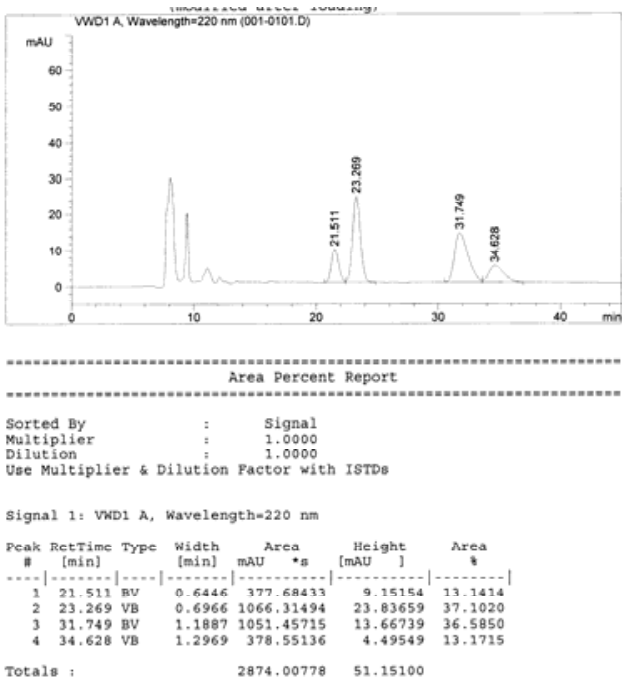

totals

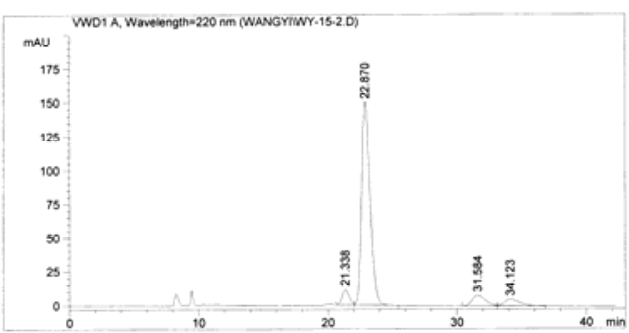

5aH $85 \%$ ee endo:exo = 83:17 obtained from QD-1a catalyzed reaction

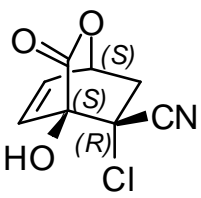

5'aH 89\%ee endo:exo = 7:93 obtained from QD-2 catalyzed reaction
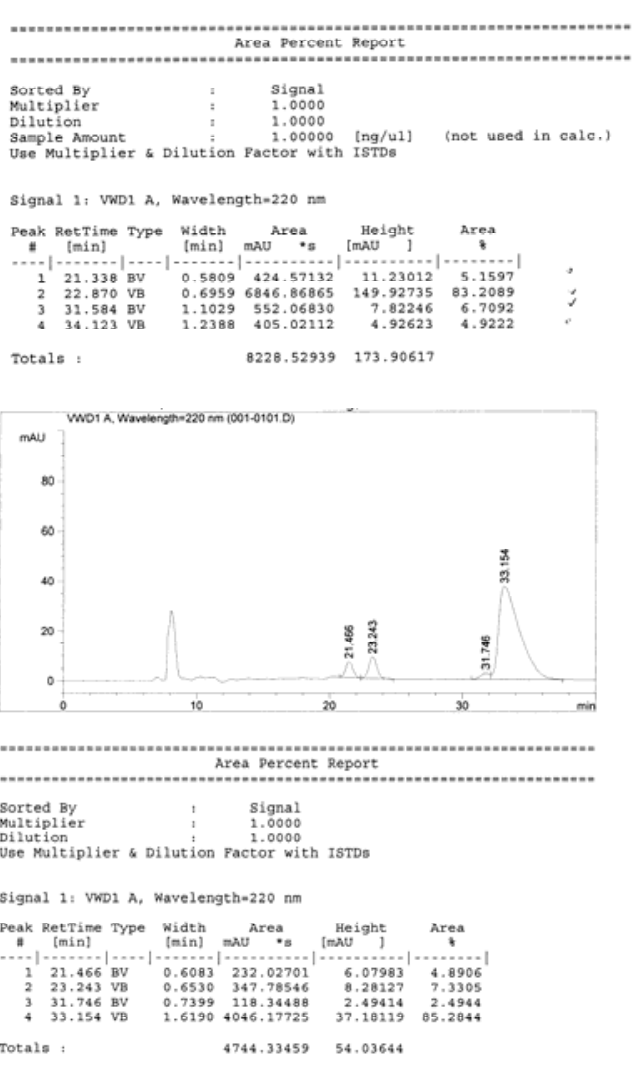
HPLC, AS, hexanes: isopropanol $=60: 40,0.4 \mathrm{~mL} / \mathrm{min}, \lambda=220 \mathrm{~nm}$.<smiles>N#C[C@]1(Cl)C2C=CC(O)(OC2)C1O</smiles>

racemic 5 aH and 5' aH

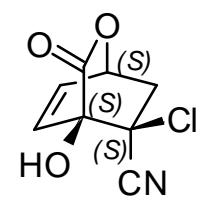

ent-5aH $75 \%$ ee endo:exo $=78: 22$ obtained from Q-1a catalyzed reaction

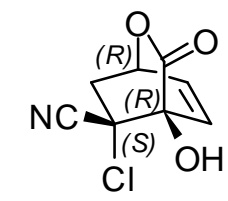

ent-5'aH 86\%ee endo:exo = 9:91 obtained from Q-2 catalyzed reaction
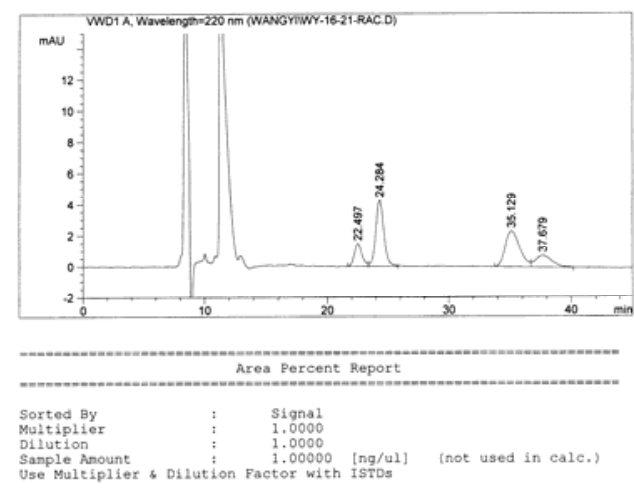

Signal 1: vaD1 $A$, wavelength $220 \mathrm{~nm}$
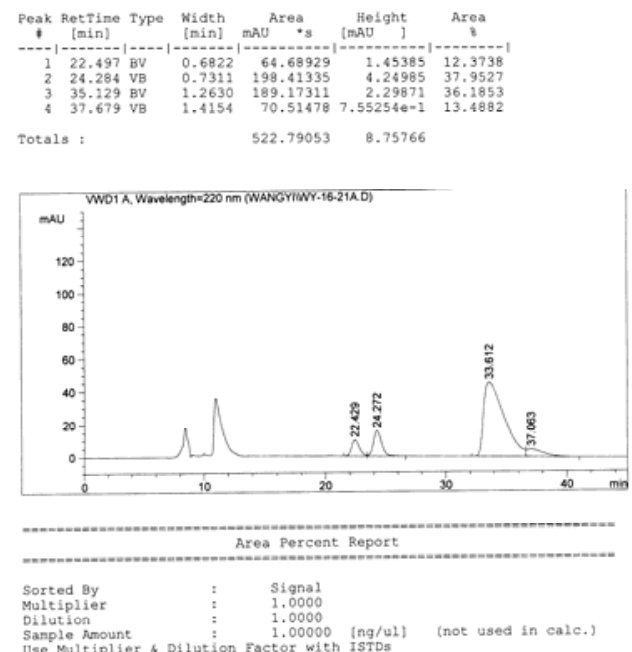

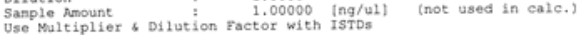

Signal 1: WWD1 A, wavelength=220 nm

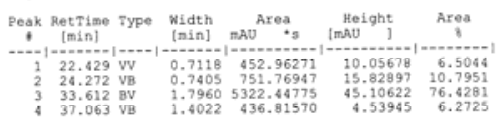

Totals : $\quad 6963.99564 \quad 75.53142$
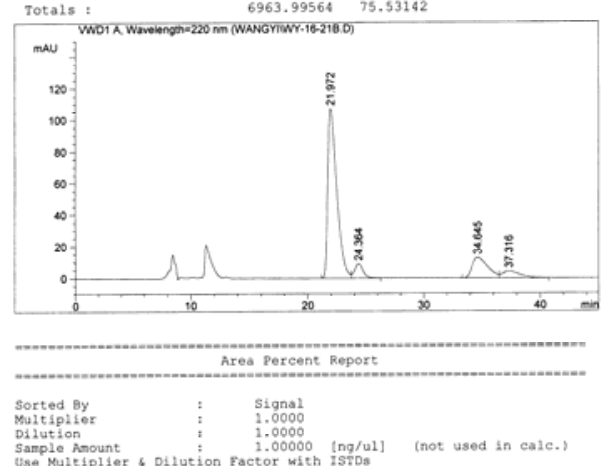

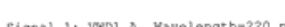

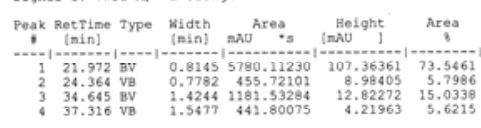

Totals : $\quad 7859.16690 \quad 133.39001$ 